\title{
Deterministic and Stochastic Dynamics in Spinodal Decomposition of a Binary System
}

\author{
D. O. Kharchenko, P. K. Galenko*,***, and V. G. Lebedev ${ }^{* * *}$ \\ Institute of Applied Physics, N.A.S. of the Ukraine, \\ 58 Petropavlivs'ka Str. \\ 40030 Sumy, Ukraine \\ "Institut für Materialphysik im Weltraum, \\ Deutsches Zentrum für Luft- und Raumfahrt (DLR), \\ 51170 Köln, Germany \\ **anstitut für Festkörperphysik, Ruhr-Universität Bochum, \\ 44780 Bochum, Germany \\ *:Udmurt State University, Department of Theoretical Physics, \\ 426034 Izhevsk, Russia
}

A model for diffusion and phase separation, which takes into account hyperbolic relaxation of the solute diffusion flux, is developed. Such a 'hyperbolic model' provides analysis of 'hyperbolic evolution' of patterns in spinodal decomposition in systems supercooled below critical temperature. Analytical results for the hyperbolic model of spinodal decomposition are summarized in comparison with outcomes of classic Cahn-Hilliard theory. Numeric modelling shows that the hyperbolic evolution leads to sharper boundary between two structures of a decomposed system in comparison with prediction of parabolic equation given by the theory of Cahn and Hilliard. Considering phase separation processes in stochastic systems with a field-dependent mobility and an internal multiplicative noise, we study dynamics of spinodal decomposition for parabolic and hyperbolic models separately. It is that the domain growth law is generalized when internal fluctuations are introduced into the model. A mean field approach is carried out in order to obtain the stationary probability, bifurcation and phase diagrams displaying re-entrant phase transitions. We relate our approach to entropy-driven phase-transitions theory.

Розвинуто модель дифузії та фазового розшарування, який враховує гіперболічну релаксацію дифузійного потоку. Такий «гіперболічний модель» призводить до «гіперболічного» рівнання щодо формування модульованих структур при спинодальнім розпаді в системах, охолоджених нижче критичної температури. Аналітичні результати для гіперболічного моделю спинодального розпаду порівнюються із відповідними резуль- 
татами, що випливають з класичної теорії Кана-Хіллярда. За допомогою чисельного моделювання показано, що еволюція системи в гіперболічнім моделю призводить до різкої міжфазної межі у порівнянні з обчисленнями за параболічним модельом Кана-Хіллярда. 3 розглядом процесів фазового розшарування в стохастичних системах із залежною від поля концентрації рухливістю та внутрішнім мультиплікативним шумом вивчається динаміка спинодального розпаду для параболічного та гіперболічного моделів. Показано, що закон зростання розмірів зерен може бути узагальнений введенням у розгляд внутрішніх флюктуацій, залежних від поля концентрації. Для дослідження стаціонарної картини (функції розподілу, біфуркаційних та фазових діяграм) розвинуто теорію середнього поля, в рамках якої встановлено, що відповідні перетворення носять реверсивний характер. Показано, що опис процесу фазового розшарування у стохастичних системах із внутрішнім шумом забезпечується використанням теорії ентропійнокерованих фазових переходів.

В работе развита модель для описания диффузии и фазового расслоения, которая учитывает гиперболическую релаксацию диффузионного потока. Такая «гиперболическая модель» приводит к гиперболическому уравнению описания формирования модулированных структур при спинодальном распаде в системах, охлажденных ниже критической температуры. Аналитические результаты для гиперболической модели спинодального распада сравниваются с соответствующими результатами, следующими из классической теории Кана-Хилларда. С помощью численного моделирования показано, что эволюция системы в гиперболической модели приводит к резким межфазным границам в сравнении с вычислениями согласно параболической модели Кана-Хилларда. При рассмотрении процессов фазового расслоения в стохастических системах с зависимой от поля концентрации подвижностью и внутренним мультипликативным шумом изучена динамика спинодального распада для параболической и гиперболической моделей. Показано, что закон роста размеров зерен может быть обобщен введением в рассмотрение внутренних флуктуаций, зависимых от поля концентрации. Для исследования стационарной картины (функции распределения, бифуркационных и фазовых диаграмм) развита теория среднего поля, в рамках которой установлено, что соответствующие превращения носят реверсивный характер. Показано, что описание процесса фазового расслоения в стохастических системах с внутренним шумом обеспечивается использованием теории энтропийноуправляемых фазовых переходов.

Key words: spinodal, diffusion, relaxation, model, liquid, structure factor, stochastic systems.

(Received March 6, 2009; in final version March 26, 2009)

\section{CONTENTS}

1. Introduction

2. Hyperbolic model for spinodal decomposition 
2.1. Hyperbolic transport

2.1.1. Equilibrium fluctuations

2.1.2. Power spectra of density and flux fluctuations

2.2. Hyperbolic spinodal decomposition

2.3. Dispersion relation and speeds for atomic diffusion

2.4. Critical parameters for hyperbolic decomposition

2.4.1. Critical wavelength for decomposition

2.4.2. Amplification rate of decomposition

2.4.3. Critical time for instability

2.4.4. Analysis of a structure function

2.5. Comparison with experimental data

3. Modelling of spinodal decomposition

3.1. $1 D$ modelling

3.2. $3 D$ modelling

4. Stochastic models of spinodal decomposition

4.1. Stochastic parabolic model for spinodal decomposition 4.1.1. An early stage of evolution

4.1.2. A late stage

4.1.3. Stationary case

4.1.4. Influence of external and internal noise sources

4.2. Stochasticity in hyperbolic transport

4.3. Stochastic hyperbolic model for spinodal decomposition 4.3.1. Early stages analysis

4.3.2. The effective Fokker-Planck equation for the hyperbolic model

5. Conclusions

Acknowledgments

References

\section{INTRODUCTION}

Consider a process of phase separation evolving through spontaneous growth of fluctuations, e.g., through fluctuations of concentration as in liquid-liquid systems or fluctuations of density as in gas-liquid systems. This process is known as a spinodal decomposition, in which, because of spontaneous fluctuations growth, both phases have equivalent symmetry but they differ only in composition. It was observed in many experiments on polymeric mixtures [1], liquid solutions [2, 3], organic systems [4], and metallic systems [5, 6]. This transformation has been widely investigated by using theoretical methods as well [7-10]. Phenomenological theory for decomposing phases has been constructed by Ginzburg and Landau [11]. They described magnetic domains in transition from the normal to superconducting phase using non-conserved order parameter. This theory has been successfully advanced by Cahn and Hilliard for using conserved order parameter for description of 
spinodal decomposition in binary liquids and solids [12]. As it has been further derived by Cahn [13, 14], kinetics of decomposition is defined by the growth of unstable fluctuations, and the mean size of a new phase can be given by the most rapidly growing fluctuation.

In parallel with detailed analysis $[10,15]$ and tests against experimental data [3, 4], the theory of Cahn and Hilliard has been further explored and developed. In particular, it has been demonstrated in computational modelling [16] that the rapidly quenched liquid mixtures under decomposition exhibit unusual non-equilibrium patterns, which do not consistent with predictions of the Cahn and Hilliard's theory. These inconsistencies might be associated with the phase segregation kinetics induced by hydrodynamic interactions following a rapid quench below spinodal [16]. They also might be attributed to the spinodal decomposition upon inhomogeneous quenching [17]. In both cases, there is a boundary for the critical quenching above which the classic Cahn and Hilliard's approach has to be extended to the case of strongly nonequilibrium decomposition provided by deep supercooling into the spinodal region of a phase diagram. Therefore, earliest stages and periods of decomposition under large supercooling can provide pattern's dynamics different from those predicted by the Cahn and Hilliard's theory.

A few advancements were made for strongly non-equilibrium phase separation. Binder, Frish, and Jäckle [18] generalized the linearized Cahn-Hilliard's theory to the case of existence of a slowly relaxing variable. Their calculations showed that the instability of the system is not of the standard diffusive type, but rather it is controlled by the relaxation of the slow structural variable. Recently, a hyperbolic diffusion equation with phase separation was derived in Refs [19, 20] from the formalism of extended irreversible thermodynamics [21]. It has been proposed that the hyperbolic equation is able to describe process of rapidly quenched decomposition for short periods of time, large composition gradients or deep supercoolings within a system. Finally, Grasselli et al. [22] mathematically analyzed extended Cahn-Hilliard's equation with hyperbolic relaxation of the diffusion flux. Their treatments have been devoted to one-, two-, and three-dimensional cases of hyperbolic spinodal decomposition [23-25] to establish existence of the global and exponential attractors for different phase spaces. These investigations [18-20,22-25] show that evolution of phase separation in deeply supercooled or rapidly quenched systems might be analyzed using predictions of hyperbolic transport equation.

It is known that considering the phase separation processes one need to take into account corresponding fluctuations, which lead to memory effects in the system dynamics. Memory effects in generalized transport equations play a relevant role at high frequency or high speed of perturbations. The influence of the non-vanishing relaxation time of 
the diffusion flux on the propagation of fast crystallization fronts has been studied [26, 27] in consistency with extended thermodynamics [21]. The memory effects play an important role in the propagation of phase interfaces during fast phase transitions [20].

Fluctuations for slow (i.e. internal energy, solute density etc.) and fast variables (e.g., heat flux and atomic diffusion flux) have been considered frequently. The fluctuations of the heat flux and the viscous pressure were stressed for the first time by Landau and Lifshitz [28], who derived the expressions for their correlation. In Refs [29, 30], the role of rapid fluctuations of the heat flux as a stochastic source has been considered within the extended thermodynamic formalism. In these works, a unified description of slow and fast heat fluctuations has been made [31] for equilibrium and non-equilibrium steady states. The same idea about separation of slow and fast variables to study fluctuations in a system of particles with inertia has been realized within the supersymmetric path-integral representation [32]. Besides density fluctuations, we explore the fluctuations of the diffusion flux and investigate their role in two different kinds of descriptions: (i) when the diffusion flux behaves as an independent fluctuating variable; (ii) the fluctuating part of the flux behaves as a stochastic noise in the evolution equation for the density.

To study the above-mentioned spatiotemporal phenomena in stochastic analysis, several analytical methods can be used. A linear stability analysis allows us to set the stability of a homogeneous state with respect to small perturbations in systems with fluctuating sources [33]. A fundamental study of noise-induced phase transitions can be provided by means of dynamic renormalization group theory [34]. In analytical investigation of noise-induced phenomena, a mean field approach is widely exploited (see Refs [35-38]). Despite the fact that the linear stability analysis can be used for a wide class of systems, the renormalization group approach cannot be used directly for all models of stochastic dynamics. The mean field theory has several modifications for systems with non-conserved and conserved dynamics. Such approach can be extended to a large number of stochastic systems to give a qualitative prediction of noise induced ordering and disordering phase transitions.

The main idea of the present review is to synthesize the previous results on hyperbolic model of spinodal decomposition and to analyze its predictions in comparison with outcomes of the parabolic model of Cahn and Hilliard. Formally, this review can be divided in two parts: the first one is devoted to study the hyperbolic model in the deterministic case, where we compare it with parabolic model for phase separation; in the second part, we discuss properties of two above stochastic models. In Section 2, free energy functional leading to hyperbolic governing equation for diffusion and phase separation is analyzed. Using 
dispersion relation, the main propagative peculiarities, such as phase and group speeds, are presented. Critical wavelength and time for instability together with amplification rate for hyperbolic decomposition are derived. Peculiarities of evolution of patterns are analyzed in Section 3 by numerical solution of the hyperbolic and parabolic transport equations. Here, we discuss a method to define the structure factor and results obtained in comparison with the outcomes from the Cahn and Hilliard's theory. Section 4 deals with stochastic approaches related to study the phase separation in parabolic and hyperbolic models. Starting from a stochastic parabolic model with a concentration dependent mobility, we introduce internal fluctuations, obeying fluctuation dissipation relation with an intensity reduced to the bath temperature. We show that at late stages of the system evolution the domain size growth (Lifshitz-Slyozov) law can be generalized in this model. Studying the stationary case in the mean field approximation, we present results of re-entrant behaviour of the effective order parameter, when it takes nontrivial values inside a fixed interval of the system parameters, and prove analytical investigations by computer simulations. In order to discuss stochastic hyperbolic model, we start with hyperbolic transport investigations. After, we consider stochastic hyperbolic model for phase separation and compare results obtained for two above stochastic models. Finally, in Section 5, a summary for the results is proposed.

\section{HYPERBOLIC MODEL FOR SPINODAL DECOMPOSITION}

In this Section, we introduce the hyperbolic model for spinodal decomposition. Starting from the hyperbolic transport equation, we analyze equilibrium fluctuations in the system described by two commensurable variables such as solute concentration and diffusion flux and discuss spectral properties of these fluctuations (Subsection 2.1). In Subsection 2.2, we discuss the hyperbolic model for phase separation in the deterministic case. The detailed study of the model is presented in Subsections 2.3, where we obtain the dispersion relation, group and phase speed, and perform the corresponding analysis, in Subsection 2.4 we discuss critical parameters for the hyperbolic model and analyze the structure function behaviour.

\subsection{Hyperbolic Transport}

Let us consider an isothermal and isobaric binary system (both the temperature $T$ and the pressure $P$ are constants) consisting of atoms $A$ and $B$. Following assumptions of Cahn [13], the system is represented as an isotropic solid solution free from imperfections and with 
the molar volume independent of concentration of $A$ - and $B$-atoms.

The system under study is described by the particle balance equation:

$$
\frac{\partial c}{\partial t}=-\nabla \cdot \mathbf{J}
$$

where $c$ is the particle density of a solute in a binary system, $\mathbf{J}-$ the diffusion flux, and $t$-the time. The diffusion flux is assumed to be described by the Maxwell-Cattaneo relaxation equation [20, $21,26,27]$,

$$
\tau_{D} \frac{\partial \mathbf{J}}{\partial t}+\mathbf{J}=-D \nabla c
$$

where $\tau_{D}$ and $D$ are the relaxation time and diffusion constant, respectively. The relaxation term is negligible for steady states or low-frequency perturbations. It becomes dominant at high frequencies or fast speed of propagation.

Density Profiles. Combining Eqs (1) and (2), one gets the following equation of a hyperbolic type

$$
\tau_{D} \frac{\partial^{2} c}{\partial t^{2}}+\frac{\partial c}{\partial t}=D \nabla^{2} c
$$

Equation (3) predicts the propagation of the density profile with a sharp front moving with a finite speed $V_{D}$ inside the undisturbed system. To show this feature of hyperbolic transport, we find an analytical solution of Eq. (3) for the semi-infinite (one-dimensional) space by choosing the initial and boundary conditions in the form $c(t, 0)=c_{f}, \quad c(0, x)=c(t, x \rightarrow \infty)=c_{0}, \quad \partial c(0, x) / \partial t=0$ (where $x$ is a spatial coordinate).

Under these conditions, the solution is described by the following expressions [39, 40]:

behind the diffusion front, $0 \leq x<t V_{D}$,

$$
\begin{gathered}
c(t, x)=c_{0}+\left(c_{f}-c_{0}\right) \exp \left(-x / l_{a}\right)+\left(c_{f}-c_{0}\right)\left(x / l_{a}\right) \int_{t=x / V_{D}}^{t} f(t, x) \mathrm{d} t, \\
f(t, x)=\frac{\exp \left(-t / 2 \tau_{D}\right)}{\left(t^{2}-x^{2} / V_{D}^{2}\right)^{1 / 2}} I_{1}\left[\frac{\left(t^{2}-x^{2} / V_{D}^{2}\right)^{1 / 2}}{2 \tau_{D}}\right] ;
\end{gathered}
$$

at the diffusion front, $x=t V_{D}$,

$$
c(t, x)=c_{0}+\left(c_{f}-c_{0}\right) \exp \left(-x / l_{a}\right) \equiv c_{0}+\left(c_{f}-c_{0}\right) \exp \left(-t / 2 \tau_{D}\right) ;
$$

ahead of the diffusion front, $t V_{D}<x<\infty$, 


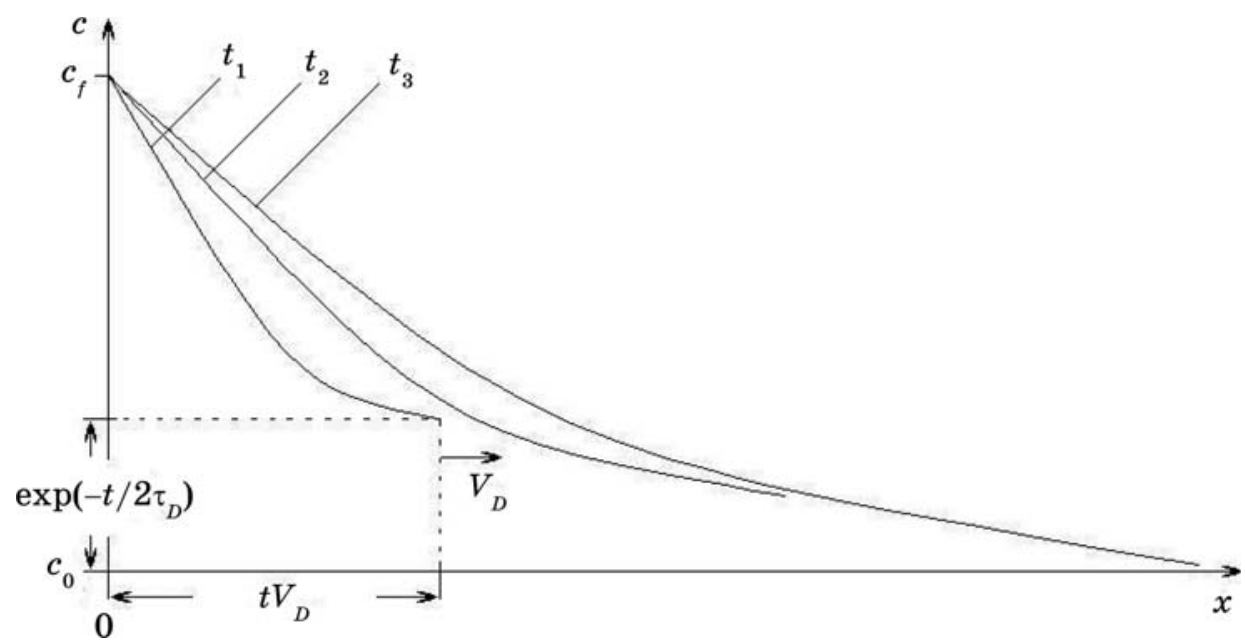

Fig. 1. Profiles of density $c$ at different moments $t_{1}<t_{2}<t_{3}$ as predicted by solution (4)-(6). Every profile moves with the sharp discontinuity front, which has the diffusion speed $V_{D}$. The $x$-coordinate of this discontinuity front is given by $t V_{D}$, and the amplitude of the front is decreasing in time as $\exp \left[-t /\left(2 \tau_{D}\right)\right]$. The density profile at $t=t_{3} \geq 10 \tau_{D}$ is matched to those one described by a partial differential equation of a parabolic type (i.e., of the form of Eq. (3) with $\tau_{D}=0$ ).

$$
c(t, x)=c_{0} .
$$

Here, $V_{D}=\left(D / \tau_{D}\right)^{1 / 2}$ and $l_{a}=2\left(D \tau_{D}\right)^{1 / 2}$ are the diffusion speed and the attenuation distance in the high-frequency limit [41], respectively, and $I_{1}$ is a modified Bessel function of the first order.

The concentration profiles described by Eqs (4)-(6) are shown in Fig. 1. In contrast with the concentration profiles described by the parabolic differential equation (Fick's diffusion), the concentration profiles in the hyperbolic case have a sharp diffusion front which moves with the speed $V_{D}$ (Fig. 1). This diffusion front separates the spatial regions where diffusion occurs ( $c>c_{0}$ at $x<V_{D} t$; Eq. (4)) and where diffusion is absent ( $c=c_{0}$ at $x>V_{D} t$; Eq. (6)). Therefore, the position of the diffusion front may be examined as a depth, $t V_{D}$, of density penetration into a binary system. As it is shown in Fig. 1, the amplitude of the diffusive front at $x=V_{D} t$ decreases with increasing time and spatial coordinate, according to Eq. (5).

\subsubsection{Equilibrium Fluctuations}

Even though solution (4)-(6) describes a smooth profile of density (with sharp diffusion front) fluctuations always exist in a thermody- 
namic system. Indeed, techniques of light scattering or neutron scattering allow to explore details of the dynamics of density perturbations in the system, and it has fostered progress in nonequilibrium statistical mechanics [42]. Following Ref. [40], we describe features of the system described by equations (1) and (2) related to $c$ and $J$ fluctuations in equilibrium and to stochastic noise.

The equilibrium second moments of fluctuations of $c$ and $\mathbf{J}$ are obtained from the Einstein's equation for the probability of fluctuations $[28,43]$, namely

$$
\operatorname{Pr} \propto \exp \left[\frac{\delta^{2} s}{2 k_{B} T}\right],
$$

where entropy $s(c, \mathbf{J})$ is based on the independent thermodynamic variables $c$ and $\mathbf{J}$. It is known (see, e.g., Ref. [43], Chapter 15) that Einstein's equation (7) considered as an approximate Gaussian distribution function predicts the second moments correctly, but it does not predict third and higher moments accurately. However, since we are only interested in the second moments, we restrict ourselves in this Section to the use of the simple Einstein formula (7).

To obtain the second differential $\delta^{2} s$ of entropy in Einstein's equation (7), one needs to choose the form of the Gibbs equation for entropy. The generalized Gibbs equation, which incorporates slow and fast thermodynamic variables, is written as [21]

$$
d s=\frac{1}{T} d u-\frac{\mu}{T} d c-\frac{\tau_{D}}{T \bar{D}} \mathbf{J} \cdot d \mathbf{J},
$$

where $u$ is a density of internal energy, $D$ is related to the usual diffusion coefficient $D$ through $D=\bar{D} \partial \mu / \partial c$, and $\mu=\mu_{1}-\mu_{2}$ is the relative chemical potential of the solute with respect to the one of the solvent.

We focus our attention on the fluctuations of $c$ and $\mathbf{J}$ and assume $d u$ negligible for the sake of simplicity. Then, from Eq. (8), we get the second differential of the entropy as

$$
\delta^{2} s=-\frac{1}{T} \frac{\partial \mu}{\partial c}(\delta c)^{2}-\frac{\tau_{D}}{T \bar{D}}(\delta J)^{2} .
$$

With the definition (7) and taking the second variation of $s$ from Eq. (9), the probability of fluctuations is described by

$$
\operatorname{Pr}(\delta c, \delta J) \propto \exp \left[-\frac{v}{2 k_{B} T}\left(\frac{\partial \mu}{\partial c}\right)(\delta c)^{2}-\frac{v \tau_{D}}{2 k_{B} T \bar{D}}(\delta J)^{2}\right],
$$

where $v$ is a small volume in which the fluctuations $\delta c$ and $\delta \mathbf{J}$ occur. The second moments of fluctuations are given by 


$$
\left\langle(\delta c)^{2}\right\rangle=\frac{k_{B} T}{v(\partial \mu / \partial c)_{T}}, \quad\left\langle(\delta J)^{2}\right\rangle=\frac{k_{B} T \bar{D}}{v \tau_{D}}=\frac{k_{B} T D}{v \tau_{D}(\partial \mu / \partial c)_{T}} .
$$

In what follows, we discuss two important points: (i) the power spectra of the fluctuations of $c$ and $\mathbf{J}$, (ii) the description of the stochastic sources in the system (1) and (2).

\subsubsection{Power Spectra of Density and Flux Fluctuations}

Let us define the correlation functions for the fluctuations of $c$ and $\mathbf{J}$ in the following usual form

$$
\begin{aligned}
C_{c}\left(\mathbf{r}, \mathbf{r}^{\prime}, t, t^{\prime}\right) & \equiv\left\langle\delta c(\mathbf{r}, t) \delta c\left(\mathbf{r}^{\prime}, t^{\prime}\right)\right\rangle, \\
C_{J}\left(\mathbf{r}, \mathbf{r}^{\prime}, t, t^{\prime}\right) & \equiv\left\langle\delta \mathrm{J}(\mathbf{r}, t) \delta \mathrm{J}\left(\mathbf{r}^{\prime}, t^{\prime}\right)\right\rangle,
\end{aligned}
$$

where $\mathbf{r}$ is the position vector of a point in the system. Since we consider equilibrium (homogeneous, time-invariant state), one has

$$
\begin{aligned}
& C_{c}\left(\mathbf{r}, \mathbf{r}^{\prime}, t, t^{\prime}\right)=C_{c}\left(\mathbf{r}-\mathbf{r}^{\prime}, t-t^{\prime}\right), \\
& C_{J}\left(\mathbf{r}, \mathbf{r}^{\prime}, t, t^{\prime}\right)=C_{J}\left(\mathbf{r}-\mathbf{r}^{\prime}, t-t^{\prime}\right),
\end{aligned}
$$

i.e. the correlation functions depend only on relative distances $\mathbf{r}-\mathbf{r}^{\prime}$ and on the difference in time $t-t^{\prime}$. We are interested in the Fourier transforms of the quantities in Eq. (13), namely

$$
\begin{aligned}
& S_{c}(\omega, \mathbf{k})=\int e^{i \omega t} e^{i \mathbf{k r}} C_{c}(\mathbf{r}, t) d \mathbf{r} d t, \\
& S_{J}(\omega, \mathbf{k})=\int e^{i \omega t} e^{i \mathbf{k r}} C_{J}(\mathbf{r}, t) d \mathbf{r} d t .
\end{aligned}
$$

These expressions represent fluctuation spectra and have special theoretical and practical interest, as they may be measured by means of light scattering or neutron scattering techniques [42].

To obtain an explicit form of the fluctuation spectra we first write Fourier transform (in space) and Laplace transform (in time) of equations (1) and (2). Using the standard procedure described in Refs [21, 42], we arrive at

$$
\begin{gathered}
S \delta c_{\mathbf{k}}(S)+i \mathbf{k} \delta J_{\mathbf{k}}(S)=\delta c_{\mathbf{k}}(0), \\
\tau_{D} S \delta J_{\mathbf{k}}(S)+\delta J_{\mathbf{k}}(S)+i \mathbf{k} D \delta c_{\mathbf{k}}(S)=\delta J_{\mathbf{k}}(0),
\end{gathered}
$$

where $\delta c_{\mathrm{k}}(S)$ and $\delta J_{\mathrm{k}}(S)$ are the Fourier-Laplace components of $\delta c$ and $\delta J$, respectively. Then, we have

$$
\left(\begin{array}{c}
\delta c_{\mathbf{k}}(S) \\
\delta J_{\mathbf{k}}(S)
\end{array}\right)=\frac{1}{S\left(1+\tau_{D} S\right)+D k^{2}}\left[\begin{array}{cc}
1+\tau_{D} S & -i \mathbf{k} D \\
-i \mathbf{k} & S
\end{array}\right]\left(\begin{array}{c}
\delta c_{\mathbf{k}}(0) \\
\delta J_{\mathbf{k}}(0)
\end{array}\right)
$$


In equilibrium state (where $|\mathbf{k}| \rightarrow 0$ ), the crossed second moments, $\left\langle\delta c_{\mathbf{k}}(0) \delta J_{\mathbf{k}}(0)\right\rangle$, vanish because they have opposite time-reversal parity.

Then, from Eq. (16), we have

$$
\begin{gathered}
\left\langle\delta c_{\mathrm{k}}(S) \delta c_{\mathrm{k}}(0)\right\rangle=\frac{1+\tau_{D} S}{S\left(1+\tau_{D} S\right)+D k^{2}}\left\langle\left|\delta c_{\mathrm{k}}(0)\right|^{2}\right\rangle, \\
\left\langle\delta J_{\mathrm{k}}(S) \delta J_{\mathrm{k}}(0)\right\rangle=\frac{S}{S\left(1+\tau_{D} S\right)+D k^{2}}\left\langle\left|\delta J_{\mathrm{k}}(0)\right|^{2}\right\rangle .
\end{gathered}
$$

To obtain the time Fourier transform, one may write

$$
\begin{aligned}
& S_{c}(\omega, k=\mathbf{k} \mid)=2 \Re\left[\left\langle\delta c_{\mathbf{k}}(S=i \omega) \delta c_{\mathbf{k}}(0)\right\rangle\right], \\
& S_{J}(\omega, k=|\mathbf{k}|)=2 \Re\left[\left\langle\delta J_{\mathbf{k}}(S=i \omega) \delta J_{\mathbf{k}}(0)\right\rangle\right] .
\end{aligned}
$$

Finally, we obtain

$$
\begin{aligned}
& S_{c}(\omega, k)=\frac{2 D k^{2}}{\tau_{D}^{2} \omega^{4}+\left(1-2 D \tau_{D} k^{2}\right) \omega^{2}+\left(D k^{2}\right)^{2}}\left\langle\left|\delta c_{\mathbf{k}}(0)\right|^{2}\right\rangle, \\
& S_{J}(\omega, k)=\frac{2 \omega^{2}}{\tau_{D}^{2} \omega^{4}+\left(1-2 D \tau_{D} k^{2}\right) \omega^{2}+\left(D k^{2}\right)^{2}}\left\langle\left|\delta J_{\mathbf{k}}(0)\right|^{2}\right\rangle .
\end{aligned}
$$

The corresponding expressions for $\left\langle\left|\delta c_{\mathrm{k}}(0)\right|^{2}\right\rangle$ and $\left\langle\left|\delta J_{\mathrm{k}}(0)\right|^{2}\right\rangle$ in equilibrium obtained from Eq. (11) are described by

$$
\left\langle\left|\delta c_{\mathbf{k}}(0)\right|^{2}\right\rangle=\frac{k_{B} T}{v(\partial \mu / \partial c)_{T}}, \quad\left\langle\left|\delta J_{\mathbf{k}}(0)\right|^{2}\right\rangle=\frac{2 k_{B} T}{v \tau_{D}(\partial \mu / \partial c)_{T}} .
$$

Note that, in Eq. (19), the function $S_{c}(\omega, k)$ has a maximum at a frequency $\omega_{m}$ given by

$$
\omega_{m}=\left[\left(2 D k^{2} \tau_{D}-1\right) /\left(2 \tau_{D}^{2}\right)\right]^{1 / 2} .
$$

The fact that the maximum is at $\omega_{m} \neq 0$ indicates propagation of density waves with the speed $k / \omega_{m}$, in contrast with the situation when the maximum is at $\omega_{m}=0$, which means purely diffusive transport. It is clear from Eq. (20) that, to observe such a maximum, i.e., the propagation of density wave, it is needed that $k>k_{c} \equiv\left(2 D \tau_{D}\right)^{-1 / 2}$.

Thus, for $k<k_{c}$, transport is diffusive, and for $k>k_{c}$, the density waves may propagate.

This analysis is analogous to the analysis of the transverse velocity correlation function in generalized thermodynamics for the Maxwell viscoelastic model [42], which is consistent with the formalism of extended irreversible thermodynamics [21]. 


\subsection{Hyperbolic Spinodal Decomposition}

Let us consider a region of phase diagram, in which one phase mixture is unstable with respect to decomposition. This is a spinodal region where curvature of free energy is negative:

$$
\frac{\partial^{2} f}{\partial c^{2}}<0
$$

and the spinodal itself is defined by

$$
\frac{\partial^{2} f}{\partial c^{2}}=0
$$

where $f$ is the Helmholtz free energy per unit volume and $c$ is the concentration of $B$ atoms.

For a given temperature, the free energy $f$ is based on the following variables: concentration $c$, gradient of concentration $\nabla c$, and solute diffusion flux J. Dependence of free energy on concentration is due to existence of a diffuse interface between appearing phases in which high concentration gradients may exist. Dependence of free energy on diffusion flux reflects of the fact that decomposition may proceed with high rates comparable with the speed $V_{D}=\left(D / \tau_{D}\right)^{1 / 2}$ of the front of solute diffusion profile, where $D$ is the diffusion coefficient and $\tau_{D}$ the time for relaxation of the solute diffusion flux to its steady-state value. Thus, the selected set of independent variables $\{c, \nabla c, \mathbf{J}\}$ consists of slow conserved variable $c$, fast non-conserved variable $\mathbf{J}$, and gradient variable $\nabla c$. Analogous set of variables is generally analyzed within the context of extended thermodynamics [44] and it is used for models of fast phase transformations [20].

Free Energy Density. Expanding the dependence of the free energy density on the concentration gradients and diffusion flux, one gets [45]

$$
\begin{gathered}
f(c, \nabla c, \mathbf{J})=f(c, 0,0)+\nabla c \cdot\left(\frac{\partial f}{\partial \nabla c}\right)_{\nabla c=0}+\frac{(\nabla c)^{2}}{2}\left(\frac{\partial^{2} f}{\partial(\nabla c)^{2}}\right)_{\nabla c=0}+\ldots \\
+\mathbf{J} \cdot\left(\frac{\partial f}{\partial \mathbf{J}}\right)_{\mathrm{J}=\mathbf{0}}+\frac{J^{2}}{2}\left(\frac{\partial^{2} f}{\partial J^{2}}\right)_{J=0}+\ldots
\end{gathered}
$$

The following points regarding Eq. (23) can be accepted. First, we define $f(c, 0,0)=f_{h}(c)$ as the free energy density of a homogeneous system with no gradients and fluxes. Second, the term $\nabla c \cdot \partial f / \partial(\nabla c)$ must be zero because the free energy of the system does not depend on the sign of the concentration gradient. Third, one can accept 
that the derivative of the free energy with respect to the diffusion flux is linear by the flux: $\partial f / \partial \mathbf{J}=\left(\alpha_{J} / 2\right) \mathbf{J}$ as approximation consistent with an extended thermodynamics [21]. And fourth, the next terms of expansion (23) can be omitted as a nonlinear terms in flux J. Therefore, Eq. (23) can be rewritten as

$$
f(c, \nabla c, \mathbf{J})=f_{h}(c)+\frac{r_{c}^{2}}{2}(\nabla c)^{2}+\frac{\alpha_{J}}{2} \mathbf{J} \cdot \mathbf{J},
$$

where $r_{c}^{2}=\left(\partial^{2} f / \partial(\nabla c)^{2}\right)_{\nabla c=0}$ and coefficient $\alpha_{J}$ is a characteristic of non-Fickian diffusion which assumed to be [21]

$$
\alpha=\frac{\tau_{D}}{T D}\left(\frac{\partial \mu}{\partial c}\right)_{T=c o n s t}
$$

with a difference $\mu$ of the chemical potentials for both chemical components. Within the limits of instant relaxation, i.e., $\tau_{D} \rightarrow 0$, the term with fluxes vanishes and Eq. (24) gives the free energy density $f(c, \nabla c)$ of the standard (Ginzburg-Landau or Cahn-Hilliard) form applicable for local equilibrium system.

Interpretation of Free Energy for Local Nonequilibrium States. Going beyond local equilibrium requires re-examination in depth such basic and conceptually relevant concepts as entropy, temperature, pressure or chemical potential under more general circumstances $[21,46]$. Therefore, free energy density (24) has to be interpreted in terms of a local thermodynamic potential [47, 48].

Equation (24) defines thermodynamic potential with both local equilibrium contribution $f_{h}(c)$ and purely local nonequilibrium contribution $(\alpha / 2) \mathbf{J} \cdot \mathbf{J}$ (under spatial inhomogeneity defined by the gradient term). Hence, for the local equilibrium part $f_{h}(c)$ a local ergodicity (i.e. the system needs to sample the phase space) is true. However, as soon as we postulated diffusion flux with a finite relaxation time, this means that the local nonequilibrium contribution $\alpha \mathbf{J} \cdot \mathbf{J}$ reflects the existence of a slow physical process, which is the jump of solute atoms [40]. Considering ergodicity of a phase space for nonequilibrium situation, one may well refer to statistical effects in fast spinodal decomposition due to existence of many particles (atoms and molecules) within local volumes. Since the liquid demixing proceeds very fast, the particles have no time enough to sample all the phase space. Thus, the number of microstates accessible to each of them will be lower than in equilibrium. This will imply an increasing in the free energy with respect to the local equilibrium contribution $f_{h}(c)$. This is one of the ways to interpret the nonequilibrium contribution $(\alpha / 2) \mathbf{J} \cdot \mathbf{J}$ to the free energy (24) that is the simplest conceivable way to express such increasing in the free energy. 
From the phenomenological pint of view, a purely non-equilibrium contribution $(\alpha / 2) \mathbf{J} \cdot \mathbf{J}$ to the free energy (or entropy) density for systems under spinodal decomposition controlled by atomic diffusion is explained as a kinetic energy. Thermodynamic interpretation has been recently made [49] for this contribution in the framework of multicomponent fluids and of dipolar systems having magnetic moments with non-vanishing inertia. A model, which takes this kinetic contribution, is called 'hyperbolic' model of spinodal decomposition, because it leads to the constitutive equation of hyperbolic type. In the limit of instantaneous relaxation, i.e. $\tau_{D} \rightarrow 0$, the term $\alpha \mathbf{J}$. J vanishes and Eq. (24) gives the free energy density $f_{h}(c, \nabla c)$ of Cahn-Hilliard's form $[12,13]$ applicable for local equilibrium system.

Free Energy Functional. Taking Eq. (24), the total Helmholtz free energy as a free energy functional is given by

$$
F(c, \nabla c, \mathbf{J})=\int_{v}\left[f_{h}(c)+\frac{r_{c}^{2}}{2}(\nabla c)^{2}+\frac{\alpha_{J}}{2} \mathbf{J} \cdot \mathbf{J}\right] d V,
$$

where $V$ is a sub-volume of the system. Evolution of $F(c, \nabla c, \mathbf{J})$ with time $t$ is described by

$$
\frac{d F}{d t}=\left(\frac{d F}{d t}\right)_{\mathrm{ex}}+\left(\frac{d F}{d t}\right)_{\mathrm{in}},
$$

where $(d F / d t)_{\text {ex }}$ is the external exchange of the free energy and $(d F / d t)_{\text {in }}$ is the internal change of the free energy inside the system. The latter is defined as a dissipative function. Using the procedure described in Refs [19, 20] and applied to Eq. (26), one can obtain

$$
\begin{gathered}
\left(\frac{d F}{d t}\right)_{\mathrm{ex}}=\int\left[r_{c}^{2}\left(\nabla_{n} c\right) \frac{\partial c}{\partial t}+\left(-f_{c^{\prime}}+r_{c}^{2} \nabla^{2} c\right) J_{n}\right] d \Omega, \\
\left(\frac{d F}{d t}\right)_{\mathrm{in}}=\int \mathbf{J} \cdot\left[\nabla\left(f_{c}^{\prime}-r_{c}^{2} \nabla_{n}^{2} c\right)+\alpha_{J} \frac{\partial \mathbf{J}}{\partial t}\right] d V,
\end{gathered}
$$

where $\Omega$ is the outer surface of sub-volume $V, J_{n}$ is the diffusion flux pointed by the normal vector $\mathbf{n}$, and $f_{c}^{\prime}=\partial f_{h} / \partial c$. As it follows from Eq. (29), the dissipative function includes the term $\alpha_{J} \partial \mathbf{J} / \partial t$, which has a clear physical meaning: far from equilibrium, the diffusion flux provides additional ordering that is leading to increasing of the dissipation.

Around a steady state, dissipative function (29) must decrease in time, so that the free energy of the entire system is decreasing. This condition implies a relation between fluxes and forces, which is, in the simplest case, assumed to be linear [21]. For Equation (29), it gives the following evolution equation for the diffusion flux 


$$
\mathbf{J}=-M \nabla\left(f_{c}^{\prime}-r_{c}^{2} \nabla^{2} c\right)-M \alpha_{J} \frac{\partial \mathbf{J}}{\partial t},
$$

where $M$ is the atomic mobility. Together with the atomic mass balance

$$
\frac{\partial c}{\partial t}=-\nabla \cdot \mathbf{J}
$$

Eq. (30) leads to the following governing equation

$$
\tau_{D} \frac{\partial^{2} c}{\partial t^{2}}+\frac{\partial c}{\partial t}=\nabla \cdot\left[M \nabla\left(f_{c}^{\prime}-r_{c}^{2} \nabla^{2} c\right)\right]
$$

which is the same that it has been previously derived from the entropy functional $[19,20]$. Equation (32) is a general partial differential equation of a hyperbolic type with the decomposition delay described by the term $\tau_{D} \partial^{2} c / \partial t^{2}$. It allows for describe both diffusion mechanism and wave propagation of chemical components.

A natural boundary condition, originating from external exchange of the free energy (28), is given by

$$
\varepsilon_{c}^{2} \frac{\partial c}{\partial t} \nabla_{n} c-\left(f_{c}^{\prime}-\varepsilon_{c}^{2} \nabla^{2} c\right) J_{n}=0
$$

where $J_{n}$ and $\nabla_{n} c$ are the projections of the diffusion flux and 'nabla'operator, respectively, on the normal vector to the boundary of the volume $v_{0}$. Equation (33) represents a dynamical boundary condition, which shows that the product $(\partial c / \partial t) \nabla_{n} c$ should be balanced with the product $\mu_{c} J_{n}$ on the boundary of the subvolume $V$. From this, in particular, it follows that if the concentration is fixed, $c=$ const, then the flux is absent, $J_{n}=0$, on the boundary $\Omega$. In the standard parabolic situation described by the Cahn-Hilliard equation $\left(\tau_{D} \rightarrow 0\right)$, one has proportionality between the flux and concentration gradient, $J_{n} \propto \nabla_{n} c$, and they both can be cancelled from Eq. (33). In this case, equation (33) transforms, with some scaling constant, into the known boundary condition analyzed by Miranville and Zelik (see Eqs (2) and (1.2) from Refs [50, 51], respectively). Hence, Eq. (32), endowed with a dynamic boundary condition (33), is a general partial differential equation of hyperbolic type with the decomposition delay described by the inertial term $\tau_{D} \partial^{2} c / \partial t^{2}$. Mathematically, the problem of CahnHilliard equation with the term $\tau_{D} \partial^{2} c / \partial t^{2}$, endowed with proper boundary conditions, has been studied in one-, two-, and threedimensions [23-25] to establish existence of the global and exponential attractors for different phase spaces.

Because we focus on the analysis of the initial stages of decomposition described by Eq. (32) (i.e., when the large concentration gradients exist and short periods of time are important) one may neglect all 
terms not linear in $c$. This one yields

$$
\tau_{D} \frac{\partial^{2} c}{\partial t^{2}}+\frac{\partial c}{\partial t}=M f_{c c}^{\prime \prime} \nabla^{2} c-M r_{c}^{2} \nabla^{4} c
$$

where $f_{c c}{ }^{\prime \prime}=\partial^{2} f_{h} / \partial c^{2}$. As $\tau_{D} \rightarrow 0$, Eq. (34) transfers into the classic Cahn-Hilliard equation [12, 13]. In the present form, Eq. (34) can be considered as a modified Cahn-Hilliard equation, which is a linearized partial differential equation of a hyperbolic type. This equation is true for spinodal decomposition with local nonequilibrium diffusion (diffusion with relaxation of the solute flux). Such type of decomposition is expected for short periods of time, large characteristic velocities of process, large concentration gradients, or under deep supercoolings.

\subsection{Dispersion Relation and Speeds for Atomic Diffusion}

Main characteristics of diffusion can be found from dispersion analysis of the linearized hyperbolic Cahn-Hilliard equation (34). These are the phase speed that characterizes propagation of a single (selected) harmonic, the group speed, which is characteristic of a wave packet, critical wavelength for decomposition, and critical time for instability, which both characterize developing coherent structure in decomposition [52].

We consider the elementary exponential solution of Eq. (34) in the following form

$$
c(z, t)-c_{0}=a_{k} \exp [i(k z-\omega(k) t)],
$$

where the dispersion relation $\omega(k)$ is given by

$$
\omega(k)=-\frac{i}{2 \tau_{D}} \pm\left(\frac{M k^{2}\left(f_{c c}^{\prime \prime}+r_{c}^{2} k^{2}\right)}{\tau_{D}}-\frac{1}{4 \tau_{D}^{2}}\right)^{1 / 2} .
$$

The upper and lower signs for $\omega(k)$ in Eq. (36) correspond to the branches, which are responsible for the wave propagation in the positive and negative $z$-directions, respectively. Qualitative behaviour for $\omega(k)$ is shown in Fig. 2.

It can be seen that the real part of $\omega$ begins to exist only from some critical value, $k=k_{0}$ (Fig. $2, a$ ). This value defines confluence of two branches for imaginary part of $\omega$ (Fig. $2, b$ ). In addition, one can define other two critical values for the wave-vector $k$. The critical value $k=k_{c}$ defines a point from which $\omega$ takes positive values of its imaginary part (Fig. $2, b$ ). For $k>k_{c}$, solution (35) exponentially grows in time and decomposition begins to proceed irreversibly. The critical value $k=k_{m}$ gives a maximal positive value for $\omega$ (Fig. 2, b). Fre- 


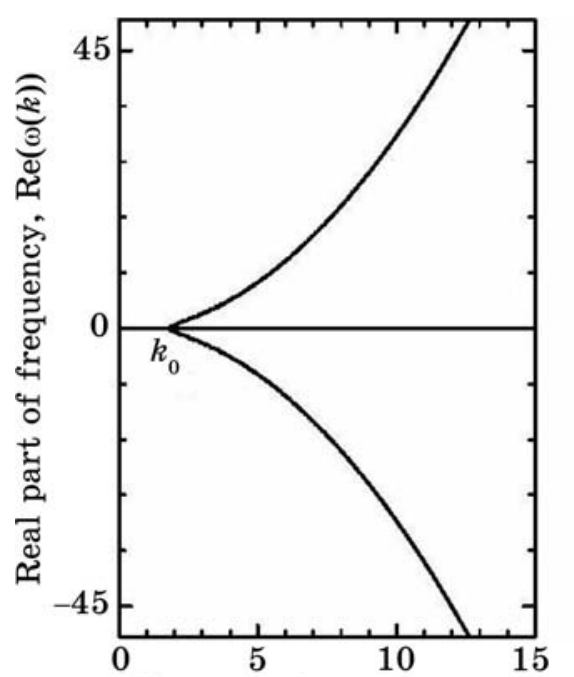

$k$, dimensionless wave vector

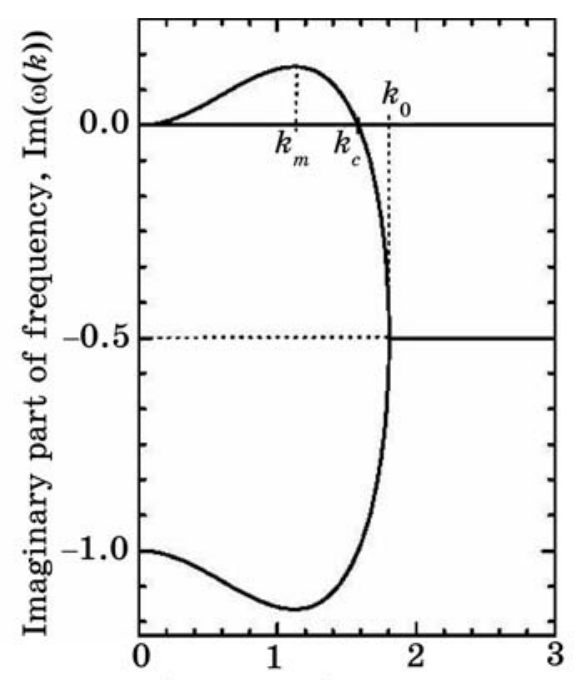

$k$, dimensionless wave vector

$b$

Fig. 2. Dispersion relations for hyperbolic Cahn-Hilliard equation; Eq. (36). (a) Real part of frequency, $\mathfrak{R}(\omega(k))$. (b) Imaginary part of frequency, $\mathfrak{I}(\omega(k))$.

quency $\omega\left(k_{m}\right)$ defines the mostly unstable mode with which pattern evolves during phase decomposition.

Within the local equilibrium limit $\tau_{D} \rightarrow 0$, Eq. (36) arrives to the following approximation

$$
\omega(k) \approx-\frac{i}{2 \tau_{D}}\left[1 \pm\left(1-2 \tau_{D} M k^{2}\left(f_{c c}^{\prime \prime}+r_{c}^{2} k^{2}\right)\right)\right] .
$$

Equation (37) shows that one of the roots is going to $-\infty$ along imaginary axis by the law $\omega(k) \propto i / \tau_{D}$. This leads to exponential decay of the solution (35). The second root of Eq. (37) is finite and it is equivalent to classic Cahn-Hilliard relation

$$
\omega(k) \approx-i M k^{2}\left(f_{c c}^{\prime \prime}+r_{c}^{2} k^{2}\right) .
$$

Thus, local equilibrium limit for dispersion relation (36) gives two different roots: the first one is diverges and the second one approaches dispersion relation (38) of Cahn and Hilliard.

Phase Speed. The values of the wave vector $k_{0}$ above which relation (36) has the real part, Fig. 2, $a$, is found from condition

$$
k_{0}^{2}=\frac{1}{2 r_{c}^{2}}\left(\sqrt{\left(f_{c c}{ }^{\prime \prime}\right)^{2}+\frac{r_{c}^{2}}{\tau_{D} M}}-f_{c c}^{\prime \prime}\right) .
$$


For $k^{2}>k_{0}^{2}$ and real values of $\omega(k)$, one can obtain from Eq. (36) the phase speed:

$$
v_{p}=\Re(\omega) / \Re(k)=\tau_{D}^{-1}\left(\tau_{D} M\left(f_{c c}^{\prime \prime}+r_{c}^{2} k^{2}\right)-(2 k)^{-2}\right)^{1 / 2},
$$

which may propagate in both positive and negative spatial directions. The speed $v_{p}$ incorporates a motion for one of separated single harmonics. It can be compared with the predictions of the partial differential equation of a hyperbolic type for solute diffusion without phase separation. Indeed, analysis of dispersion relation for mass transport equation $\tau_{D} \partial^{2} c / \partial t^{2}+\partial c / \partial t=D \nabla^{2} c$ of a hyperbolic type leads to the following expression [53]:

$$
v_{p}=\left(\frac{2 D}{\tau_{D}+\left(\tau_{D}^{2}+\omega_{c}^{-2}\right)^{1 / 2}}\right)^{1 / 2} .
$$

Taking into account that $r_{c}=0$, for the zero spatial atomic correlation, $M f_{c c}{ }^{\prime \prime}=D$ is the diffusion coefficient in Eq. (40), we use the relation $k \propto \omega$ for high frequency of disturbances' propagation. Then, both expressions (40) and (41) lead to the same result

$$
v_{p}=\left(D / \tau_{D}\right)^{1 / 2}=V_{D} \quad \text { with } \quad \omega \rightarrow \infty .
$$

In Equation (42), the phase speed $v_{p}$ is equal to solute diffusion speed $V_{D}$, which is a maximal speed for propagation of the solute diffusion disturbance (profile).

Imaginary part of the phase speed, $\mathfrak{I}\left(v_{p}\right)=-i /\left(2 \tau_{D} k\right)$, specifies the amplification rate for a given harmonic. With $k<k_{0}$, harmonics do not move with possible changing of their own amplitudes. For both real and imaginary parts of $v_{p}$ (with $k>k_{0}$ ), the harmonics move and change their own amplitudes. The behaviour is shown in Fig. 3 for $v_{p}(k)$.

Group Speed. Concentration disturbances propagating by diffusion can be considered as an undistorted wave packet moving with the group speed given by

$$
\frac{\partial \omega(k)}{\partial k}= \pm W(k) .
$$

Using Eq. (36), calculation of the group speed $W$ gives

$$
W(k)=\frac{2 k M\left(f_{c c}^{\prime \prime}+2 r_{c}^{2} k^{2}\right)}{\left(4 \tau_{D} k^{2} M\left(f_{c c}^{\prime \prime}+r_{c}^{2} k^{2}\right)-1\right)^{1 / 2}} .
$$

Dependence $W(k)$ is shown in Fig. 3. It specifies a speed for concentration profiles envelope. One may see, as for the phase speed $v_{p}$, the real values for $W$ given by Eq. (44) exist only at $k>k_{0}$. In contrast with the 


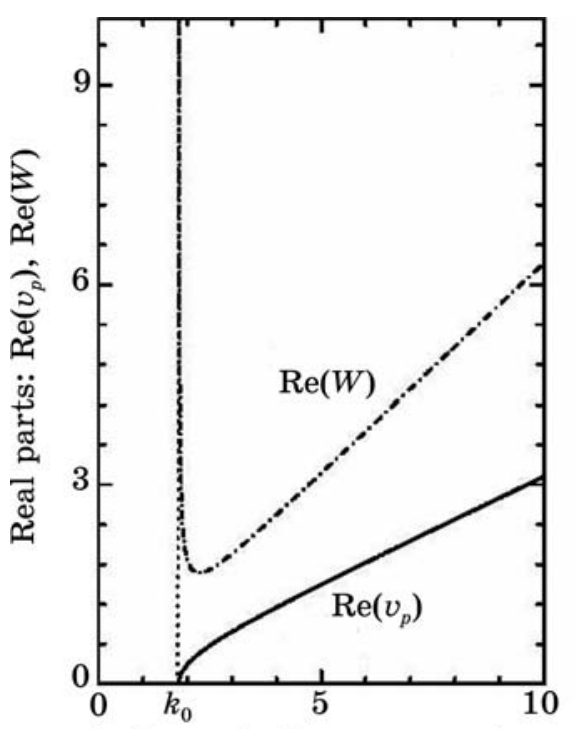

$k$, dimensionless wave vector $a$

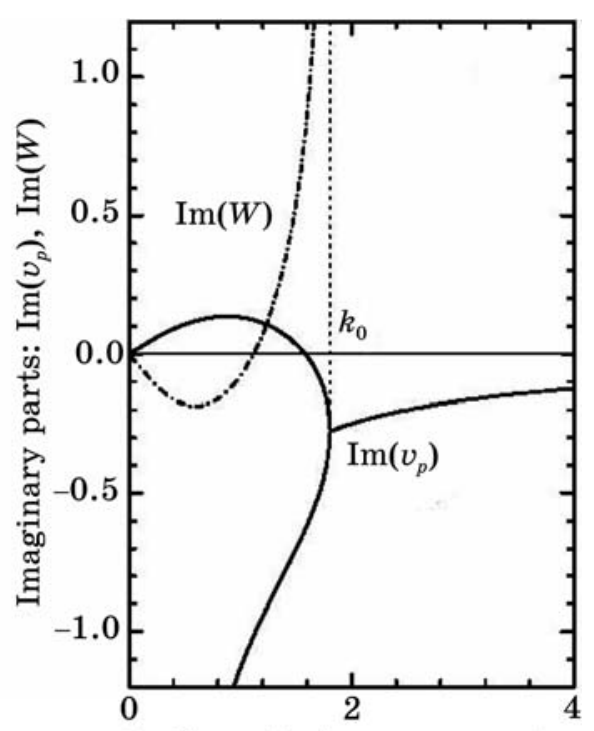

$k$, dimensionless wave vector $b$

Fig. 3. Phase and group speeds for hyperbolic Cahn-Hilliard equation. (a) Real part $\mathfrak{R}\left(v_{n}\right)$ of phase speed (solid line) and real part $\mathfrak{R}(W)$ of group speed (dashed-dotted line). (b) Imaginary part $\mathfrak{I}\left(v_{n}\right)$ of phase speed (solid line), and imaginary part $\mathfrak{I}(W)$ of group speed (dashed-dotted line).

behaviour of $v_{p}$, the imaginary part of $W(k)$ may exist only at $k<k_{0}$.

TABLE 1. Predictions for characteristic speeds of diffusion.

\begin{tabular}{|c|c|c|}
\hline Equation & Phase speed $v_{p}$ & Group speed $W$ \\
\hline $\begin{array}{l}\text { Parabolic } \\
\text { diffusion } \\
\text { equation }\end{array}$ & $-i k D$ with $\omega=-i k^{2} D$ & $-2 i k D$ \\
\hline $\begin{array}{l}\text { Hyperbolic } \\
\text { diffusion } \\
\text { equation }\end{array}$ & $\frac{-i \pm \sqrt{4 \tau_{D} D k^{2}-1}}{2 \tau_{D} k}$ & $\frac{2 k D}{\sqrt{4 \tau_{D} D k^{2}-1}}$ \\
\hline $\begin{array}{l}\text { Parabolic } \\
\text { Cahn- } \\
\text { Hilliard } \\
\text { equation } \\
\end{array}$ & $-i M k\left(f_{h}^{\prime \prime}+r_{c}^{2} k^{2}\right)$ & $-2 i M k\left(f_{h}^{\prime \prime}+2 r_{c}^{2} k^{2}\right)$ \\
\hline $\begin{array}{l}\text { Hyperbolic } \\
\text { Cahn- } \\
\text { Hilliard } \\
\text { equation } \\
\end{array}$ & $-\frac{i}{2 k \tau_{D}}\left(1 \pm \sqrt{1-4 \tau_{D} M k^{2}\left(f_{h}^{\prime \prime}+r_{c}^{2} k^{2}\right)}\right)$ & $\frac{2 k M\left(f_{h}^{\prime \prime}+2 r_{c}^{2} k^{2}\right)}{\sqrt{4 \tau_{D} k^{2} M\left(f_{h}^{\prime \prime}+r_{c}^{2} k^{2}\right)-1}}$ \\
\hline
\end{tabular}


Analysis of standard parabolic and hyperbolic diffusion equations [53] as well as parabolic and hyperbolic equations for spinodal decomposition [52], presented in this Subsection for diffusion speeds, leads to comparison of both approximations summarized in Table 1.

\subsection{Critical Parameters for Hyperbolic Decomposition}

\subsubsection{Critical Wavelength for Decomposition}

Cahn [13] has found a critical wavelength $\lambda_{c}$, above which infinitesimal sinusoidal fluctuation of concentration is irreversibly grown. Particularly, he confirmed the concept of Hillert [54] that $\lambda_{c} \rightarrow \infty$ with approaching the spinodal, at which one has $\partial^{2} f / \partial c^{2}=0$.

To find the critical wavelength for decomposition under local nonequilibrium diffusion, we expand $f_{h}(c)$ in Eq. (26) about some concentration $c_{0}$ that is

$$
f_{h}(c)=f\left(c_{0}\right)+\left(c-c_{0}\right)\left(\frac{d f_{h}}{d c}\right)_{c=c_{0}}+\frac{\left(c-c_{0}\right)^{2}}{2}\left(\frac{d^{2} f_{h}}{d c^{2}}\right)_{c=c_{0}}+\ldots
$$

The composition is represented along the $z$-axis by a series of sinusoidal waves with components of the following form

$$
c-c_{0}=a_{c} \cos \left(k_{z} z\right),
$$

where $a_{c}$ is the amplitude and $k_{z}$-the frequency of concentration wave.

Substituting Eq. (46) into Eq. (45), we perform integration of the functional (26) over the volume $v$. Then, for the difference of the Helmholtz free energy, $\Delta F=F(c, \nabla c, \mathbf{J})-\int f_{h}\left(c_{0}\right) d V$, between a system with concentration (46) and a homogeneous system, respectively, one gets:

$$
\frac{\Delta F}{V}=\frac{a_{c}^{2}}{4}\left[f_{c c}^{\prime \prime}+r_{c}^{2} k_{z}^{2}\right] .
$$

For the reasonable cases of the positive surface tension, $r_{c}^{2}>0$, one can consider two important points.

First, with $f_{c c}^{\prime \prime}>0$ the solution is stable against fluctuation of concentration of any wavelength: the free energy only increases in this case, $\Delta F>0$.

Second, with $f_{c c}{ }^{\prime \prime}<0$ the solution is unstable with respect to the critical wavelength for decomposition, which can merely be found by taking the zero value for the square bracket in Eq. (47): 


$$
\lambda_{c}=\frac{2 \pi}{k_{c}}=2 \pi\left(\frac{r_{c}^{2}}{-f_{c c}^{\prime \prime}}\right)^{1 / 2} .
$$

with the critical value for wave vector given by

$$
k_{c}=\left(-f_{c c}^{\prime \prime} / r_{c}^{2}\right)^{1 / 2}
$$

Therefore, with $d^{2} f_{h} / d c^{2}<0$ and for $\lambda>\lambda_{c}$, the free energy decreases, $\Delta F<0$, and decomposition starts to proceed. Equation (49) clearly shows that as the composition tends to the values lying in the spinodal, $\partial^{2} f / \partial c^{2}=0$, the critical wavelength approaches to infinity, $\lambda_{c} \rightarrow \infty[13,54]$.

\subsubsection{Amplification Rate of Decomposition}

Consider a real part of the solution (35) in the following form:

$$
c-c_{0}=a_{+} \cos (k z) \exp \left(\omega_{+} t\right)+a_{-} \cos (k z) \exp \left(\omega_{-} t\right) .
$$

In this solution, signs 'plus' and 'minus' correspond to growing or decaying solutions, respectively, in time. Substitution of Eq. (35) into Eq. (34) defines a real part of the frequency as follows

$$
\omega_{ \pm}=\left(2 \tau_{D}\right)^{-1}\left[-1 \pm\left(1-4 k^{2} \tau_{D} M\left(f_{c c}^{\prime \prime}+r_{c}^{2} k^{2}\right)\right)^{1 / 2}\right] \text {. }
$$

After expanding, the square root in Eq. (51) for $4 k^{2} \tau_{D} M\left[f_{c c}^{\prime \prime}+\right.$ $\left.+r_{c}^{2} k^{2}\right] \leq 1$ one gets in the local equilibrium limit the expression:

$$
\lim _{\tau_{D} \rightarrow 0} \omega_{+}=k^{2} M\left(f_{c c}^{\prime \prime}+r_{c}^{2} k^{2}\right),
$$

which is the kinetic amplification rate obtained by Cahn [13] for purely diffusion regime. Therefore, Eq. (51) can be interpreted as the kinetic amplification rate for both dissipative and propagative regimes of atomic transport described by Eq. (34).

From the amplification rate $\omega_{+}$of decomposition, the maximum can be obtained by differentiation of Eq. (51) with respect to $k_{z}$. The extremum condition, $\partial \omega_{+} / \partial k_{z}=0$, gives maximum frequency

at

$$
\omega_{m}\left(k_{m}\right)=\left(2 \tau_{D}\right)^{-1}\left[-1+\left(1+\tau_{D} M f_{c c}^{\prime \prime} / r_{c}^{2}\right)^{1 / 2}\right]
$$

$$
k_{m}=\left(-f_{c c}^{\prime \prime} /\left(2 r_{c}^{2}\right)\right)^{1 / 2} \quad \text { with } \quad f_{c c}^{\prime \prime}<0
$$


From Eq. (54), there follows that maximum wavelength, $\lambda_{m}=2 \pi / k_{m}$, is equal to

$$
\lambda_{m}=2 \pi\left(\frac{2 r_{c}^{2}}{-f_{c c}^{\prime \prime}}\right)^{1 / 2} .
$$

Consequently, maximum amplification rate allows for the wavelength (55) greater in exactly $\sqrt{2}$ times the critical wavelength (49) of instability against fluctuations of concentration. This result coincides with Cahn and Hilliard's results for purely diffusion regime.

\subsubsection{Critical Time for Instability}

Let us evaluate the time of transitive period from the beginning of instability (with the beginning of growth of infinitesimal perturbation) up to the arriving into the new metastable state. For the fastest growth of infinitesimal perturbation, the maximal frequency $\omega\left(k_{m}\right)$ is responsible. Therefore, substitution of Eq. (48) into dispersion relation (36) leads to [52]

$$
\omega_{m}\left(k_{m}\right)=-\frac{i}{2 \tau_{D}}\left[1 \pm\left(1+M \tau_{D}\left(f_{c c}^{\prime \prime}\right)^{2} / r_{c}^{2}\right)^{1 / 2}\right] .
$$

Equation (56) adopts both real and imaginary parts for $\omega$. Using maximal frequency (56), solution (35) can be rewritten as

where

$$
c(z, t)-c_{0}=a_{k} \exp (i k z) \exp \left(t / t_{c}\right),
$$

$$
t_{c}=\frac{2 \tau_{D}}{\left(1+M \tau_{D}\left(f_{c c}^{\prime \prime}\right)^{2} / r_{c}^{2}\right)^{1 / 2}-1}
$$

is the time for developing coherent structure.

Within the local equilibrium limit, $\tau_{D} \rightarrow 0$, we expand square root in Eq. (58) for $M \tau_{D}\left(f_{c c}^{\prime \prime}\right)^{2} / r_{c}^{2}=1$. One gets the following approximation

$$
t_{c} \approx \frac{4 r_{c}^{2}}{M\left(f_{c c}^{\prime \prime}\right)^{2}},
$$

which can be found from the predictions of pure diffusion theory (parabolic transport equation) of Cahn and Hilliard. As a result, comparative analysis for parabolic and hyperbolic equations in spinodal decomposition is given in Table 2 for dispersion relations, critical wavelengths and times for instability. 
TABLE 2. Predictions of parabolic and hyperbolic models.

\begin{tabular}{|c|c|c|}
\hline Expression for & $\begin{array}{c}\text { Parabolic Cahn-Hilliard } \\
\text { equation }\left(\tau_{D} \rightarrow 0\right)\end{array}$ & Hyperbolic Cahn-Hilliard equation \\
\hline \multirow{2}{*}{$\begin{array}{l}\text { Dispersion } \\
\text { relation, } \omega(k)\end{array}$} & \multirow{2}{*}{$-i M k^{2}\left(f_{c c}^{\prime \prime}+r_{c}^{2} k^{2}\right)$} & \multirow{2}{*}{$-\frac{i}{2 \tau_{D}} \pm\left(\frac{M k^{2}\left(f_{c c}^{\prime \prime}+r_{c}^{2} k^{2}\right)}{\tau_{D}}-\frac{1}{4 \tau_{D}^{2}}\right)^{1 / 2}$} \\
\hline & & \\
\hline $\begin{array}{l}\text { Critical wave- } \\
\text { length, } \\
\lambda_{c}=2 \pi / k_{c}\end{array}$ & $2 \pi\left(r_{c}^{2} /\left(-f_{c c}^{\prime \prime}\right)\right)^{1 / 2}[13]$ & $2 \pi\left(r_{c}^{2} /\left(-f_{c c}^{\prime \prime}\right)\right)^{1 / 2}$ \\
\hline \multirow{2}{*}{$\begin{array}{l}\text { Amplification } \\
\text { rate, } \omega_{+}\end{array}$} & \multirow{2}{*}{$k^{2} M\left(f_{c c}^{\prime \prime}+r_{c}^{2} k^{2}\right)[13]$} & $2 \tau_{D}$ \\
\hline & & $\left(1-4 k^{2} \tau_{D} M\left(f_{c c}^{\prime \prime}+r_{c}^{2} k^{2}\right)\right)^{1 / 2}-1$ \\
\hline $\begin{array}{l}\text { Maximal wave- } \\
\text { length, } \\
\lambda_{m}=2 \pi / k_{m}\end{array}$ & $2 \pi\left(2 r_{c}^{2} /\left(-f_{c c}^{\prime \prime}\right)\right)^{1 / 2}[13]$ & $2 \pi\left(2 r_{c}^{2} /\left(-f_{c c}^{\prime \prime}\right)\right)^{1 / 2}$ \\
\hline Critical time for & & $2 \tau_{D}$ \\
\hline instability, $t_{c}$ & $4 r_{c}^{2} /\left(M\left(f_{c c}\right)^{2}\right)[52]$ & $\left(1+M \tau_{D}\left(f_{c c}^{\prime \prime}\right)^{2} / r_{c}^{2}\right)^{1 / 2}-1$ \\
\hline
\end{tabular}

\subsubsection{Analysis of a Structure Function}

Different evolution of spinodally-decomposed systems exhibits different structures (see Fig. 10). Experimentally, a typical structure after spinodal decomposition is observed as random, interconnected patterns with a characteristic length-scale related to maximal amplification rate of decomposition [4]. Experimental observations using scattering show a broad Bragg-like peaks, from which information about quenched structure during spinodal decomposition and decomposition rate can be read off. For such measurements, a main characteristic for the intensity of scattering is a structure factor. Therefore, the structure factor can be taken as a parameter for characterization of analyzed evolutions and for verification of the model predictions with experimental data.

Consider the structure factor $S(\mathbf{k}, t)$, which describes the intensity of quasi-elastic scattering observed at time $t$ after the quenching from the initial temperature $T_{i}$ up to the final temperature $T_{f}$. The function $S(\mathbf{k}, t)$ can be interpreted as the respective correlation function of the concentration fluctuations, and it is defined as

$$
S(\mathbf{k}, t)=\langle\delta c(-\mathbf{k}, t) \delta c(\mathbf{k}, t)\rangle_{T_{f}} .
$$

To obtain expression for the time dependent structure factor (60), 
we linearize Eq. (34) in terms of concentration inhomogeneity $\delta c(\mathbf{r}, t)=c(\mathbf{r}, t)-c_{0}$. This one yields

$$
\tau_{D} \frac{\partial^{2}(\delta c)}{\partial t^{2}}+\frac{\partial(\delta c)}{\partial t}=M f_{c c}^{\prime \prime} \nabla^{2}(\delta c)-M r_{c}^{2} \nabla^{4}(\delta c) .
$$

Following the approach to the concentration fluctuations for the hyperbolic transport [40], Fourier transforms of $\delta c(-\mathbf{k}, t)$ and $\delta c(\mathbf{k}, t)$ is expressed as

$$
\begin{aligned}
& \delta c(-\mathbf{k}, t)=\int d \mathbf{r} \exp (i \mathbf{k} \cdot \mathbf{r}) \delta c(-\mathbf{r}, t), \\
& \delta c(\mathbf{k}, t)=\int d \mathbf{r} \exp (i \mathbf{k} \cdot \mathbf{r}) \delta c(\mathbf{r}, t) .
\end{aligned}
$$

Then, Eq. (61) for the structure factor (60) is given by

$$
\tau_{D} \frac{d^{2} S(\mathbf{k}, t)}{d t^{2}}+\frac{d S(\mathbf{k}, t)}{d t}=-M k^{2}\left(f_{c c}^{\prime \prime}+r_{c}^{2} k^{2}\right) S(\mathbf{k}, t) .
$$

To solve this equation, we multiply LHS and RHS by $\exp (i \omega t)$, where $\omega$ is a frequency of the concentration inhomogeneity. After some algebra, solution of Eq. (63) is given by

$$
\int_{0}^{\infty} \exp (-i \omega t) S(\mathbf{k}, t) d t=\frac{\tau_{D} d S(\mathbf{k}, 0) / d t+\left(1+\tau_{D} i \omega\right) S(\mathbf{k}, 0)}{i \omega-\tau_{D} \omega^{2}+M k^{2}\left(f_{c c}^{\prime \prime}+r_{c}^{2} k^{2}\right)} .
$$

Defining the spectral distribution of fluctuations as

$$
S(\mathbf{k}, \omega)=2 \Re \int_{0}^{\infty} \exp (-i \omega t) S(\mathbf{k}, t) d t
$$

one can find from Eq. (64) the spectral distribution for concentration fluctuations.

Equations (64) and (65) might describe modelled structure for hyperbolic scenario (with finite $\tau_{D}$ ) and parabolic scenario (for instant relaxation with $\tau_{D} \rightarrow 0$ ) and experimentally observed structure after quenching in spinodal decomposition. Such a description can give information about length scale of concentration fluctuations and, as a consequence, about maximal amplification rate of decomposition for given scenario.

\subsection{Comparison with Experimental Data}

The function of the amplification rate predicted by the hyperbolic model has been compared in Refs $[48,55]$ with experimental data of Andreev et al. on phase-separated glasses [56, 57]. The amplification rate (51) can be rewritten in the following form 


$$
\omega_{+}=\frac{\left[1+4 D \tau_{D} k^{2}\left(1-r_{c}^{2} k^{2} / f_{c c}^{\prime \prime}\right)\right]^{1 / 2}-1}{2 \tau_{D}}=\frac{\left[1+4 l_{D}^{2} k^{2}\left(1-l_{C}^{2} k^{2}\right)\right]^{1 / 2}-1}{2 \tau_{D}},
$$

where $l_{C}=r_{c} / \sqrt{-f_{c c}^{\prime \prime}}$ is the correlation length, $l_{D}=\left(D \tau_{D}\right)^{1 / 2}$ is the diffusion length, and $D=-M f_{c c}^{\prime \prime}$ is the diffusion constant.

In addition, one can assume that the free energy of a binary system can be replaced by

$$
f_{h}(T, c)=f_{0}\left[\left(T / T_{c}-1\right)\left(c-c_{c}\right)^{2}+B_{0}\left(c-c_{c}\right)^{4}\right],
$$

where $T_{c}$ and $c_{c}$ are the critical temperature and concentration, respectively, $T<T_{c}$, and $B_{0}>0$. Equation (67) is often used in analysis of kinetics of spinodal decomposition in glasses [58], and the parameters $f_{0}$ and $B_{0}$ are treated as phenomenological input parameters of the theory, which are fitted to experiment [10].

Figure 4 shows data for the relationship ' $\omega_{+} / k^{2}$ versus $k^{2}$, extracted from experiments on a binary phase-separated glass [56, 57]. They exhibit non-linear behaviour as predicted by Eqs (66) and (67) for the following material parameters: $D=2.3 \cdot 10^{-14} \mathrm{~cm}^{2} / \mathrm{s}$, $\tau_{D}=7.2 \cdot 10^{-11} \mathrm{~s}, \quad r_{c}=6.2 \cdot 10^{-8} \mathrm{~cm} \cdot \sqrt{J /\left(\mathrm{mole} \cdot \mathrm{cm}^{3}\right)}, \quad T / T_{c}=0.85$, $B_{0}=0.15, f_{0}=1.88 \cdot 10^{4} \mathrm{~J} /\left(\mathrm{mole} \cdot \mathrm{cm}^{3}\right)$, and $c-c_{c}=0.8$ mole frac-

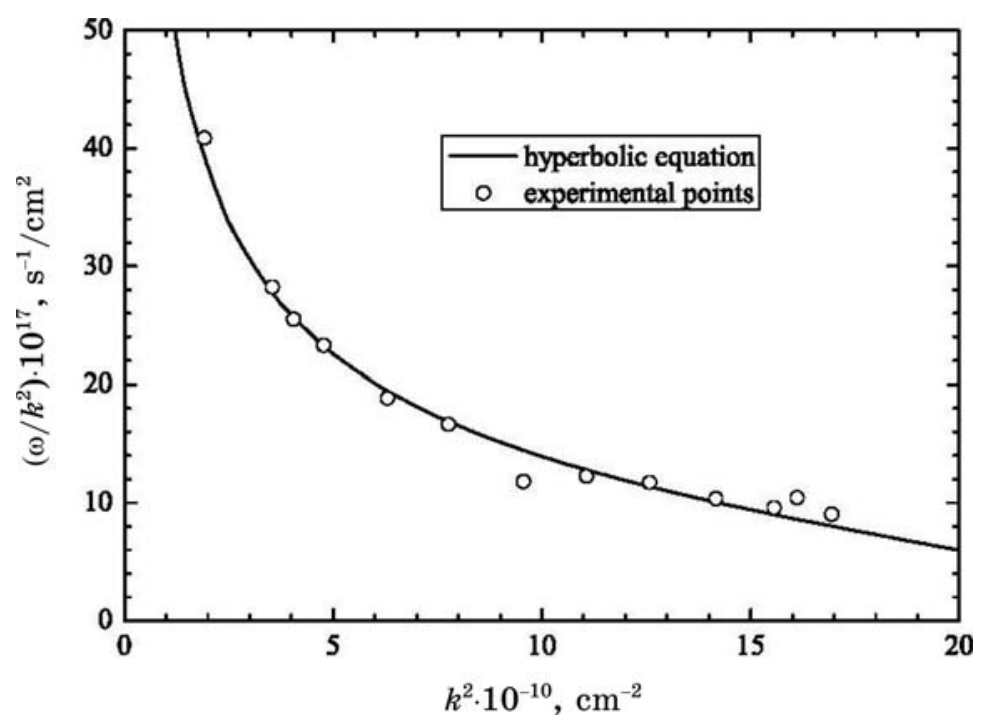

Fig. 4. Dependence $\omega_{+} / k^{2}$ upon $k^{2}$ given by the hyperbolic model (solid line; Eqs (66) and (67)) and scattering data of visible light (points, Refs [56, 57]). Experimental points were obtained on phase-separated $\mathrm{SiO}_{2}-12$ wt. $\% \mathrm{Na}_{2} \mathrm{O}$ glass at $T=803 \mathrm{~K}$. 
tion.

In Figure 4, it is shown that good agreement is achieved between theory and experiment. This result is due to the fact that both lengths, correlation length $l_{C}$ and diffusion length $l_{D}$, appear in the theory $[48,55]$ that is also shown by Eq. (66). The interplay between these two lengths, i.e. the ratio $l_{D} / l_{C}$, governs the transition as follows (in contrast with the linear Cahn-Hilliard-Cook model $[12,59]$ in which only correlation length $l_{C}$ is important in spinodal decomposition). With the increase of the correlation length $l_{C}$ in comparison with the diffusion length $l_{C}$, spinodal decomposition has the Cahn-Hilliard's scenario (described by linear or non-linear parabolic diffusion equation). With $l_{D} \approx l_{C}$, one can accept long-range interaction within the system and the Cahn-Hilliard's scenario takes effect. With $l_{D} \gg l_{C}$ (namely, with $l_{D} \geq 2 \sqrt{2} l_{C}$ [52]), shortrange interaction has effect and local nonequilibrium effect (such as relaxation of the diffusion flux to its steady state) plays dominant role in selection of the mode for decomposition. Thus, existence of these two length, existence of these two length, $l_{D}$ and $l_{C}$, makes the theory flexible enough to predict non-linear behaviour for amplification rate typically observed in experiments and to quantitatively describe experiments (Fig. 4).

\section{MODELLING OF SPINODAL DECOMPOSITION}

In this Section, we present main numeric procedures to be used in simulations of the spinodal decomposition in deterministic models of the hyperbolic type. Numerical approaches related for $1 D$ and $3 D$ simulations are presented in Subsections 3.1 and 3.2, respectively.

Features of hyperbolic spinodal decomposition can be observed in computational dynamics.

To model decomposition, Eqs (26), (30), and (31) are taken. From this system, the following dimensionless form of equations is as follows

$$
\begin{gathered}
\frac{\delta F}{\delta c}=-f_{c^{\prime}}+\left(\frac{r_{c}}{l_{D}}\right)^{2} \nabla^{2} c, \\
\frac{\partial \mathbf{J}}{\partial t}+\mathbf{J}=\nabla\left(\frac{\delta F}{\delta c}\right), \\
\frac{\partial c}{\partial t}=-\nabla \cdot \mathbf{J} .
\end{gathered}
$$

In these equations, the following scales are introduced: $M=D / f_{c c}{ }^{\prime \prime}$ is the mobility, $l_{D}=D / V_{D}$ the characteristic spatial length, $\tau_{D}$ the 
time scale as the diffusion relaxation time, and $V_{D}$ the solute diffusion speed as the scale for diffusion flux. To complete this system, the free energy density is chosen as a double-well potential

$$
f_{h}(c)=f_{0}(1-c)^{2} c^{2} / 4,
$$

which has minima at $c=0$ and $c=1$, and it has a maximum at $c=0.5$.

Boundary conditions are established from the previously obtained expression for the external exchange $(d F / d t)_{\mathrm{ex}}$ of the free energy: expression in square brackets of Eq. (28) must be zero. This condition implies the following equalities $\nabla_{n} c=0$ and $J_{n}=0$ on the boundary of the calculated domain. As a result, we arrive to a set of hyperbolic equations (68)-(71), which describes evolution by the hyperbolic model described in previous Section (in comparison with the parabolic model of Cahn and Hilliard using diffusion equation of a parabolic type).

\subsection{D Modelling}

For integration of equations (68)-(71), we use implicit Euler method of second order $O\left(\tau^{2}\right)$. Let us introduce the following notations

$$
p=\left.\frac{\partial c}{\partial t}\right|_{t=t^{n}+\frac{1}{2} \tau}, \quad s=\left.\frac{\partial J}{\partial t}\right|_{t=t^{n}+\frac{1}{2} \tau},
$$

where $\tau$ is the time step. Then one can define the system of equations for the time iterations in the following form

$$
c^{(n+1)}=c^{(n)}+p \tau, J^{(n+1 / 2)}=J^{(n)}+\frac{1}{2} s \tau, \tilde{c} \equiv c^{(n+1 / 2)}=c^{(n)}+\frac{1}{2} p \tau .
$$

Now, Eqs (68)-(71) can be rewritten as

$$
\left\{\begin{array}{l}
p+\frac{\partial}{\partial x}\left(J^{(n)}+\frac{1}{2} \tau s\right)=0, \\
s\left(1+\frac{1}{2} \tau\right)+J^{(n)}=-M(\tilde{c}) \frac{\partial}{\partial x} W, \\
W=f_{\tilde{c}}^{\prime}-\left(\frac{r_{c}}{l_{D}}\right)^{2} \frac{\partial^{2} c^{(n)}}{\partial x^{2}}-\frac{\tau}{2}\left(\frac{r_{c}}{l_{D}}\right)^{2} \frac{\partial^{2} p}{\partial x^{2}},
\end{array}\right.
$$

where $r_{c} / l_{D}$ is the ratio of correlation parameter $r_{c}$ and diffusion length $l_{D}=D / V_{D}$. Excluding $s$ from (74), one can obtain the final system of equations. This one yields 


$$
\left\{\begin{array}{l}
\frac{\tau}{2}\left(\frac{r_{c}}{l_{D}}\right)^{2} \frac{\partial^{2} p}{\partial x^{2}}+W=f_{\tilde{c}}^{\prime}-\left(\frac{r_{c}}{l_{D}}\right)^{2} \frac{\partial^{2} c^{(n)}}{\partial x^{2}} \\
\frac{\partial}{\partial x}\left(M(\tilde{c}) \frac{\partial}{\partial x} W\right)-\left(1+\frac{2}{\tau}\right) p=\frac{2}{\tau} \frac{\partial J^{(n)}}{\partial x} .
\end{array}\right.
$$

Equations (75) represent an elliptic set of equations. This system allows us to numerically solve Eqs (68)-(71) relatively the functions $p$ and $W$ using the following algorithm. Taking initial data for concentration $c(x, 0)=c_{0}\left(x_{k}\right)=c_{k}^{0}$ and diffusion flux $J(x, 0)=0=J_{k}^{0}$, variables $p$ and $W$ are obtained from the system (75). Then, new data for the concentration and the flux are found from the system (73) for the new time level. These are used for obtaining $p$ and $W$ from Eqs (75). This procedure is iterated in time to compute concentrations and diffusion fluxes in spinodal decomposition.

Note that the system (75) can be also used for solution of the Cahn and Hilliard's parabolic equation for spinodal decomposition. This procedure has to assume the steady-state diffusion flux, i.e. the flux needs infinite time for its time changing. Therefore, let $\tau \rightarrow \infty$ in the second equation of Eqs (75). This excludes from the numerical procedure the time dependence of the flux and the second order derivative of concentration with respect to time. In addition, the second equation in Eq. (73) has to be divided on $\tau$ with the further taking the same limit $\tau \rightarrow \infty$. This leads to equality $s=\partial J / \partial t=0$ that allows us to exclude the time dependence of flux from the suggested numeric algorithm.

Choosing a finite difference method and using approximation of the second order for coordinate $x$, elliptic system (75) can be tested against its computational stability. A linearized transfer-matrix $T(k, n)$ is obtained for deviations $\delta c$ and $\delta J$ from exact solutions of Eqs (75). This one yields

$$
\left(\begin{array}{c}
\delta c^{(n+1)} \\
\delta J^{(n+1)}
\end{array}\right)=T(k, n)\left(\begin{array}{c}
\delta c^{(n)} \\
\delta J^{(n)}
\end{array}\right),
$$

where

$$
T(k, n)=N_{0}\left(\begin{array}{cr}
-\frac{1}{2} M L \tau^{2} k^{2}\left(1+\frac{1}{2} \tau\right) & -i \tau k\left(1+\frac{1}{2} \tau\right) \\
-i M L k \tau\left(1+\frac{1}{2} \tau\right)^{2} & 1-\frac{1}{4} \tau^{2}\left(1+M L k^{2}(1+3 \tau)\right)
\end{array}\right),
$$

where $k$ is the wave vector, and factors $N_{0}$ and $L$ are obtained as

$$
N_{0}=\left(1+\tau / 2+\tau^{2} M L k^{2} / 4\right)^{-1}(1+\tau / 2)^{-1},
$$




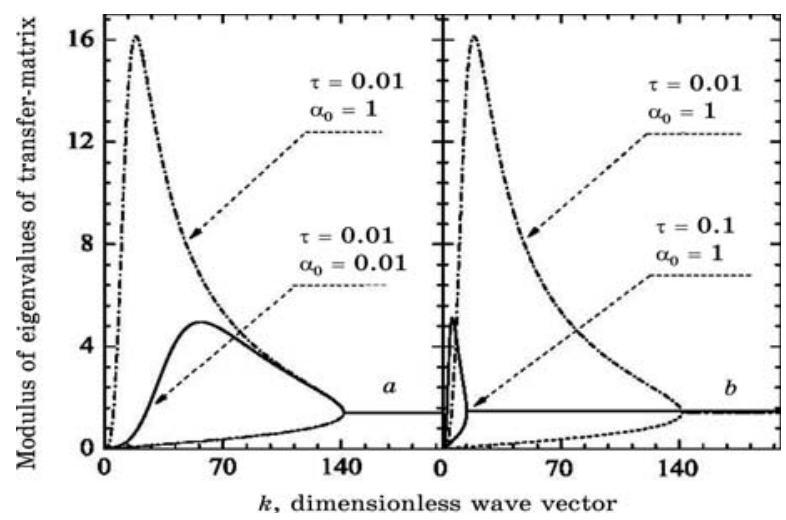

Fig. 5. Typical dependence of modulus of eigenvalues for transfer-matrix $\widehat{T}(k, n)$ on dimensionless wave vector $k$ with the scale of $2 \pi l_{D}$. Region of computational stability lies below unity for the eigenvalues of transfer matrix. Dependence is shown at: $(a)$ different values $\alpha_{0}=r_{c} / l_{D}$ that is the ratio of correlation parameter $r_{c}$ and diffusion length $l_{D}=D / V_{D}$, and $(b)$ different $\tau$.

and $L(n, k)=f_{\tilde{c} \tilde{c}}^{\prime \prime}+\left(r_{c} / l_{D}\right) k^{2}$. The stability condition requires that, for given wave vector $k$, the eigenvalues for transport matrix (77) have to be not greater than unity. Qualitative dependence of modulus of eigenvalues for transfer-matrix $\widehat{T}(k, n)$ from the wave vector $k$ is present in Fig. 5. This dependence is shown at different values of parameters of the time step $\tau$ and relation $\alpha_{0}=r_{c} / l_{D}$ between the correlation parameter $r_{c}$ and diffusion length $l_{D}=D / V_{D}$. One can see that numerical scheme is conditionally stable. The condition of stability is formed mainly by a largest possible value of wave vector and weakly depends on other parameters, particularly, from the time step $\tau$.

Dynamics of spinodal decomposition is presented in Figs. 6 and 7 for the material and computational parameters summarized in Table 3. The dynamics is shown in spatial changing of concentration profiles for a given time step (Figs. 6, 7, $a-d$ ). After formation of quasisinusoidal profile from an initially random distribution, this distribution becomes unstable in further separation due to up-hill diffusion between decomposing phases (Fig. 6, $a-b$ ). This unstable situation evolves much more faster for the system described by Cahn-Hilliard equation than for the local nonequilibrium system described by hyperbolic equation (Fig. 6, $b-c$ ). It occurs, generally, due to propagation of concentration disturbance with infinite diffusion speed in the Cahn-Hilliard's system. Hyperbolic system has a delay described by the second time derivative in Eqs (32) and (34). As a result, concentration disturbance in the hyperbolic system propagates with the finite speed and the instability realizes with the delay relatively to the Cahn-Hilliard's system. 


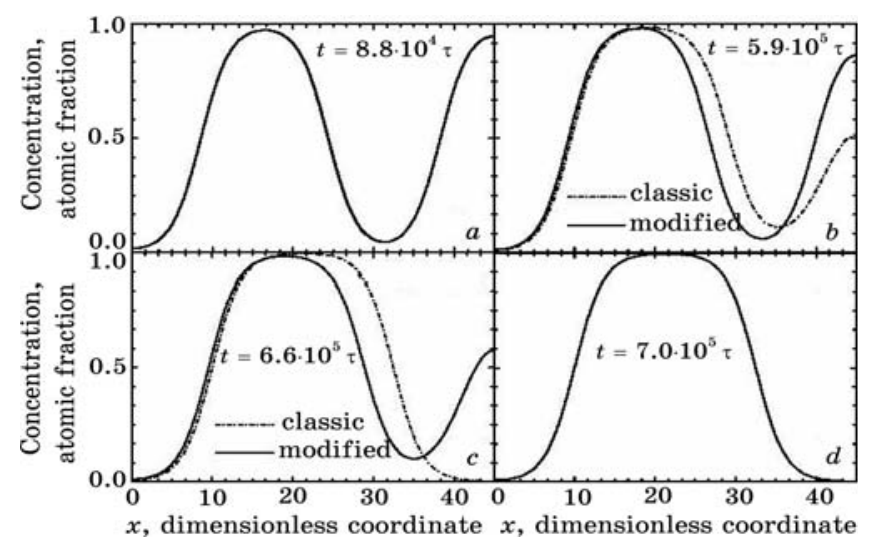

Fig. 6. Dynamics of spinodal decomposition of a binary liquid in $1 D$ case with the constant mobility $M=M_{0}=$ const: $(a)$ metastable state; $(b)$ beginning of the transition from unstable to metastable state; $(c)$ finishing of the transition from unstable to metastable state; $(d)$ new metastable state. The first metastable state $(a)$ and the following metastable state $(d)$ are equivalent for both classic and modified Cahn-Hilliard equations. The dynamics of transition between two metastable states is much more faster for classic Cahn-Hilliard equation.

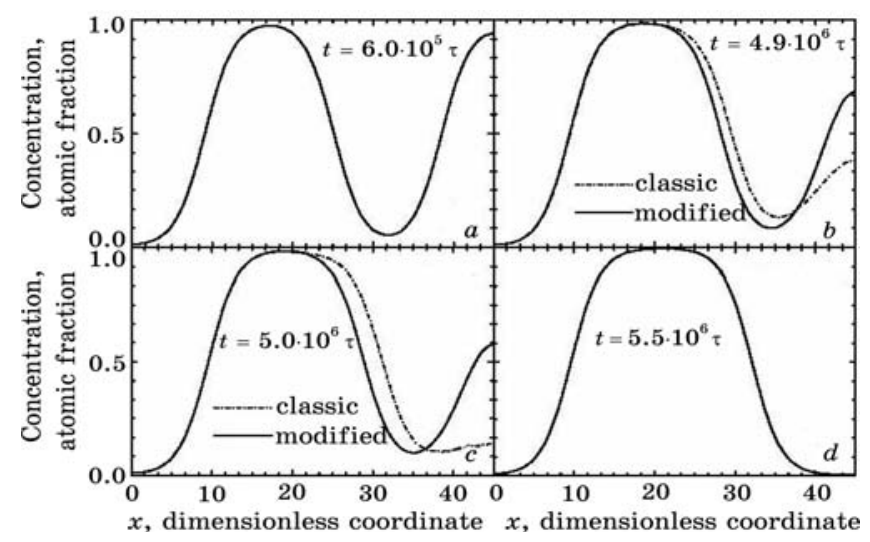

Fig. 7. Dynamics of spinodal decomposition of a binary liquid in $1 D$ case with the concentration dependence of mobility $M=M_{0} c(1-c):(a)$ metastable state; $(b)$ beginning of the transition from unstable to metastable state; $(c)$ transient period for the transition from unstable to metastable state; $(d)$ new metastable state. The first metastable state $(a)$ and the following metastable state $(d)$ are equivalent for both classic and modified Cahn-Hilliard equations. The dynamics of transition between two metastable states is much more faster for classic Cahn-Hilliard equation.

Finally, the new stable (or metastable) profile is formed which is the same for both parabolic Cahn-Hilliard's system and hyperbolic system. 
TABLE 3. Parameters for a binary system used in numeric computations.

\begin{tabular}{l|c|c}
\hline \multicolumn{1}{c|}{ Parameter } & $\begin{array}{c}\text { Value and unit for } \\
1 D \text { modelling }\end{array}$ & $\begin{array}{c}\text { Value and unit for } \\
3 D \text { modelling }\end{array}$ \\
\hline Initial concentration, $c_{0}$ & 0.5 atomic fraction & 0.5 atomic fraction \\
\hline Height of the free energy, $f_{0}$ & 0.5 & 0.5 \\
\hline Time for diffusion relaxation, $\tau_{D}$ & $1.5 \cdot 10^{-11} \mathrm{~s}$ & $1.5 \cdot 10^{-11} \mathrm{~s}$ \\
\hline Diffusion coefficient, $D$ & $5.0 \cdot 10^{-9} \mathrm{~m}^{2} / \mathrm{s}$ & $5.0 \cdot 10^{-9} \mathrm{~m}^{2} / \mathrm{s}$ \\
\hline Bulk diffusion speed, $V_{D}=\left(D / \tau_{D}\right)^{1 / 2}$ & $18.26 \mathrm{~m} / \mathrm{s}$ & $18.26 \mathrm{~m} / \mathrm{s}$ \\
\hline Spatial diffusion length, $l_{D}=D / V_{D}$ & $0.27 \cdot 10^{-9} \mathrm{~m}$ & $0.27 \cdot 10^{-9} \mathrm{~m}$ \\
\hline Ratio $r_{c} / l_{D}$ & 0.90 & 0.29 \\
\hline Quantity of computational nodes, $N$ & 80 & $500^{3}$ \\
\hline Dimensionless spatial step, $\Delta x$ & 0.56 & 0.88 \\
\hline Dimensionless time step, $\tau$ & $5.13 \cdot 10^{-2}$ & $5.0 \cdot 10^{-3}$ \\
\hline
\end{tabular}

The described dynamical scenario qualitatively does not depend on the atomic mobility of decomposing phases. Comparative dynamics shown in Figs. 6 and 7 for constant mobility and concentration dependent mobility, respectively, demonstrates that the both dynamics qualitatively remain the same. The only difference in dynamics shown in Figs. 6 and 7 is that the transition from the one metastable state to the next metastable state proceeds during various periods of time. Consequently, the Cahn and Hilliard model predicts much more faster dynamics of spinodal decomposition, which can be resulted in much more diffuse boundaries between two separated phases.

\subsection{D Modelling}

For $3 D$ numeric solution of Eqs (68)-(71), a cube with linear size $N$ is taken. The cube is approximated by the numerical grid with equal distances $\Delta x$ between nodes along Cartesian axes. In such a case, coordinates of the nodes are given as $x=i \Delta x, y=i \Delta x$, and $z=i \Delta x$, where $i=1, \ldots, N, j=1, \ldots, N$, and $k=1, \ldots, N$, respectively.

A random distribution around average concentration $c_{0}$ within the cube is accepted for initial time step $n=0$. Then for every time step $t=n \tau$, the following explicit numerical scheme is used:

$$
\left(\frac{\delta F}{\delta c}\right)_{i j k}^{n}=-\frac{f_{0}}{2}\left(1-c_{i j k}^{n}\right)\left(1-2 c_{i j k}^{n}\right) c_{i j k}^{n}+
$$




$$
\begin{gathered}
+\left(\frac{r_{c}}{l_{D} \Delta x}\right)^{2}\left(c_{i+1 j k}^{n}+c_{i-1 j k}^{n}+c_{i j+1 k}^{n}+c_{i j-1 k}^{n}+c_{i j k+1}^{n}+c_{i j k-1}^{n}-6 c_{i j k}^{n}\right), \\
J_{i j k}^{n+1}=(1-\tau) J_{i j k}^{n}+\frac{\tau}{2 \Delta x}\left(\left(\frac{\delta F}{\delta c}\right)_{i+1 j k}^{n}-\left(\frac{\delta F}{\delta c}\right)_{i-1 j k}^{n}+\right. \\
+\left(\frac{\delta F}{\delta c}\right)_{i j+1 k}^{n}-\left(\frac{\delta F}{\delta c}\right)_{i j-1 k}^{n}+\left(\frac{\delta F}{\delta c}\right)_{i j k+1}^{n}-\left(\frac{\delta F}{\delta c}\right)_{i j k-1}^{n}, \\
c_{i j k}^{n+1}=c_{i j k}^{n}-\frac{\tau}{2 \Delta x}\left(J_{i+1 j k}^{n}-J_{i-1 j k}^{n}+J_{i j+1 k}^{n}-J_{i j-1 k}^{n}+J_{i j k+1}^{n}-J_{i j k-1}^{n}\right) .
\end{gathered}
$$

A set of hyperbolic equations (78)-(80) allows to model decomposition with conserved function of concentration $c$. It has been resolved numerically with material and computational parameters summarized in Table 3. To test conservation of $c$, average concentration of a whole

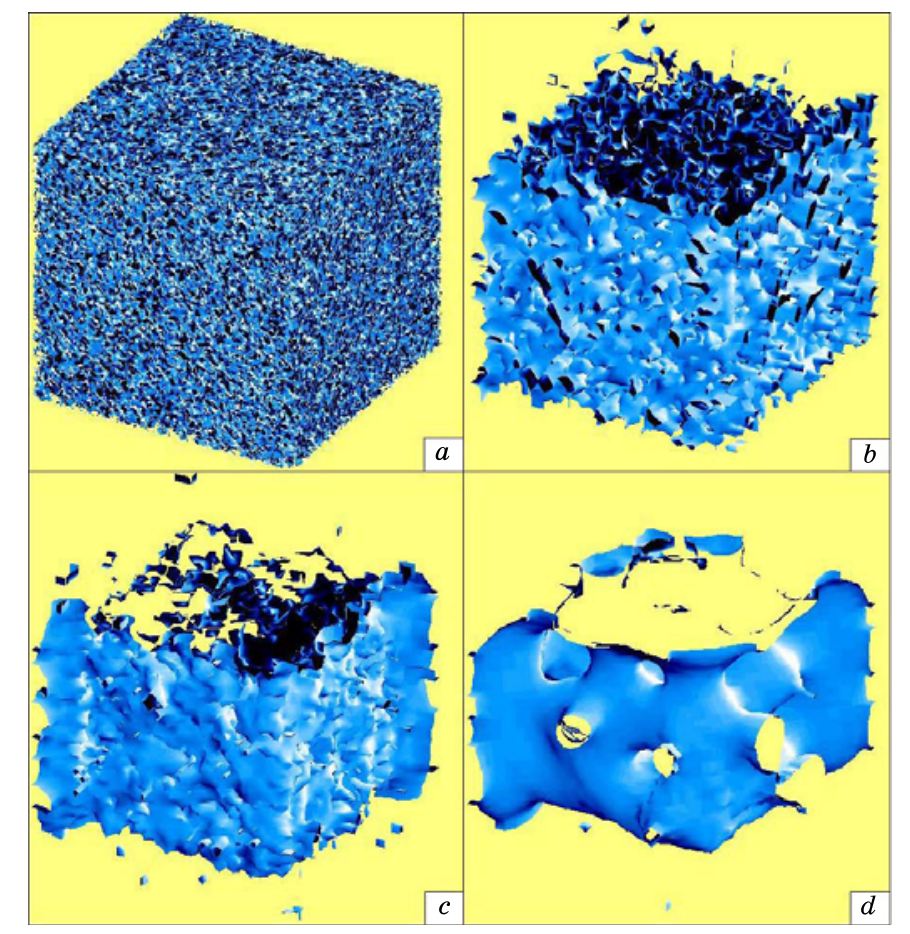

Fig. 8. $3 D$ modelling of spinodal decomposition in undercooled binary liquid: (a) $t=0$, (b) $t=150 \tau$, (c) $t=1.5 \cdot 10^{3} \tau$, (d) $t=1.5 \cdot 10^{4} \tau$. For every time moment, isoconcentration patterns within the cube with size of $500^{3}$ computational nodes is shown. 
computational domain has been compared with the initial value $c_{0}$. An error of computations $\left|c-c_{0}\right| / c_{0} \cdot \mathbf{1 0 0} \%$ was found not higher than $3.8 \%$. That confirms correctness of the present system $(78)-(80)$ to model the spinodal decomposition with conserved concentration $c$ within computational domain.

Figure 8 shows evolution of concentration inside the cube. It is seen that initially random parts of distribution with equal concentration (Fig. 8, $a$ ) create isoconcentration surface (Fig. 8, $b-d$ ] during decomposition. Figure 9 presents snapshots of patterns evolving in local nonequilibrium spinodal decomposition. This sequence exhibits hyperbolic evolution with a sharp boundary between two types of decomposed liquid especially at the first moments of decomposition (see Fig. 9, $b$ ). The sharp boundary between two liquids follows from the fact that description of diffusion in system (78)-(80) is given by hyperbolic type of
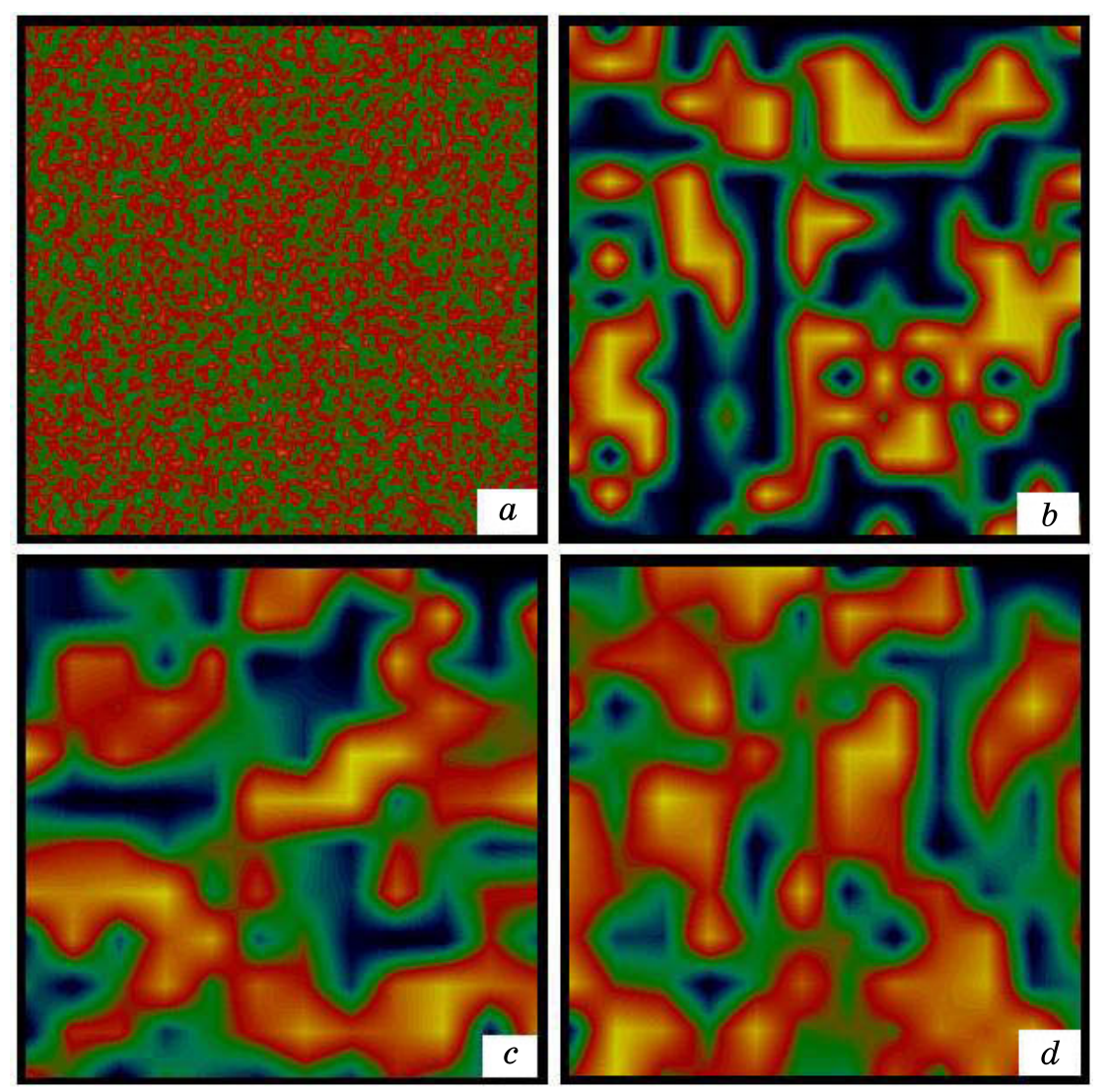

Fig. 9. Evolution of spinodal decomposition: (a) $t=0,(b) t=50 \tau,(c) t=10^{3} \tau$, (d) $t=3 \cdot 10^{4} \tau$. For every time moment, cross section for a cube with the size of $500^{3}$ computational nodes is shown. 
equation (69). Together with conservation law (70), Eq. (69) predicts with a finite speed and, as a consequence, with sharp diffusion fronts between two separating phases.

To compare patterns originating in spinodal decomposition, results for both hyperbolic evolution and parabolic evolution were extracted from solution of Eqs (78)-(80). Predictions of complete system (78)(80) were taken as for hyperbolic evolution. Predictions of the system (78)-(80) without relaxation of the solute diffusion flux, i.e., with the Fick's diffusion flux

$$
\mathbf{J}=\nabla\left(\frac{\delta F}{\delta c}\right)
$$

and its numerical approximation

$$
\begin{gathered}
J_{i j k}^{n}=\frac{1}{2 \Delta x}\left(\left(\frac{\delta F}{\delta c}\right)_{i+1 j k}^{n}-\left(\frac{\delta F}{\delta c}\right)_{i-1 j k}^{n}+\right. \\
+\left(\frac{\delta F}{\delta c}\right)_{i j+1 k}^{n}-\left(\frac{\delta F}{\delta c}\right)_{i j-1 k}^{n}+\left(\frac{\delta F}{\delta c}\right)_{i j k+1}^{n}-\left(\frac{\delta F}{\delta c}\right)_{i j k-1}^{n},
\end{gathered}
$$

instead of Eqs (69) and (79), respectively, were taken as for parabolic evolution.

Results of modelling for patterns in both evolutions are shown in Fig. 10. Evolution of patterns has been spied upon the complete sepa-

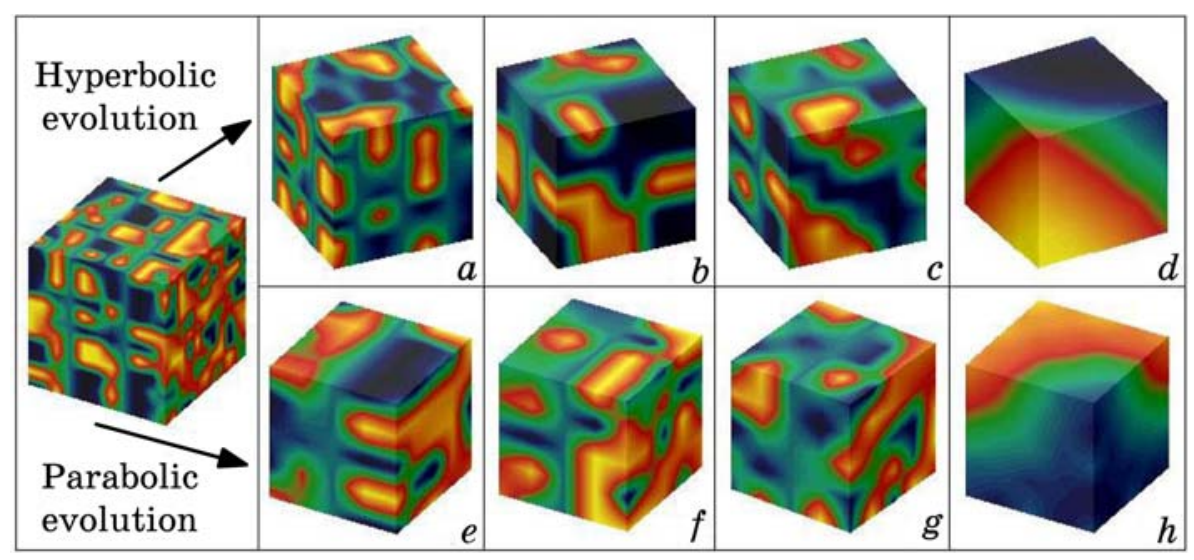

Fig. 10. Comparison of patterns in spinodal decomposition described by $(a-d)$ hyperbolic equation and $(e-h)$ parabolic equation. Here, $(a, e) t=10 \tau,(b, f)$ $t=40 \tau,(c, g) t=2 \cdot 10^{2} \tau,(d, h) t=2 \cdot 10^{5} \tau$. For every time moment, patterns are shown for a small cube of $50^{3}$ computational nodes extracted from central part of the cube with the size of $500^{3}$ computational nodes. 
ration of liquids (see Fig. 10, $d$ and $h$ ). It is seen that the boundaries between two demixing liquid phases are sharper for hyperbolic evolution than for the parabolic evolution. This feature of the hyperbolic evolution is a result of solute diffusion with the finite speed that leads to instability realized with the delay relatively to the Cahn-Hilliard's system (see comparative dynamics in Figs. 6 and 7).

\section{STOCHASTIC MODELS OF SPINODAL DECOMPOSITION}

In this Section, we consider to above models for spinodal decomposition and illustrate in what a manner concentration fluctuations, considered as internal noise, obeying fluctuation-dissipation relation can affect on the system dynamics and stationary states.

It is known that contrary to the naive predictions, assuming that fluctuations lead to disordering effects, phenomena such as noiseinduced transitions in zero-dimensional systems [60-64] (when a stochastic variable $x$ is a function of the time $t$ only, $x=x(t)$ ), stochastic resonance $[65,66]$, noise-induced ordered and disordered phase transitions in extended systems [67-70] (when $x=x(\mathbf{r}, t)$ ), noise-induced pattern formation processes [71, 72], noise-induced effects in excitable systems [73], and a lot of others are manifestations of the constructive role of fluctuating environment. An increasing interest in the noise-induced phenomena in extended systems results in the discovery of new nonequilibrium universality classes $[74,75]$ and new types of self-organization processes such as entropy driven phase transitions [76, 77].

In most problems of noise-induced phenomena in extended systems external fluctuating sources are considered; their primary role in selforganizational processes is stated [67, 78]. Recently, a new type of entropy driven phase transitions was discovered [76]. Within this type of transitions, it was shown that internal fluctuations with intensity related to a field-dependent kinetic coefficient (mobility) play a principle role in ordering dynamics. Particularly, it was found that the internal multiplicative noise leads to the effective entropy dependence on the stochastic quantity in a functional form but does not change the corresponding free energy functional. As a result, noise-induced effects can be understood with a help of the entropy mechanism, which follows from the thermodynamics. Considering parabolic and hyperbolic models for spinodal decomposition within the frame of the linear stability analysis and the mean field theory we compare behaviour of above two models.

The aim of this Section is to perform a somewhat detailed study of entropy driven phase transitions mechanisms in phase separation processes. We analyze early and late stages of evolution numerically. For the stationary picture, we extend the mean field approach to the systems with the field dependent mobility and investigate mechanisms 
of entropy driven phase transitions in binary stochastic systems. We generalize the well-known results for early stages of evolution, study late stages and consider re-entrant ordering effects in a stationary case. Our analytical results are compared with computer simulations.

The outline of this Section is the following. In Subsection 4.1, we introduce the general stochastic system with conserved dynamics and obtain the corresponding Fokker-Planck equation that can be used in the mean field analysis for both parabolic and hyperbolic models. Considering parabolic model, we discuss effects of the internal multiplicative noise influence at early and late stages of spinodal decomposition and consider phase transitions by means of the mean field theory. In Subsection 4.1.4, we discuss effect of a combined effect of both internal and external stochastic sources. In Subsection 4.2, we study stochasticity of the hyperbolic transport. Subsection 4.3 is devoted to study of stochastic hyperbolic model for spinodal decomposition.

\subsection{Stochastic Parabolic Model for Spinodal Decomposition}

In this subsection, we investigate an influence of the conserved-field (concentration) dependent mobility and the corresponding internal noise on properties of a phase separation scenario in the parabolic model.

Formally, in a case of a binary system with concentrations $\rho_{A}$ and $\rho_{B}$ of the components $A$ and $B$, respectively, the density difference $x=\rho_{A}-\rho_{B}$ can be introduced. In a phase separation scenario, the quantity $x$ obeys a conservation law $\int x(\mathbf{r}, t) \mathrm{d} \mathbf{r}=$ const . In our study, we use the field $x$ to describe the system under consideration and investigate a corresponding dynamics and a stationary picture.

To define a principle model let us start with a continuity equation for the field $x(\mathbf{r}, t)$ in a $d$-dimensional space in the form

$$
\frac{\partial}{\partial t} x=-\nabla \cdot \mathbf{J}
$$

where $J$ is the flux. The deterministic part of the flux is of the form

$$
\mathbf{J}_{\text {det }}=-M \nabla \frac{\delta \mathscr{F}[x]}{\delta x} \text {. }
$$

Here, $M$ is the mobility, the Helmholtz coarse-grained free energy functional $\mp$ is of the standard (Ginzburg-Landau or Cahn-Hilliard) form applicable for a local equilibrium system

$$
\mathscr{F}[x]=\int d V\left\{f(x)+\frac{D}{2}(\nabla x)^{2}\right\},
$$


where $D$ is the spatial coupling intensity related to the correlation radius in the form $D=r_{c}^{2}$ with $r_{c}^{2}=\left.\left(\delta^{2} \mathscr{F} / \delta(\nabla x)^{2}\right)\right|_{\nabla x=0} ; f(x)$ is the free energy density of the bulk. The mobility we assume in a functional form, i.e. $M=M(x)$.

To investigate the influence of the flux fluctuations, let us introduce fluctuation source $\zeta$ into the right hand side of Eq. (84). It yields an expression for the flux in the form as follows:

$$
\mathbf{J}=\mathbf{J}_{\mathrm{det}}+\zeta(x ; \mathbf{r}, t) .
$$

Formally, the stochastic part $\zeta$ is assumed to be Gaussian, and generally, it can be a function of the field $x$. Assuming the $x$-dependent mobility $M=M(x)$ and using fluctuation dissipation relation, one gets the following definition for the averages

$$
\langle\zeta(x ; \mathbf{r}, t)\rangle=0, \quad\left\langle\zeta(x ; \mathbf{r}, t) \zeta\left(x ; \mathbf{r}^{\prime}, t^{\prime}\right)\right\rangle=2 \sigma^{2} M(x) C\left(\mathbf{r}-\mathbf{r}^{\prime} ; t-t^{\prime}\right) .
$$

In the simplest case, we assume the correlation function $C$ in the form of $C\left(\mathbf{r}-\mathbf{r}^{\prime} ; t-t^{\prime}\right) \rightarrow \delta\left(\mathbf{r}-\mathbf{r}^{\prime}\right) \delta\left(t-t^{\prime}\right)$, which allows to consider the white noise in space and time. Here, we note that the fluctuation dissipation relation holds that yields an interpretation of the noise intensity $\sigma^{2}$ as an effective temperature of the bath.

In further study of stochastic dynamics, we consider a general case when control parameters of the system and the noise intensity $\sigma^{2}$ are independent quantities.

Using conditions corresponding to an equilibrium situation, one gets the flux $J$ as follows $\mathbf{J}=-M \nabla \delta \mathscr{F}[x] / \delta x+g(x) \xi(\mathbf{r}, t)$, where $g(x)=\sqrt{M(x)}, \quad\langle\xi(\mathbf{r}, t)\rangle=0, \quad\left\langle\xi(\mathbf{r}, t) \xi\left(\mathbf{r}^{\prime}, t^{\prime}\right)\right\rangle=2 \sigma^{2} \delta\left(\mathbf{r}-\mathbf{r}^{\prime}\right) \delta\left(t-t^{\prime}\right)$. Substituting the generalized flux into Eq. (83), we get the stochastic continuity equation in the form

$$
\frac{\partial}{\partial t} x=\nabla \cdot\left(M(x) \nabla \frac{\delta \mathscr{F}[x]}{\delta x}\right)+\nabla g(x) \xi(\mathbf{r}, t) .
$$

To study statistical properties of the system one needs to find the probability density $\mathcal{P}=\mathcal{P}([x], t)$. To this end, we represent the system on a regular $d$-dimension lattice with a mesh size $\ell$. Then, the partial differential equation (88) is reduced to a set of usual differential equations written for an every cell $i$ on a grid in the form

$$
\frac{d x_{i}}{d t}=\left(\nabla_{L}\right)_{i j} M_{j}\left(\nabla_{R}\right)_{j l} \frac{\partial F}{\partial x_{l}}+\left(\nabla_{L}\right)_{i j} g_{j} \xi_{j}(t),
$$

where index $i$ labels cells: $i=1, \ldots, N^{d}$; the discrete left and right operators are introduced ( $d=2)$ as follows: 


$$
\begin{aligned}
& \left(\nabla_{L}\right)_{i j}=\frac{1}{\ell}\left(\delta_{i, j}-\delta_{i-1, j}\right), \quad\left(\nabla_{R}\right)_{i j}=\frac{1}{\ell}\left(\delta_{i+1, j}-\delta_{i, j}\right), \\
& \left(\nabla_{L}\right)_{i j}=-\left(\nabla_{R}\right)_{j i}, \quad\left(\nabla_{L}\right)_{i j}\left(\nabla_{R}\right)_{j l}=\Delta_{i l}=\frac{1}{\ell^{2}}\left(\delta_{i, l+1}-2 \delta_{i, l}+\delta_{i, l-1}\right),
\end{aligned}
$$

where $\delta_{i j}$ is the Kronecker symbol. For stochastic sources, the discrete correlator is of the form $\left\langle\xi_{i}(t) \xi_{j}(t)\right\rangle=2 \ell^{-2} \sigma^{2} \delta_{i j} \delta\left(t-t^{\prime}\right)$. In the following analysis, we use the Stratonovich interpretation of the Langevin equations (89).

Next, to obtain above distribution let us introduce standard definitions

$$
\mathcal{P}([x], t)=\left\langle\overline{\prod_{i=1}^{N^{d}} \delta\left(x_{i}(t)-x_{i}\right)}\right\rangle \equiv\langle\bar{\rho}(t)\rangle,
$$

where $\ldots$ and $\langle\ldots\rangle$ are averages over initial conditions and noise, respectively. To obtain the corresponding Fokker-Planck equation, we use the standard technique and exploit the stochastic Liouville equation

$$
\frac{\partial}{\partial t} \bar{\rho}=-\sum_{i} \frac{\partial}{\partial x_{i}}\left(\dot{x}_{i} \bar{\rho}\right)
$$

Inserting the expression for the time derivative from Eq. (89) and averaging over noise, we get

$$
\frac{\partial}{\partial t}\langle\bar{\rho}\rangle=-\sum_{i} \frac{\partial}{\partial x_{i}}\left(\left(\nabla_{L}\right)_{i j} M_{j}\left(\nabla_{R}\right)_{j l} \frac{\partial F}{\partial x_{l}}\right)\langle\bar{\rho}\rangle-\sum_{i j} \frac{\partial}{\partial x_{i}}\left(\nabla_{L}\right)_{i j}\left\langle g_{j} \xi_{j}(t) \bar{\rho}\right\rangle .
$$

The correlator in the second term can be calculated by means of Novikov's theorem that gives [79]

$$
\left\langle g_{j} \xi_{j}(t) \bar{\rho}\right\rangle=\sum_{k} 2 \sigma^{2} \int_{0}^{t} d t^{\prime} \delta_{j k} \delta\left(t-t^{\prime}\right)\left\langle\frac{\delta g_{j} \bar{\rho}(t)}{\delta \xi_{k}\left(t^{\prime}\right)}\right\rangle,
$$

where for the last multiplier one has

$$
\frac{\delta g_{j} \bar{\rho}(t)}{\delta \xi_{k}\left(t^{\prime}\right)}=-\left.g_{j} \frac{\partial}{\partial x_{l}} \frac{\delta x_{l}(t)}{\delta \xi_{k}\left(t^{\prime}\right)}\right|_{t=t^{\prime}} \bar{\rho}(t) .
$$

In a formal solution of the Langevin equation, the response function takes the form

$$
\left.\frac{\delta x_{l}(t)}{\delta \xi_{k}\left(t^{\prime}\right)}\right|_{t=t^{\prime}}=\left(\nabla_{L}\right)_{l k} g_{k}
$$


After some algebra, we obtain the Fokker-Planck equation for the total distribution $\mathcal{P}$ in the discrete space

$$
\frac{\partial}{\partial t} \mathcal{P}=-\sum_{i} \frac{\partial}{\partial x_{i}}\left(\left(\nabla_{L}\right)_{i j} M_{j}\left(\nabla_{R}\right)_{j l} \frac{\partial F}{\partial x_{l}}\right) \mathcal{P}-\sigma^{2} \sum_{i j} \frac{\partial}{\partial x_{i}}\left(\nabla_{L}\right)_{i j} g_{j} \frac{\partial}{\partial x_{j}}\left(\nabla_{R}\right)_{j i} g_{i} \mathcal{P},
$$

where relations between left and right gradient operators are used. The one-point probability density is defined as follows:

$$
P_{i}(t)=\int \prod_{m \neq i} \mathcal{P}([x], t) d x_{m} .
$$

The above equation can be rewritten in the standard form (in our case, it has only formal form) for the functional $\mathcal{P}=\mathcal{P}(\{x(\mathbf{r})\}, t)$ in the continuum space as follows:

$$
\begin{gathered}
\frac{\partial}{\partial t} \mathcal{P}=-\int d \mathbf{r} \frac{\delta}{\delta x(\mathbf{r})}\left[\nabla M \nabla \frac{\delta \mp}{\delta x(\mathbf{r})}-\frac{\sigma^{2}}{2} \nabla\left(\nabla \frac{\delta M}{\delta x(\mathbf{r})}\right)\right] \mathcal{P}- \\
-\sigma^{2} \int d \mathbf{r} \frac{\delta^{2}}{\delta x^{2}(\mathbf{r})} \nabla(\nabla M) \mathcal{P} .
\end{gathered}
$$

The term proportional to $\sigma^{2}$ in the first addendum is the noise-induced drift. The derived Fokker-Planck equation allows us to write down the corresponding Langevin equation

$$
\frac{\partial}{\partial t} x(\mathbf{r}, t)=\nabla M \nabla \frac{\delta \mathscr{F}}{\delta x}-\frac{\sigma^{2}}{2} \nabla\left(\nabla \frac{\delta M}{\delta x}\right)+\nabla g \xi_{W}(\mathbf{r}, t),
$$

with a process $\xi_{W}$, which has a strong mathematical definition through the Wiener process $W(t): \xi_{W}(t)=d W(t) / d t,(d W)^{2}: d t[60]$.

The stationary solution of the Fokker-Planck equation takes the form

$$
\mathcal{P}[x] \propto \exp \left\{-\frac{1}{\sigma^{2}}\left(\mathscr{F}[x]+\frac{\sigma^{2}}{2} \int d \mathbf{r} \ln M(x)\right)\right\} .
$$

Exploiting standard thermodynamic definition of the effective internal energy $\mathcal{U}_{\text {eff }}=\mathscr{F}+T_{\text {ef }} \oint_{\text {eff }}$ and assuming a quasi-Gibbs form for the stationary distribution, we can identify the effective entropy

$$
S_{\text {eff }}=(1 / 2) \int d \mathbf{r} \ln M(x)
$$

and the effective temperature $T_{\text {ef }}=\sigma^{2}$.

It is principally important that the stationary distribution is exact and is described not only by the initial functional $\mathscr{F}[x]$. Here, the entropy contribution modifies the form of the probability density. Despite, we consider the internal multiplicative noise, its action leads to 
the entropy variability. Thus, controlling the noise intensity or parameters in the mobility $M(x)$ we can govern an ordering process. Therefore, the system studied below is considered as a nonequilibrium one. The same situation is observed in the entropy driven phase transitions in systems with nonconserved dynamics [76, 80-82], where the stationary distribution is obtained exactly and phase transitions picture is controlled by the effective entropy variations. Below, we relate the entropy driven phase transitions formalism developed for systems with nonconserved dynamics to the systems with conserved dynamics. The main attention will be paid on effects where the entropy variability is principle.

Using Langevin or Fokker-Planck equation, one can derive equation for the first statistical moment directly:

$$
\frac{\partial}{\partial t}\langle x(\mathbf{r}, t)\rangle=\nabla\left\langle M \nabla \frac{\delta \mathscr{F}}{\delta x(\mathbf{r})}\right\rangle-\frac{\sigma^{2}}{2} \nabla\left\langle\nabla \frac{\delta M}{\delta x(\mathbf{r})}\right\rangle .
$$

This equation can be used to analyze the influence of the internal multiplicative noise on the stability of the null phase in the linear approximation.

Since the dynamics is conserved,

$$
\int_{\mathrm{V}} x(\mathbf{r}, t) d \mathbf{r}=\text { const },
$$

$V$ is the system volume), so dynamics of the phase separation can be considered with a help of the Fourier transform of correlation function

$$
G(\mathbf{r}, t)=(1 / V) \int\left\langle x\left(\mathbf{r}^{\prime}, t\right) x\left(\mathbf{r}^{\prime}+\mathbf{r}, t\right)\right\rangle d \mathbf{r}^{\prime}-\langle x(\mathbf{r}, t)\rangle^{2} .
$$

The corresponding structure function is given as

$$
S(\mathbf{k}, t)=\int_{\mathrm{V}} G(\mathbf{r}, t) e^{i \mathbf{k r}} d \mathbf{r} .
$$

In practice, it is convenient to use a spherical average of the correlation and structure functions, which are as follows:

$$
g(r, t)=\int_{\Omega_{r}} G(\mathbf{r}, t) d \Omega, \quad S(k, t)=\int_{\Omega_{k}} S(\mathbf{k}, t) d \Omega .
$$

Here, $\Omega_{r}$ and $\Omega_{k}$ are spherical shells of radius $r$ and $k$, respectively. The above values allow us to extract a mean characteristic size of domains at time $t, R(t)$, using scaling relations: $g(r, t)=\varphi(r / R(t))$, $S(k, t)=R^{d}(t) \varphi(k R(t))$. The expected domains growth law is $R(t) \propto t^{z}$, where $z$ is the domain-growth exponent.

The Model. In our consideration, we use a model for a binary system, which is described by the free-energy density, $f(x)$. Single-phase equilibrium $f(x)$ has a stable single-well structure. In a two-phase region, $f(x)$ is of a double-well structure; the corresponding model has the form 


$$
f(x)=-\varepsilon x^{2} / 2+x^{4} / 4,
$$

where $\varepsilon$ is a dimensionless phenomenological constant, playing the role of a control parameter.

The mobility is used in the functional form

$$
M(x)=\left(1+\alpha x^{2}\right)^{-1}, \quad \alpha \geq 0 .
$$

Variations in the parameter $\alpha$ allow us to consider additive $(\alpha=0)$ or multiplicative ( $\alpha \neq 0)$ noises, separately (the difference in such a classification is reduced to the following: if the noise term appears in the evolution equation with a constant multiplier, $g=$ const, then the noise is additive, else, $g=g(x)$, it is multiplicative). The model function (103) assumes that fluctuations are large in the case where $x=0$, whereas fluctuations are small in cases where $x \neq 0$. Formally, assuming $\alpha$ to be small, an approximate definition is $M(x) \approx 1-\alpha x^{2}$. As previous studies show, the quantity $\alpha$ can be expressed through the relation between bulk $D_{b}$ and surface $D_{s}$ diffusion constants, i.e. $\alpha \approx 1-D_{b} / D_{s}$ (see Ref. [83]). Further, we are looking for changes in system behaviour when $\alpha$ ranges.

In such kind of stochastic models, a possible scenario of phase separation depends on the initial conditions: at $\langle x(\mathbf{r}, 0)\rangle=0$, the system evolves by spinodal decomposition scenario (see Fig. 11, $a$ ), whereas at $\langle x(\mathbf{r}, 0)\rangle \neq 0$, a nucleation process is realized. (Fig. 11, $b$ ).
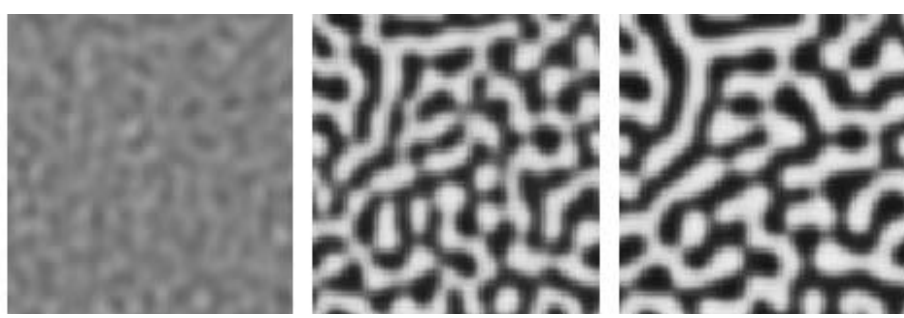

$a$
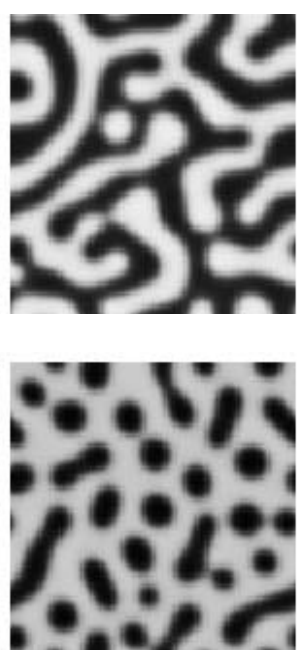

Fig. 11. Typical spatial patterns: $(a)$ spinodal decomposition $\langle x(\mathbf{r}, 0)\rangle=0,(b)$ nucleation $\langle x(\mathbf{r}, 0)\rangle=0.2$. Other parameters are as follows: $N \times N=120 \times 120$, $D=4, \varepsilon=1, \alpha=0.5, \sigma^{2}=0.2$. 


\subsubsection{An Early Stage of Evolution}

At the first, we investigate the internal multiplicative noise influence on instability of the homogeneous phase $\langle x\rangle=0$. Using Fourier transformation for the scalar field

$$
x_{\mathbf{k}}(t)=(2 \pi)^{-d} \int d \mathbf{r} x(\mathbf{r}, t) e^{-i \mathbf{k r}}
$$

in $d$-dimensional space, in linear approximation one gets

$$
\frac{d\left\langle x_{\mathbf{k}}\right\rangle}{d t}=-k^{2}\left(D k^{2}-\varepsilon+\alpha \sigma^{2}\right)\left\langle x_{\mathbf{k}}\right\rangle .
$$

It is principally important that the noise contribution denoted as $\alpha \sigma^{2}\left\langle x_{k}\right\rangle$ stabilizes the homogeneous state. The same result was observed in the case of entropy-driven phase transitions with nonconserved dynamics [76, 81]: as it follows, one can await the similar behaviour of the stochastic systems with conserved dynamics where the entropy driven phase transition formalism can be generalized.

More information of the system behaviour provides the knowledge of the structure function $S(\mathbf{k}, t)=\left\langle x_{\mathbf{k}}(t) x_{-\mathbf{k}}(t)\right\rangle$. Following the standard approach, a linear evolution equation for the spherically averaged structure function can be derived in the form [84]

$$
\frac{d S(k, t)}{d t}=-k^{2}\left(D k^{2}-\varepsilon+\alpha \sigma^{2}\right) S(k, t)+2 \sigma^{2} k^{2}-2 \alpha \sigma^{2} k^{2} \frac{1}{(2 \pi)^{d}} \int d \mathbf{q} S(q, t) .
$$

It is seen at $\alpha=0$ that corresponds to the additive noise case, one arrives at the well-known Cahn-Hilliard-Cook equation for the structure function [12, 59]. From exponential solutions of Eqs $(104,105)$, one can see that only modes with $k<k_{c}=\sqrt{\left(\varepsilon-\alpha \sigma^{2}\right) / D}$ are unstable and grow at early stages of evolution. With an increase in $\alpha$ or $\sigma^{2}$, the size of the unstable modes domain $k<k_{c}$ decreases. Modes with $k>k_{c}$ remain stable during the linear regime. Note that unstable modes cannot be realized at condition $\varepsilon<\alpha \sigma^{2}$. As it follows, the domain growth should be different for additive and multiplicative noise.

In Figure 12, we present solutions of the evolution equation (105) at different values of the parameter $\alpha$. It can be seen that an increase in $\alpha$ leads to a shift of the peak position toward smaller values of $k$. The peak of $S(k)$ is less pronounced in the multiplicative noise case than in the case of the additive noise. It follows that, if the multiplicative noise is considered, then the dynamic is slowed. A decrease in the peak height means that an interface is more diffuse in the case of multiplicative noise (see insertions in Fig. 12). We compare analytical results with computer simulations at the same time $t$ on the two-dimensional lattice. In the insertions, a typical patterns and images of spherically averaged 


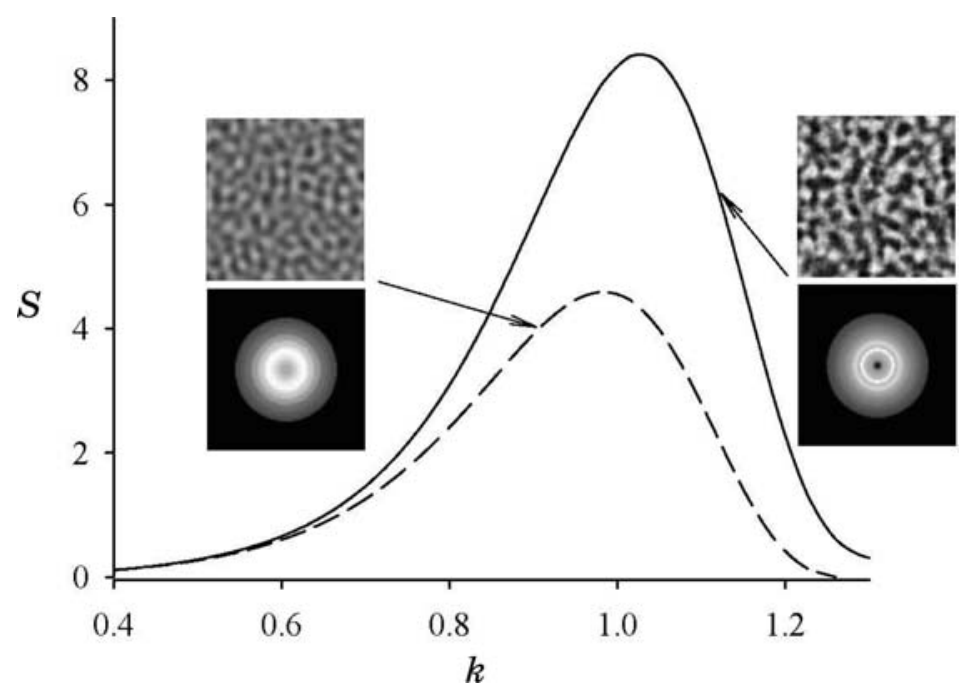

Fig. 12. Evolution of the structure function at early stage $(t=10)$ at $D=4$, $\varepsilon=1, \sigma^{2}=0.3$. Different values of the parameter $\alpha$ are used to compare influence of additive $\alpha=0$ and multiplicative $\alpha=0.9$ noises (solid and dashed lines, respectively). Insertion shows typical patterns and corresponded images of spherically averaged structure functions at the same time obtained from numerical solution of Eq. (99) at $x^{3}=0$ with the initial condition $\langle x(\mathbf{r}, 0)\rangle=0$.

structure functions are shown. It is seen that in the multiplicative noise case the pattern has more diffuse interface and the resonance ring in $S(k)$-dependence is less pronounced than at the additive noise.

\subsubsection{A Late Stage}

To get more information about internal multiplicative noise influence, we simulate the system behaviour and study influence of the parameter $\alpha$ at the late stage of the evolution. All simulations were done in twodimensional lattice with periodic boundary conditions with $N=120$. A criterion for the phase separation in the model under consideration is the growth of the averaged second moment of $x$ in the real space. We use the standard definition of the corresponding order parameter

$$
M_{2}(t)=N^{-2}\left\langle\sum_{i=1}^{N^{2}} x_{i}^{2}(t)\right\rangle
$$

An alternative formula is $M_{2}(t)=\sum_{k} S_{k}(t)$. In phase separation scenario during the long time evolution, the quantity $M_{2}(t)$ grows to the stationary value $M_{2}$ when the system tends to the nonzero stationary state. 

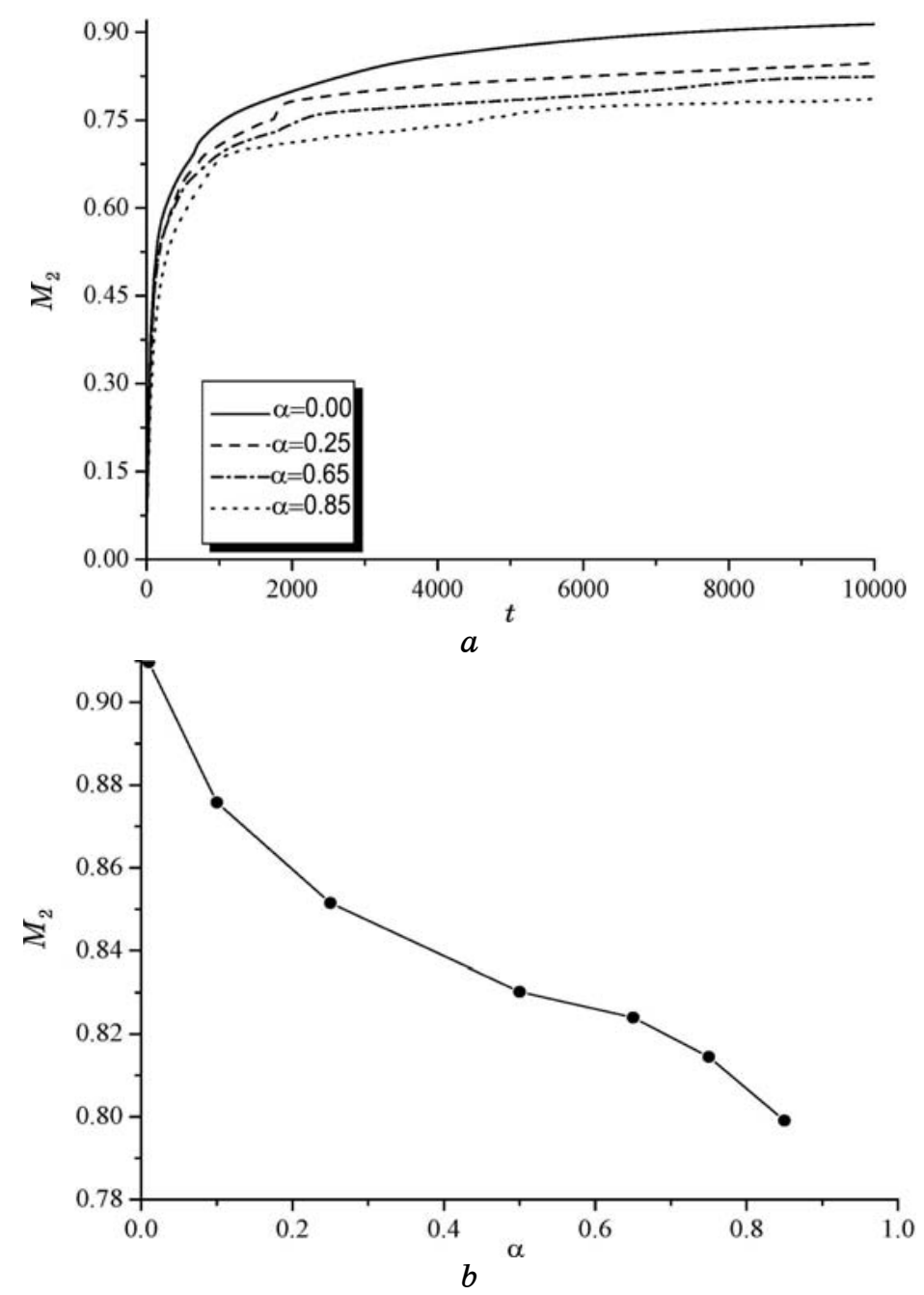

Fig. 13. Order parameter evolution $(a)$ and its stationary values $(b)$ at different values of $\alpha$ and $\varepsilon=1.0, D=4, \sigma^{2}=0.2$.

Figure 13 displays the evolution of the order parameter $M_{2}$ at different values of the parameter $\alpha$ and stationary values $M_{2}$ versus $\alpha$. It is seen that an increase in $\alpha$ delays slightly the evolution of $M_{2}$ (Fig. 13,a) at small times and suppresses the stationary values (see Fig. 13, $b$ ).

Despite the fact that the quantity $M_{2}$ represents an integral effect, more information about the system behaviour can be found in the structure function $S(k)$. A convenient quantity is the spherically averaged structure function defined on a circle as follows: 


$$
S(k, t)=\frac{1}{N_{k}} \sum_{k \leq \mathbf{k} \leq k+\Delta k} S(\mathbf{k}, t) .
$$

In Figure 14, we display the evolution of the structure function for two values of the parameter $\alpha$ at fixed noise intensity $\sigma^{2}$. Comparing Fig. $14, a, b$, one can see that, in the case under consideration, the peaks of the structure function are less pronounced when $\alpha$ increases that corresponds to the case of the field-dependent mobility case studied in Ref. [85] and relates to the linear stability analysis. As follows from Figs. 14, $a, b$, the positions of the peaks are the same with an increase in $\alpha$ at equal times. It follows from the linear stability analysis and corresponds to the fact that in the case of multiplicative noise the dynamics is slowed [85]. This result is different from the deterministic case where at large $\alpha$ peaks are located at higher values of $k$. The typical behaviour of the Fourier images in Fig. 14, $c$, shows the diffuse interface between two phases and change in the peaks position. As Figure
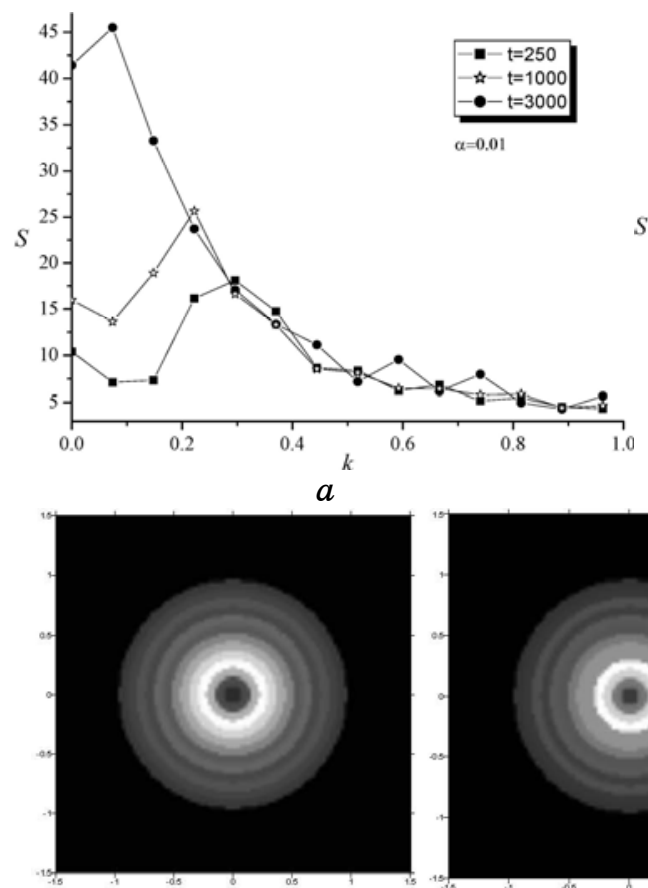

250

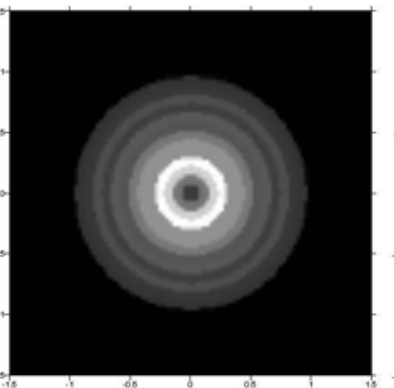

500

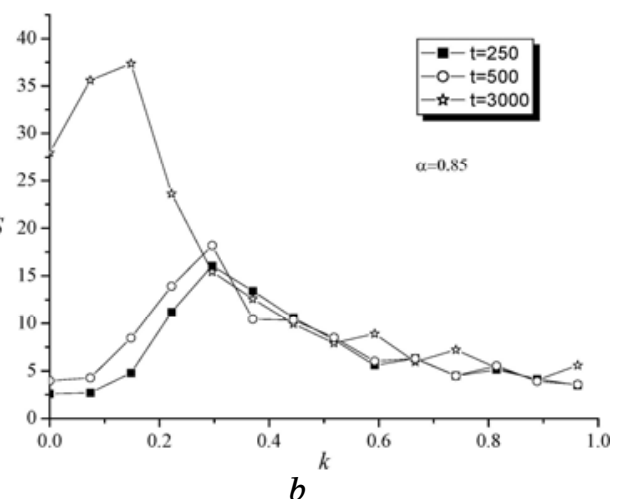

$b$

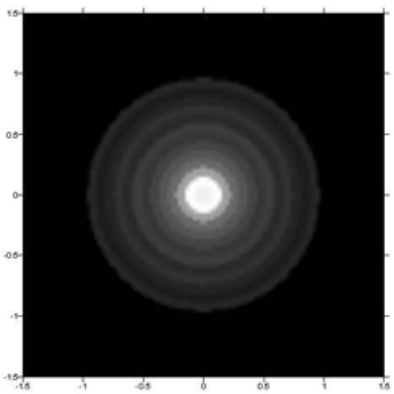

3000

Fig. 14. Evolution of the structure function at $\varepsilon=1.0, D=4.0, \sigma^{2}=0.2$ : (a) $\alpha=0.01$, (b) $\alpha=0.85$. The times represented are $t=250, t=1000$, $t=3000$. (c) Fourier images of structure function evolution at $\alpha=0.85$ and times $t=250, t=1000, t=3000$. 


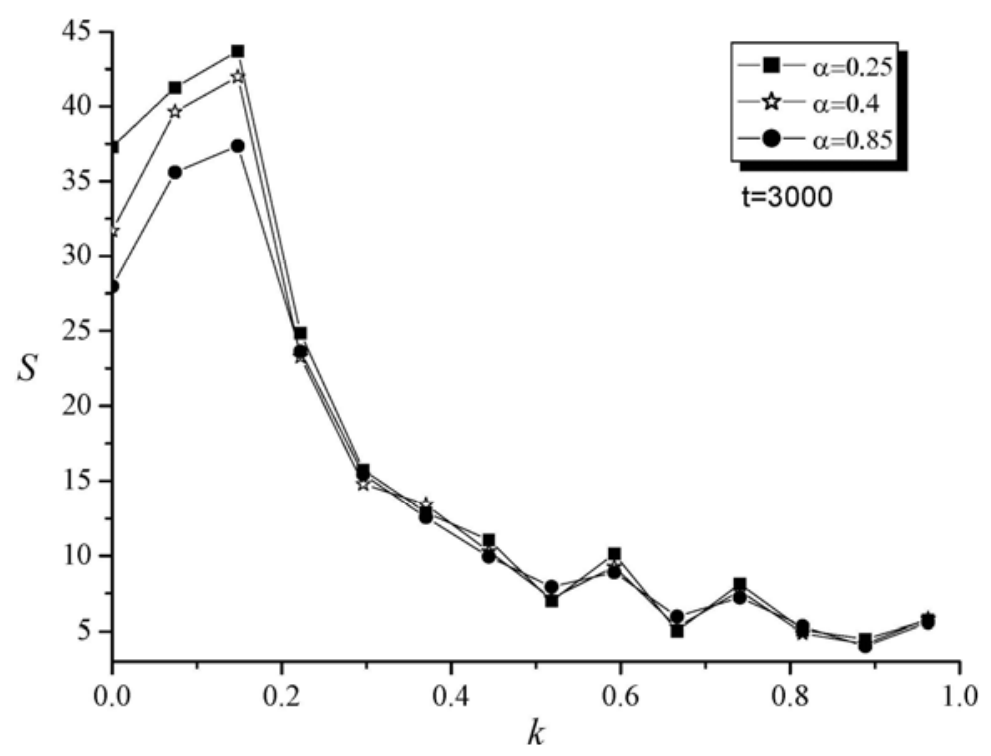

Fig. 15. Structure function behaviour at different values of the parameter $\alpha$ at $\varepsilon=1.0, \sigma^{2}=0.2, D=4.0, t=3000$.

15 shows, the change in peaks height means that with an increase in $\alpha$ the interface becomes more diffuse.

To investigate the domain growth dynamics we use the standard formulas for a relevant length:

$$
R(t)=\langle k\rangle^{-1}, \quad\langle k\rangle=\int_{0}^{k_{\max }} S(k, t) k d k / \int_{0}^{k_{\max }} S(k, t) d k .
$$

The power law behaviour of the function $R(t) \propto t^{z}$ is verified at different values of the parameter $\alpha$, where the domain growth exponent depends on $\alpha$, i.e. $z=z(\alpha)$ (see Fig. 16). It is seen that, in the case of additive noise ( $\alpha=0$ ), the exponent $z \propto 1 / 3$, whereas at $\alpha=1.0$, we obtain $z \propto 1 / 4$. Therefore, with an increase in $\alpha$ a crossover of dynamical regimes is observed. Our results are in good correspondence with deterministic and stochastic approaches, which indicate that an increase in the parameter $\alpha$ delays the dynamics [86-88].

\subsubsection{Stationary Case}

To investigate the steady states, we can use an extension of the mean field theory developed for the systems with conserved dynamics [37]. In the framework of this theory, one can use thermodynamic suppositions for the deterministic dynamics and after apply it to the stochastic one. 

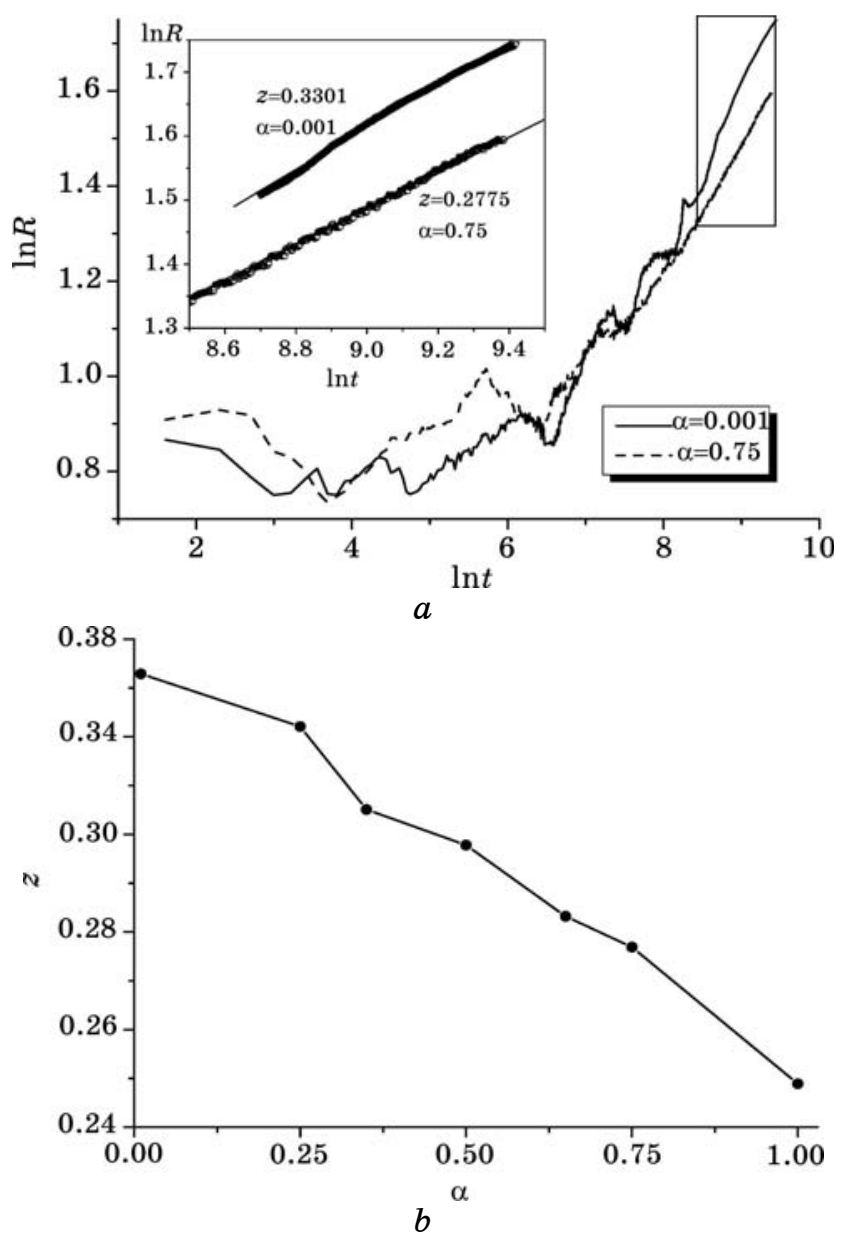

Fig. 16. Power law for domains size growth: $(a) \log -\log$ plot of the evolution of $R(t)$ at different values of the parameter $\alpha$ (insertion shows universal behaviour of the function $R(t)$ at large times indicated in the rectangle); $(b)$ dependence of the power law exponent $z$ versus parameter $\alpha$. Other parameters are as follows: $\varepsilon=1.0, D=4.0, \sigma^{2}=0.2$.

At first, let us define transition and critical points [89]. Considering the deterministic case, we use the model $\partial_{t} x=\nabla \cdot M \nabla \delta \mathscr{F} / \delta x$, where the restriction

$$
x_{0}=\int_{V} d \mathbf{r} x(\mathbf{r}, t)
$$

is taken into account, $x_{0}$ is fixed by the initial conditions. For such a system, the transition point is $\varepsilon_{T}\left(x_{0}\right)$ : at $\varepsilon<\varepsilon_{T}\left(x_{0}\right)$ the homogeneous state $x_{0}$ is stable; at $\varepsilon>\varepsilon_{T}\left(x_{0}\right)$ the system separates in bulk phases, $x_{1}$ 
and $x_{2}$, with $\langle x\rangle=x_{0}$. The transition point coincides with critical one for $x_{0}=0$ only, i.e. $\varepsilon_{T}(0)=\varepsilon_{c}$.

The corresponding steady state solutions are given as solutions of the equation $\nabla \cdot M \nabla \delta \mathscr{F} / \delta x=0$. If no flux condition is applied, then stationary solutions can be obtained from the equation $\nabla^{2} \delta \mathscr{f} / \delta x=0$, due to the mobility $M$ does not affect on the number and extremes positions of the functional $\mathscr{F}$; the mobility leads to the change in the dynamics of the phase transition only. Hence, the bounded solution is $\delta \mp / \delta x=h$, where $h$ is a constant effective field of the system, in equilibrium systems $h$ is a chemical potential. In the homogeneous case, the value $h$ does depend on the initial conditions $x_{0}$. Above the transition point, the steady state is not globally homogeneous, here the system separates into two bulk phases with values $x_{1}$ and $x_{2}$. The fraction $u$ of the system can be defined by the lever rule: $u x_{1}+(1-u) x_{2}=x_{0}$. In the case of the symmetric form of the free energy functional where two phases with $x_{1}=-x_{2}$ are realized, we get $h=0$ [37]. Hence, if the field $h$ becomes trivial, then the transition point can be defined.

Using the above assumptions, let us move to the stochastic case, following prescription [37]. For the one-point probability density,

$$
P_{i}(t)=\int \prod_{m \neq i} \mathcal{P}([x], t) d x_{m},
$$

the standard definition of the nearest-neighbours average

$$
\sum_{j \in n n(i)} \int \prod_{m \neq i} \mathcal{\rho}([x], t) x_{j} d x_{m}=2 d\langle x\rangle P_{i}(t)
$$

can be applied. It allows to rewrite Eq. (125) in a more useful form

$$
\frac{\partial}{\partial t} P_{i}(t)=-\frac{\partial}{\partial x_{i}} \sum_{j} \Delta_{i j}\left\langle\tilde{M}_{j}\right\rangle P_{i}(t)
$$

where

$$
\tilde{M}_{j}=M \frac{\partial F}{\partial x_{j}}-\frac{\sigma^{2}}{2} \frac{\partial M}{\partial x_{j}}+\sigma^{2} \frac{\partial}{\partial x_{j}} M
$$

With no flux condition, the average $\left\langle\tilde{M}_{j}\right\rangle$ satisfies the equation

$$
\sum_{j} \Delta_{i j}\left\langle\tilde{M}_{j}\right\rangle P_{s}\left(x_{i}\right)=0 .
$$

Taking $i=j$, dropping subscripts and using results of the deterministic analysis with $\langle\tilde{M}\rangle=h$, we obtain the mean-field stationary equation

$$
h P_{s}(x)=\left(M(x)\left[\frac{\partial V}{\partial x}-2 d D(\langle x\rangle-x)\right]-\frac{\sigma^{2}}{2} \frac{\partial M}{\partial x}+\sigma^{2} \frac{\partial}{\partial x} M\right) P_{s}(x),
$$


where we have used mean-field approximation of the Laplacian

$$
\sum_{j} \Delta_{i j} x_{j} \equiv\left(\sum_{n n(i)} x_{n n(i)}-2 d x_{i}\right) \rightarrow 2 d(\langle x\rangle-x),
$$

the mean-field value $\langle x\rangle$ should be defined self-consistently. A solution of Eq. (112) takes the form

$$
\begin{aligned}
P_{s}(x,\langle x\rangle, h) & =N \exp \left(-\frac{1}{\sigma^{2}}\left[f(x)+\frac{D^{\prime}}{2}(\langle x\rangle-x)^{2}+\right.\right. \\
+ & \left.\left.\frac{\sigma^{2}}{2} \ln M(x)-h \int \frac{d x^{\prime}}{M\left(x^{\prime}\right)}\right]\right) .
\end{aligned}
$$

where $D^{\prime}=2 d D$, next we drop the prime.

In order to determine the unknown quantities $h$ and $\langle x\rangle$, we recall that considered mean field approach is local and expresses $P_{s}$ of a field at a given site of the lattice as a function of the field $h$ and of the mean field $\langle x\rangle$ in a neighbourhood of the given cell.

In the homogeneous case (below the threshold), the mean field is the same everywhere and equals the initial value, i.e. $\langle x\rangle=x_{0}$. Hence, at the fixed mean-field value, solving the self-consistency equation,

$$
\langle x\rangle=\int x P_{s}(x,\langle x\rangle, h) d x,
$$

we obtain the constant effective field $h$. Above the threshold, the system is separated into two phases with equality $\left\langle x_{1}\right\rangle=-\left\langle x_{2}\right\rangle$, and $h$ must be the same for these two phases and must be zero. Hence, above the threshold only $\langle x\rangle$ should be defined by solving the selfconsistency equation with $P_{s}(x,\langle x\rangle, 0)$.

The values of the constant effective field $h$ are obtained as solutions of the self-consistency equation with initial concentration $x_{0}=0.2$ and shown in Fig. 17. As seen from Fig. 17, $a$, the field $h$ decreases monotonically with an increase in the control parameter $\varepsilon$. If $h$ becomes trivial, we get the transition point $\varepsilon_{T}$. After this point, $h=0$, and the mean-field value $\langle x\rangle$ can be calculated selfconsistently. It is clearly seen that the internal multiplicative noise shifts the transition point toward negative values of the control parameter $\varepsilon$. With an increase in $D$, the same circumstance is observed. The last effect is well defined: an increase in the correlation scale $r_{c}$ or spatial coupling intensity $D$ induces the ordering behaviour in the system. The former is the combined effect of the nonlinearity of the system, multiplicative character of the noise and the spatial coupling.

The noise induced effects are well seen in dependence $h\left(\sigma^{2}\right)$, shown in Fig. 17, $b$. Here, at positive values of the control parameter $\varepsilon$ (solid 

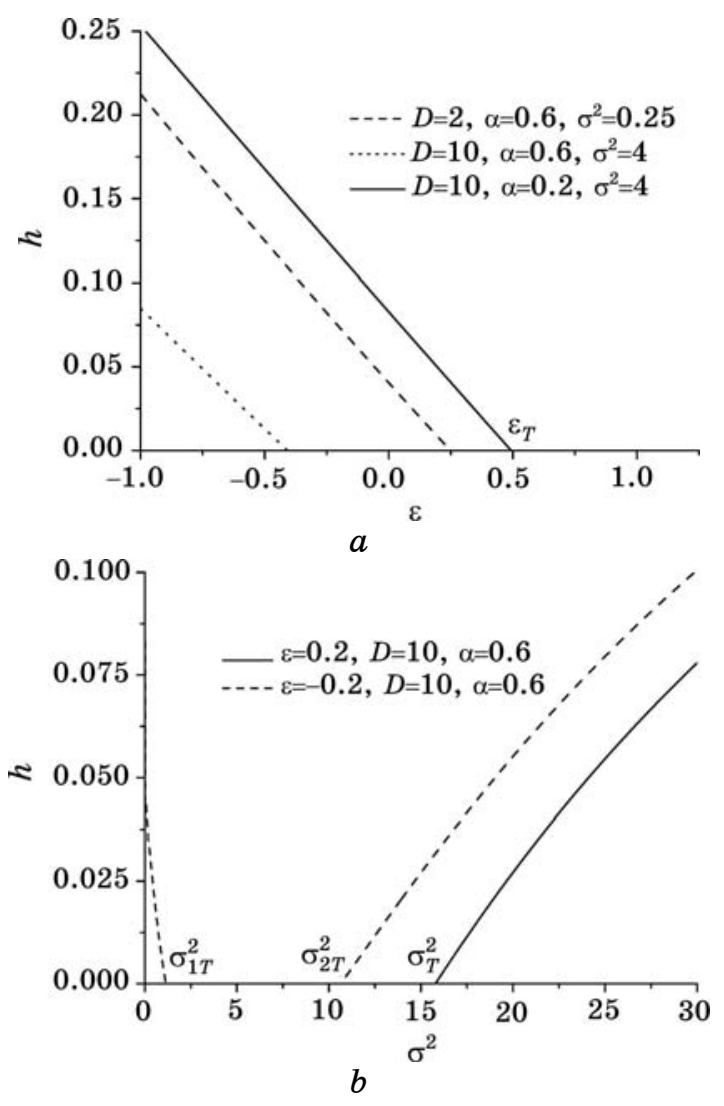

Fig. 17. Constant effective field $h$ versus control parameter $\varepsilon(a)$ and noise intensity $(b)$ at fixed initial value $x_{0}=0.2$. Other parameters are shown in legends.

line), the effective field $h$ increases from the zero value at $\sigma^{2}>\sigma_{T}^{2}$. Here one has a stable homogeneous phase $\langle x\rangle \neq x_{0}$. In the case of negative values of the control parameter (dashed line), the constant effective field $h$ becomes zero inside the domain of the noise intensities $\sigma^{2} \in\left[\sigma_{1 T}^{2}, \sigma_{2 T}^{2}\right]$. The value $h$ decreases till the first threshold $\sigma_{1 T}^{2}$, above the second one $\sigma_{2 T}^{2}$ it increases monotonically. From the formal viewpoint, the corresponding mean field value $\langle x\rangle$ obtained as a solution of the self-consistency equation (115) should be nontrivial inside the domain $\sigma^{2} \in\left[\sigma_{1 T}^{2}, \sigma_{2 T}^{2}\right]$ where the phase separation with $\left\langle x_{1}\right\rangle=-\left\langle x_{2}\right\rangle$ occurs.

Let us discuss the mean field $\langle x\rangle$ behaviour. Here, we solve the selfconsistency equation, setting $h=0$. As Figure 18, $a$, shows the mean field value changes, its value critically from zero if the parameter $\varepsilon$ increases. The critical point $\varepsilon_{c}$ is defined as a bifurcation point when 
nontrivial values $\left\langle x_{1}\right\rangle=-\left\langle x_{2}\right\rangle$ appear. The transition point $\varepsilon_{T}$ corresponds to the case when $\langle x\rangle=x_{0}$. With an increase in both the noise intensity $\sigma^{2}$ and the parameter $\alpha$, the critical point $\varepsilon_{c}$ is shifted toward negative values. The dependence of the mean field value versus noise intensity is shown in Fig. 18, $b$. Here, one can see re-entrant phase transitions at negative values of the control parameter at large spatial coupling intensity. With an increase in $\varepsilon$, the first threshold $\sigma_{1 c}^{2}$ is shifted toward small values whereas the second one $\sigma_{2 c}^{2}$ becomes larger. Transition points $\sigma_{1 T}^{2}$ and $\sigma_{2 T}^{2}$ are related to the condition $\langle x\rangle=x_{0}$. With an increase in the noise intensity at $\varepsilon>0$, the disordering phase transition is observed.

The above calculations of the effective field $h$ and the mean field value $\langle x\rangle$ allow us to obtain the corresponding phase diagrams. If the initial condition $x_{0} \neq 0$ is fixed, then one can obtain the transition lines (dash-dotted lines) which correspond to values of the control pa-
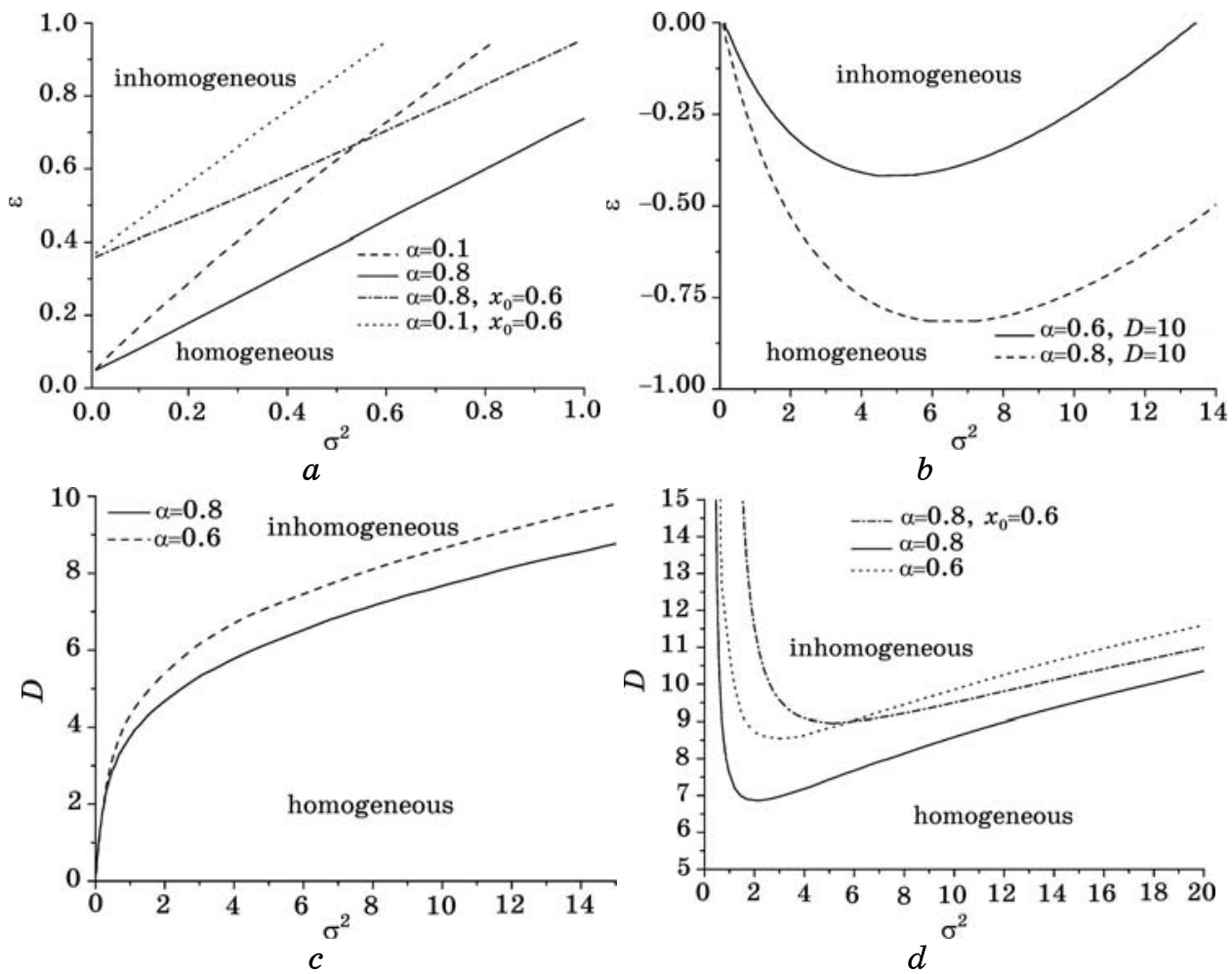

Fig. 18. Mean field value obtained as solution of Eq. (115) at $h=0:(a)$ dependence $\langle x\rangle$ versus $\varepsilon$ at different values of the spatial coupling intensity, noise intensity and the parameter $\alpha ;(b)$ dependence $\langle x\rangle$ versus $\sigma^{2}$ at $D=10$ and $\alpha=0.8$ and different values of the control parameter $\varepsilon$. 
rameter $\varepsilon_{T}$, the noise intensity $\sigma_{T}^{2}$ and the spatial coupling $D_{T}$ shown in Figs. 17, 18. The lines of critical points (solid and dashed lines) are obtained under condition when the bifurcations of the mean field $\langle x\rangle$ occur at $h=0$ (see notes in Fig. 18).

As seen from Fig. 19, $a$, an increase in the parameter $\alpha$, yielding the concentration dependent mobility, leads to a decrease in the values $\varepsilon_{c}>0$ at small spatial coupling intensity. If $\alpha$ increases, then the threshold for the noise intensity grows. It results that the bulk states with $\left\langle x_{1}\right\rangle=-\left\langle x_{2}\right\rangle$ exist at large noise intensities only if the mobility $M(x)$ decreases more abruptly. An interesting situation can be seen from Fig. 19, $b$, where the intensity of the spatial coupling is large. Here, at negative values of the control parameter $\varepsilon$, the mean field value should behave in a re-entrant manner with variation in the noise intensity. Indeed, at small and large $\sigma^{2}$ the e system is in a homogeneous state. Inside the bounded domain of the noise intensity $\sigma^{2}$, the sys-
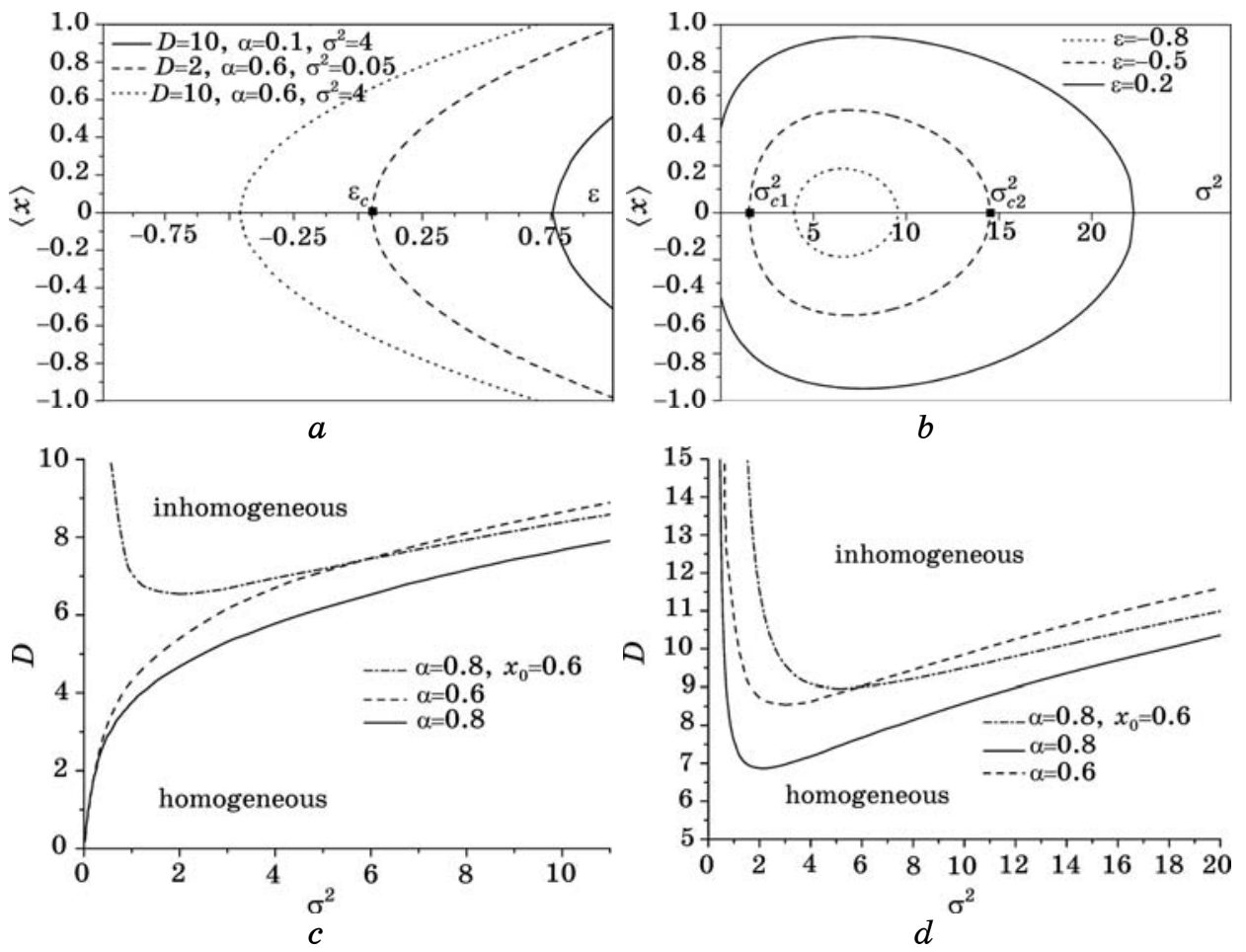

Fig. 19. Mean field diagrams at different values of the parameter $\alpha$ (solid and dashed lines of critical points plotted at $x_{0}=0, h=0$ ) and initial conditions $x_{0}$ (transition dash-dotted lines are plotted at $x_{0}=0.6, h=0$ ). Figures $(a)$ and $(b)$ correspond to $D=2.0$ and $D=10.0$, respectively. Figures $(c)$ and $(d)$ correspond to $\varepsilon=0.2$ and $\varepsilon=-0.2$, respectively. 
tem is in inhomogeneous state with $h=0$ and $\langle x\rangle \neq 0$. An increase in the parameter $\alpha$ decreases values $\varepsilon$ at which the ordered state with $\langle x\rangle \neq 0$ is observed and extends the corresponding domain of the noise intensities. Let us consider the diagram in the plane $\left(\sigma^{2}, D\right)$ shown in Fig. 19, c. Here, at positive values of the control parameter $\varepsilon$, an increase in the noise intensity destroys the state $\langle x\rangle \neq 0$, as usual. At negative $\varepsilon$, we get the re-entrant behaviour of the mean field $\langle x\rangle$, where with $\alpha$ growth transition values for the spatial coupling intensity decrease, and the domain of the noise intensity with the re-entrant behaviour extends.

Let us compare our results with computer simulations. To this end, we have computed averaged value $\left\langle M_{2}\right\rangle$, the moment $\left\langle M_{2}^{2}\right\rangle$, and the value

$$
\chi=\frac{\left\langle M_{2}^{2}\right\rangle-\left\langle M_{2}\right\rangle^{2}}{\sigma^{2}}
$$

that can be understood as a generalized susceptibility or variance. Averaging was done over 7 experiments in a stationary limit $(t \rightarrow \infty)$ at the time interval of $t=10^{4}$ to $t=2.3 \cdot 10^{4}$. Obtained results are shown in Fig. 20. From Figure 20, $a$, one can see a nonmonotonic behaviour of the order parameter $\left\langle M_{2}\right\rangle$ versus noise intensity. The variance $\chi$ in Fig. 20, $b$, shows two peaks on observable values of the noise intensity $\sigma^{2}$ in the corresponding interval. Peaks in $\chi\left(\sigma^{2}\right)$ dependence are related to two thresholds of the re-entrant phase transition. Finally, to show the entropy-driven phase transitions mechanism in the system under consideration, let us study a topology change of the distribution $P_{\mathrm{st}}(x ;\langle x\rangle, h=0)$ at fixed mean-field values $\langle x\rangle>0$. In the ordered (inhomogeneous) state, the number of extrema of the above distribution is changed. Indeed, in the domain of point $A$ (see Fig. 21), the stochastic distribution has one peak shifted toward positive values of $x$ due to
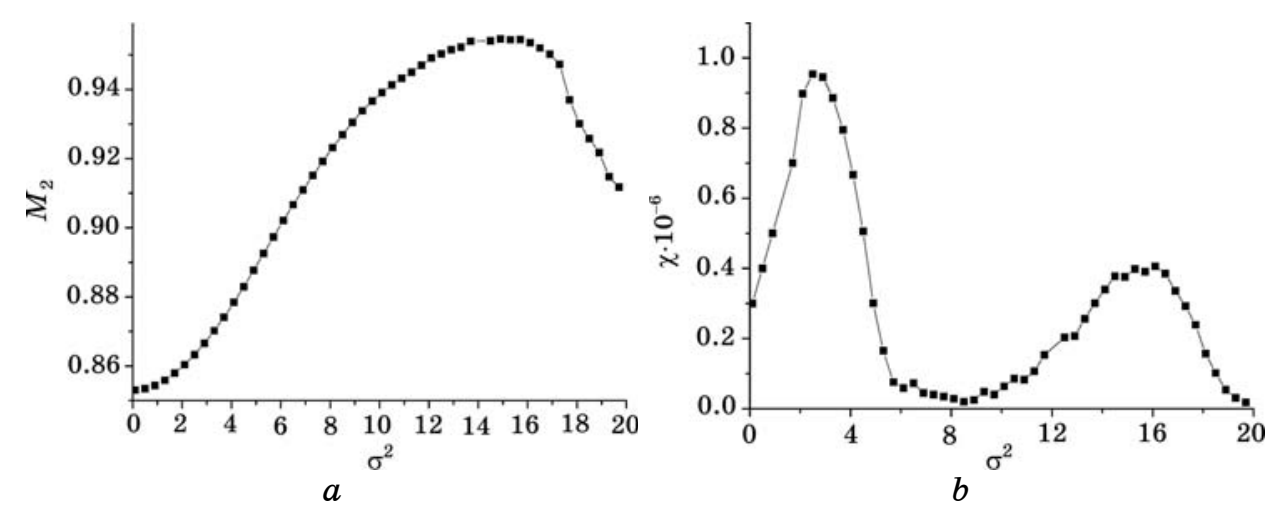

Fig. 20. Order parameter $M_{2}(a)$ and generalized susceptibility $\chi(b)$ versus noise intensity $\sigma^{2}$ at $\varepsilon=-0.5, D=10, \alpha=0.8$. 


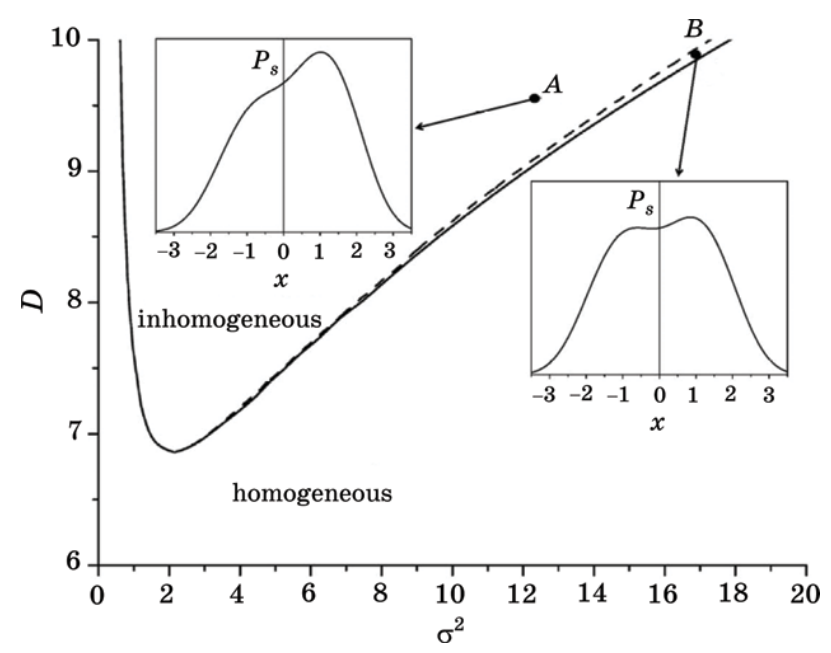

Fig. 21. Phase diagram of phase transitions showing the change of the stochastic distribution $P_{s t}(x ;\langle x\rangle>0, h=0)$ extrema. Insertions display forms of distributions of stochastic field $x$. Other parameters are as follows: $\varepsilon=-0.2, \alpha=0.8$; the mean-field value is calculated according to the values of $D$ and $\sigma^{2}$ for points $A$ and $B$, respectively.

the fact that $\langle x\rangle>0$. With an increase in the noise intensity $\sigma^{2}$, moving through the dashed line toward the point $B$, an additional peak in the distribution function appears. The dashed line corresponds to the system parameters when a double degenerated point of the stochastic distribution appears. Therefore, here we get the generalization of noise-induced transitions for extended systems.

\subsubsection{Influence of External and Internal Noise Sources}

Now, let us assume the presence of the nonequilibrium medium, which sets external fluctuations. Since the influence intensity of the medium is determined by the control parameter $\varepsilon$, we may consider an assumption about its fluctuations to be suitable for the description of real situations: $\varepsilon \rightarrow \varepsilon_{0}+\zeta(\mathbf{r}, t)$. We endow the Langevin source $\zeta(\mathbf{r}, t)$ by the Gauss properties

$$
\langle\zeta(\mathbf{r}, t)\rangle=0, \quad\left\langle\zeta(\mathbf{r}, t) \zeta\left(\mathbf{r}^{\prime}, t^{\prime}\right)\right\rangle=\tilde{\sigma}^{2} C\left(\mathbf{r}-\mathbf{r}^{\prime}\right) \delta\left(t-t^{\prime}\right)
$$

with the spatial correlation function

$$
C\left(\mathbf{r}-\mathbf{r}^{\prime}\right)=(\lambda \sqrt{2 \pi})^{-d} \exp \left(-\frac{\left|\mathbf{r}-\mathbf{r}^{\prime}\right|^{2}}{2 \lambda^{2}}\right) .
$$


Here, $\lambda$ is the correlation length of the external noise $\zeta$; $\tilde{\sigma}^{2}$ is the intensity.

As a result, we arrive at the Langevin equation in the form

$$
\frac{\partial x}{\partial t}=\nabla \cdot\left(M(x) \nabla\left[\frac{\delta \mathscr{F}[x]}{\delta x}+x \zeta(\mathbf{r}, t)\right]\right)+\nabla g(x) \xi(\mathbf{r}, t),
$$

where noises $\xi$ and $\zeta$ are assumed to be independent.

In what follows, we again pass to a discrete space, representing the continual equation (119) in the form

$$
\frac{d x_{i}}{d t}=\left(\nabla_{L}\right)_{i j} M_{j}\left(\nabla_{R}\right)_{j l}\left[\frac{\partial F}{\partial x_{l}}+x_{l} \zeta_{l}(t)\right]+\left(\nabla_{L}\right)_{i j} g_{j} \xi_{j}(t) .
$$

Here, left- and right-differences, $\left(\nabla_{L}\right)_{i j}$ and $\left(\nabla_{R}\right)_{i j}$, respectively, are consistent with definitions (90). Equation (120) can be rewritten in a more convenient form for a further analysis

$$
\frac{d x_{i}}{d t}=\left(\nabla_{L}\right)_{i j} M_{j}\left(\nabla_{R}\right)_{j l}\left[\frac{\partial F}{\partial x_{l}}\right]+\left(\nabla_{L}\right)_{i j} g_{j} \xi_{j}(t)+(\Delta)_{i j} \tilde{g}_{j} \zeta_{j}(t),
$$

where $\tilde{g}_{j}=M_{j} x_{j}$; the external noise $\zeta_{j}(t)$ obeys Gaussian properties: $\left\langle\zeta_{i}(t)\right\rangle=0,\left\langle\zeta_{i}^{j}(t) \zeta_{j}\left(t^{\prime}\right)\right\rangle=2 \tilde{\sigma}^{2} C_{|i-j|} \delta\left(t-t^{\prime}\right)$, where $C_{|i-j|}$ is a discrete representation of the spatial correlation function $C\left(\left|\mathbf{r}-\mathbf{r}^{\prime}\right|\right)$.

Early Stage of the System Evolution with Two Noises. As done in previous sections, we will study the instability of the state $x(\mathbf{r}, t)=0$, taking only into account the linear terms in Eq. (120). In this case, the dynamical equation for the structure function takes the form

$$
\begin{gathered}
\frac{d S(k, t)}{d t}=-\omega(k) S(k, t)+2 \sigma^{2} k^{2}- \\
-\frac{2 \alpha \sigma^{2} k^{2}}{(2 \pi)^{d}} \int d \mathbf{q} S(q, t)+\frac{2 k^{2} \tilde{\sigma}^{2}}{(2 \pi)^{d}} \int d \mathbf{q} G(q) S(q, t),
\end{gathered}
$$

where $G(q)$ is the Fourier transform of the external-noise correlation function $C\left(\mathbf{r}-\mathbf{r}^{\prime}\right)$. The dispersion relation reads [84]

$$
\omega(k)=k^{2}\left(\left[D-\tilde{\sigma}^{2} C_{1}\right] k^{2}-\varepsilon+\sigma^{2}-\tilde{\sigma}^{2} 2 d\left(C_{0}-C_{1}\right)\right),
$$

where $\tilde{\sigma}$ is the intensity as in Eq. (117). The dispersion relation indicates that for $\omega(k)>0$, the homogeneous null state is stable. This occurs for $-\varepsilon+\alpha \sigma^{2}+\tilde{\sigma}^{2}\left[\nabla^{2} C(|\mathbf{r}|)\right]_{r=0}>0$, so we can define an effective control parameter

$$
\varepsilon_{\mathrm{ef}}=-\varepsilon+\alpha \sigma^{2}-\tilde{\sigma}^{2}\left[\nabla^{2} C(|\mathbf{r}|)\right]_{r=0}>0,
$$


such that the homogeneous null state is stable for $\varepsilon_{\text {ef }}>0$. Therefore, the onset of stability is now given by $\varepsilon_{t}=\alpha \sigma^{2}-\tilde{\sigma}^{2}\left[\nabla^{2} C(|\mathbf{r}|)\right]_{r=0}$, which in a discrete space is $\varepsilon_{t}=\alpha \sigma^{2}+\tilde{\sigma}^{2} 2 d\left(C_{0}-C_{1}\right)$. Moreover, the expression for $\omega(k)$ has a noise dependent term that can be considered as a modification of the spatial coupling parameter. Thus, we can define an effective spatial coupling parameter $D_{\text {ef }}=D-\tilde{\sigma}^{2} C_{1}[90]$.

Mean Field Analysis of the Stationary Case. Let us consider the stationary case. To this end, we can use the standard procedure described above to find the stationary distribution as a solution of the corresponding Fokker-Planck equation. The total probability density functional obeys the equation [37, $61,62,67]$

$$
\begin{gathered}
\frac{\partial \mathcal{P}}{\partial t}=\sum_{i j} \frac{\partial}{\partial x_{i}} \Delta_{i j}\left(M_{j}\left[-\frac{\partial V}{\partial x_{j}}+D \sum_{r} \Delta_{j r} x_{r}\right]-\right. \\
\left.-\sigma^{2} g_{j} \frac{\partial}{\partial x_{j}} g_{j}+\tilde{\sigma}^{2} \tilde{g}_{j} \sum_{m, n} \frac{\partial}{\partial x_{n}} \Delta_{m n} C_{|j-n|} \tilde{g}_{n}\right) \mathcal{P} .
\end{gathered}
$$

To perform mean-field calculations, we exploit the one-point probability density satisfying the equation

$$
\frac{\partial P_{i}(t)}{\partial t}=\frac{\partial}{\partial x_{i}} \sum_{j} \Delta_{i j}\left\langle\tilde{M}_{j}\right\rangle P_{i}(t)
$$

where

$$
\tilde{M}_{j}=M_{j}\left[-\frac{\partial V}{\partial x_{j}}+D \sum_{r} \Delta_{j r} x_{r}\right]-\sigma^{2} g_{j} \frac{\partial}{\partial x_{j}} g_{j}+\tilde{\sigma}^{2} \tilde{g}_{j} \sum_{m, n} \frac{\partial}{\partial x_{n}} \Delta_{m n} C_{|j-n|} \tilde{g}_{n} .
$$

Assuming that a stationary distribution $P_{s}\left(x_{i}\right)$ can be obtained under no flux conditions, the quantity $\left\langle\tilde{M}_{j}\right\rangle$ should obey the equation

$$
\sum_{j} \Delta_{i j}\left\langle\tilde{M}_{j}\right\rangle P_{s}\left(x_{i}\right)=0 .
$$

Following the standard procedure, one can find that the mean-field distribution function can be computed directly from the stationary equation

$$
\begin{gathered}
-h P_{s}(x)=\left(M(x)\left[-\frac{\partial V}{\partial x}+2 d D(\langle x\rangle-x)\right]-\sigma^{2} g(x) \frac{\partial}{\partial x} g(x)+\right. \\
\left.+2 d \tilde{\sigma}^{2} \tilde{g}(x)\left[C_{1} \tilde{g}(\langle x\rangle) \frac{\partial}{\partial x}-C_{0} \frac{\partial}{\partial x} \tilde{g}(x)\right]\right) P_{s}(x),
\end{gathered}
$$

where, according to the mean-field approximation, one can put $\langle g(x)\rangle ; g(\langle x\rangle)$ [37], and drop the prime for $D^{\prime}=2 d D$. Therefore, for the 
stationary distribution one has

$$
P_{s}(x,\langle x\rangle, h)=N \exp \left(\int d x^{\prime} \frac{\Omega\left(x^{\prime} ;\langle x\rangle ; h\right)}{\Theta\left(x^{\prime} ;\langle x\rangle\right)}\right),
$$

where

$$
\Omega(x ;\langle x\rangle ; h)=M(x)\left[-\frac{\partial f(x)}{\partial x}+D(\langle x\rangle-x)\right]-\frac{\sigma^{2}}{2} \frac{\partial M(x)}{\partial x}-d \tilde{\sigma}^{2} C_{0} \frac{\partial \tilde{g}^{2}(x)}{\partial x}+h,
$$
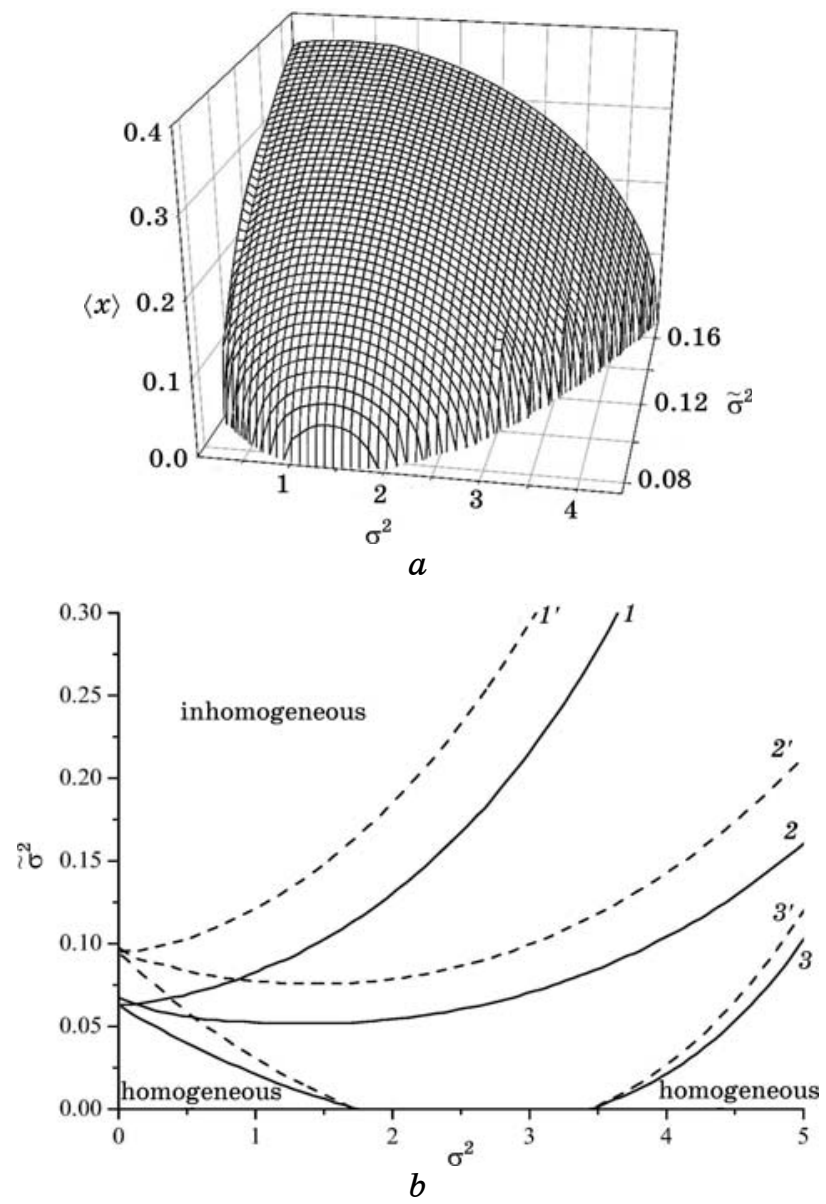

Fig. 22. Dependence of the mean field versus the noise intensities (internal and external) at $\alpha=0.4, \varepsilon=-0.2, D=10(a)$ and the phase diagram at different values of the correlation radius $\lambda$, spatial coupling parameter $D$ and $\alpha(b)$ (curves $1-3$ correspond to $\lambda=0$, whereas curves $1^{\prime}-3^{\prime}$ relate to $\lambda=1: 1$ and $1^{\prime}-$ $D=8.3, \alpha=0.4 ; 2$ and $2^{\prime}-D=10, \alpha=0.4,3$ and $3^{\prime}-D=8.3, \alpha=0.6$ ). 


$$
\Theta(x ;\langle x\rangle)=\sigma^{2} M(x)+2 d \tilde{\sigma}^{2} \tilde{g}(x)\left(C_{0} \tilde{g}(x)-C_{1} \tilde{g}(\langle x\rangle)\right) .
$$

Unknown field $h$ and $\langle x\rangle$ can be obtained form the self-consistency equation described above.

Let us consider an influence of spatial correlations $\lambda$ of external noise on a position of the critical points. Corresponding phase diagrams are shown in Fig. 22 at different values of $D$ and $\alpha$ (curves 1-3) and at different values of spatial correlation radius $\lambda$.

As figure shows an increase in $D$ leads to decrease in critical noise intensity magnitudes $\tilde{\sigma}^{2}$ and promotes re-entrance in phase transition picture (see curves 1,2). The same situation is observed when the parameter $\alpha$ increases (see curves 1,3). An increase in the correlation radius of the external noise results in increase in its critical values (see curves $\left.1^{\prime}-3^{\prime}\right)$. It leads to the fact that area of re-entrant behaviour of the order parameter shrinks.

Strong Coupling Limit. Considering a strong coupling limit, we assume $D \rightarrow \infty$ and neglect all possible correlations, i.e. $\langle\varphi(x)\rangle ; \varphi(\langle x\rangle)$. Hence, the stationary distribution functions are given by the mean field approach for each phase and have the form $P_{s}(x,\langle x\rangle)=\delta(x-\langle x\rangle)$. Next, to obtain an equation for the effective field $h$, we integrate Eq. (112) and find

$$
h=M(\langle x\rangle) V^{\prime}(\langle x\rangle)-\left(\sigma^{2} / 2\right) M^{\prime}(\langle x\rangle),
$$

where prime denotes derivative with respect to the argument.

In the homogeneous case, $\varepsilon<\varepsilon_{T}$, we have $\langle x\rangle=x_{0}$, and $h$ becomes a function of the initial conditions. If the value $x_{0}$ is fixed, then the field $h$ decreases with an increase in $\varepsilon$ until it reaches the null value, and increases from the null value with an increase in $\sigma^{2}$. As it follows from the Eq. (132) and mean field analysis, no re-entrance can be found in strong coupling limit. Therefore, a re-entrant behaviour of the mean-field value is realized only at finite magnitudes of the spatial coupling intensity $D$.

In the case of $\varepsilon>\varepsilon_{T}$, we have $h=0$. Solutions of Eq. (132) give values for the bulk phases

$$
\langle x\rangle_{1,2}= \pm \frac{\sqrt{2 \alpha}}{2 \alpha}\left(-1-\alpha \varepsilon+\sqrt{(1-\alpha \varepsilon)^{2}+4 \alpha^{2} \sigma^{2}}\right)^{1 / 2} .
$$

The corresponding transition lines are defined by condition $\langle x\rangle_{1}=x_{0}$ that leads to

$$
\varepsilon_{T}=\frac{\alpha^{2} \sigma^{2}-x_{0}^{2}\left(1+\alpha x_{0}^{2}\right)}{1+\alpha x_{0}^{2}} .
$$

The critical point (for $x_{0}=0$ ) is 


$$
\varepsilon_{c}=\alpha^{2} \sigma^{2},
$$

which coincides with linear stability analysis. (One should not be confused comparing our results with results in Refs [76, 84]. We consider the internal multiplicative noise influence, whereas in Refs [76, 84] systems with an external multiplicative noise and conserved dynamics were studied. In above papers, it was noted that the critical value $\varepsilon_{c}$ differs from the obtained in Eq. (135) by the multiplier $2 d$. Such multiplier arises only if an external noise is introduced. If we follow the procedure described in Ref. [76] and consider external fluctuations, supposing $\varepsilon \rightarrow \varepsilon_{0}+\sigma \eta(\mathbf{r}, t)$, where $\eta(\mathbf{r}, t)$ is the Gaussian noise, then we will recover results with the multiplier $2 d$.) It follows that the strong coupling limit sets the critical values for both the control parameter and the noise intensity, which correspond to $k_{c}=0$. In other words, at $\varepsilon>\alpha \sigma^{2}$, unstable modes start to growth. Moreover, we can define values $\alpha<\alpha_{c}=\varepsilon / \sigma^{2}$ at which phase separation exhibits spatial patterns. Due to $\varepsilon \in[-1,1]$ and $\sigma^{2}>0, \alpha \geq 0$, one gets that $\alpha_{c}$ decreases with an increase in the noise intensity $\sigma^{2}$. At negative values of the control parameter, we get the phase separation with no patterning.

For the system with two stochastic sources, one can find that critical value for the control parameter is renormalized as

$$
\varepsilon_{c}=\alpha \sigma^{2}-2 d \tilde{\sigma}^{2}\left(C_{0}-C_{1}\right) .
$$

From this, it follows that two stochastic sources compete with each other. Here, we get shift of the critical point with the multiplier $2 d$ related to the noise intensity $\tilde{\sigma}^{2} C_{0}$ and spatial correlations $C_{1}$.

\subsection{Stochasticity in Hyperbolic Transport}

In this Section, we focus on a more conceptual question related to the fluctuations of the flux $\mathbf{J}$ or, more concretely, to their conceptual interpretation. We shall see that, depending on the value of the relaxation time $\tau_{D}$ and of the observational time scale, such fluctuations can be interpreted in two different ways described in Ref. [40]. To discuss these ideas, we must recall some results concerning hydrodynamic stochastic noise.

Hyperbolic Transport with Noise. We discuss now the stochastic noise in a system,

$$
\tau \ddot{\alpha}+\dot{\alpha}=-L \alpha+\zeta,
$$

which may generally represent the hyperbolic transport (3) with noise. Taking into account both independent variables $\alpha$ and $\dot{\alpha} \equiv \beta$, Eq. (137) may be written as 


$$
\dot{\alpha}=\beta+\tilde{\zeta}_{\alpha}, \quad \dot{\beta}=-\frac{L}{\tau} \alpha-\frac{1}{\tau} \beta+\tilde{\zeta}_{\beta} .
$$

The set of equations (138) represents the second-order equation (137) as two first-order evolution equations, in a way that the system becomes Markovian. In Eq. (138), $\zeta_{\alpha}$ and $\zeta_{\beta}$ are the respective stochastic sources, whose second-order moments have to be obtained. The correlator of fluctuating terms is defined as follows

$$
\left\langle\tilde{\zeta}(t) \tilde{\zeta}\left(t^{\prime}\right)\right\rangle=\left[\begin{array}{cc}
0 & -1 \\
\frac{L}{\tau} & \frac{1}{\tau}
\end{array}\right]\left[\begin{array}{cc}
\left\langle\alpha^{2}\right\rangle_{\text {eq }} & \langle\alpha \beta\rangle_{\text {eq }} \\
\langle\beta \alpha\rangle_{\text {eq }} & \left\langle\beta^{2}\right\rangle_{\text {eq }}
\end{array}\right]+\left[\begin{array}{cc}
\left\langle\alpha^{2}\right\rangle_{\text {eq }} & \langle\alpha \beta\rangle_{\text {eq }} \\
\langle\beta \alpha\rangle_{\text {eq }} & \left\langle\beta^{2}\right\rangle_{\text {eq }}
\end{array}\right]\left[\begin{array}{cc}
0 & \frac{L}{\tau} \\
-1 & \frac{1}{\tau}
\end{array}\right] .
$$

In Equation (139), we have $\langle\alpha \beta\rangle_{\text {eq }}=\langle\beta \alpha\rangle_{\text {eq }}=0$ because $\alpha$ and $\dot{\alpha}$ have opposite time-reversal symmetry. Thus, it is found

$$
\left\langle\tilde{\zeta}_{\alpha}^{2}\right\rangle=0, \quad\left\langle\tilde{\zeta}_{\beta}^{2}\right\rangle=\frac{2}{\tau}\left\langle\beta^{2}\right\rangle_{\text {eq }}, \quad\left\langle\tilde{\zeta}_{\alpha} \tilde{\zeta}_{\beta}\right\rangle=\left\langle\tilde{\zeta}_{\beta} \tilde{\zeta}_{\alpha}\right\rangle=\frac{L}{\tau}\left\langle\alpha^{2}\right\rangle_{\text {eq }}-\left\langle\beta^{2}\right\rangle_{\text {eq }} \cdot
$$

To obtain the second moment of equilibrium fluctuations of $\alpha$ and $\beta$, we assume, as in Section 2, that $\alpha$ and $\beta$ are independent variables. Then, including both of these variables into the entropy, one can write

$$
S(\alpha, \beta)=S_{\text {eq }}-\frac{1}{2} A \alpha^{2}-\frac{1}{2} B \beta^{2},
$$

where only second-order terms have been considered. According to Eq. (7), the probability of fluctuations is described by

$$
\operatorname{Pr}(\alpha, \beta) \sim \exp \left[-\frac{A}{2 k_{B}} \alpha^{2}-\frac{B}{2 k_{B}} \beta^{2}\right] .
$$

Note that, in Eq. (142), we have identified the fluctuations $\delta \alpha$ and $\delta \beta$ with $\alpha$ and $\beta$, respectively, because their equilibrium average values are zero for both of them due to the form of the evolution equations (138). Then, in equilibrium, we have

$$
\left\langle\alpha^{2}\right\rangle_{\text {eq }}=\frac{k_{B}}{A}, \quad\left\langle\beta^{2}\right\rangle_{\text {eq }}=\frac{k_{B}}{B} .
$$

To evaluate the ratio $A / B$, we obtain the entropy production corresponding to Eq. (141). This one yields

$$
d S / d t=-A \alpha \dot{\alpha}-B \beta \dot{\beta}=-A \alpha \dot{\alpha}-B \dot{\alpha} \ddot{\alpha}=-\dot{\alpha}[A \alpha+B \ddot{\alpha}] \geq 0 .
$$

From this, and by following the usual methods of nonequilibrium 
thermodynamics [91], it follows the linear relation between thermodynamic flux $-\dot{\alpha}$ and its conjugated force $A \alpha+B \ddot{\alpha}$. This is

$$
A \alpha+B \ddot{\alpha}=-\mu \dot{\alpha} .
$$

Comparison of Eq. (145) with Eq. (137) yields $B=\tau, \mu=1$, and $A=L$. Thus, as follows, the second moments of $\alpha$ and $\beta$ are related by

$$
\frac{\left\langle\alpha^{2}\right\rangle_{\mathrm{eq}}}{\left\langle\beta^{2}\right\rangle_{\mathrm{eq}}}=\frac{B}{A}=\frac{\tau}{L} .
$$

Introducing this relation into Eq. (140) it is found

$$
\left\langle\tilde{\zeta}_{\alpha}^{2}\right\rangle=0, \quad\left\langle\tilde{\zeta}_{\alpha} \tilde{\zeta}_{\beta}\right\rangle=\left\langle\tilde{\zeta}_{\alpha} \tilde{\zeta}_{\beta}\right\rangle=0, \quad\left\langle\tilde{\zeta}_{\beta}^{2}\right\rangle=\frac{2}{\tau}\left\langle\beta^{2}\right\rangle_{\text {eq }}=\frac{2 L}{\tau^{2}}\left\langle\alpha^{2}\right\rangle_{\text {eq }} .
$$

Since $\left\langle\tilde{\zeta}_{\alpha}^{2}\right\rangle=0$, the first equation in Eq. (138) may be introduced into the second one. Therefore, we get

$$
\tau \ddot{\alpha}+\dot{\alpha}=-L \alpha+\tau \tilde{\zeta}_{\beta},
$$

with

$$
\left\langle\zeta_{\beta}(0) \zeta_{\beta}(t)\right\rangle=\left\langle\tau \tilde{\zeta}_{\beta}(0) \tau \tilde{\zeta}_{\beta}(t)\right\rangle=2 L\left\langle\alpha^{2}\right\rangle_{\mathrm{eq}} \cdot
$$

Thus, the expression for the noise keeps the same form as in the case with $\tau=0$. This is in agreement with the ideas of fluctuationdissipation, which relate the fluctuations to the dissipative part of the equation [the term in $L$ in Eq. (148)].

The transition from noisy hyperbolic transport described by equation (137) to Langevin equation $\dot{\alpha}=-L \alpha+\zeta$ might be analyzed by considering the generalized entropy (141) in the following form

$$
S(\alpha, \beta)=S_{\text {eq }}-\frac{A}{2} \alpha^{2}-\frac{A \tau}{2 L} \beta^{2} .
$$

With $\tau \rightarrow 0$, the last term in $\beta^{2}$ disappears together with the term in $\ddot{\alpha}$ in Eq. (137). In this case, the dynamics of $\alpha$ is described by a simple relaxation with a temporal constant given by $L^{-1}$. One interesting situation may be found when $\tau<<L^{-1}<<1$. In this case, $\alpha$ decays slowly and $\beta$ decays fast. Assume, for instance, that $L \approx 1 \mathrm{~s}^{-1}$ and $\tau \sim 10^{-3} \mathrm{~s}$. The typical relaxation of $\alpha$ will be of the order of 1 second and $\beta$ will decay in a millisecond scale. In this case, $\tilde{\zeta}_{\beta}$ describes the effect of all the variables whose relaxation time is much less than $10^{-3}$ $\mathrm{s}$, in such a way that they may be assumed to decay instantaneously in comparison with $\beta$.

Fluctuations or Independent Variable. Now, consider a special system 
with two independent variables $\alpha$ and $\beta$ in such a way that the relaxation rate of $\beta$ is characterized by its high but finite value. In this case, one may write $\delta \beta \equiv \beta+L \alpha$, i.e. $\beta=-L \alpha+\delta \beta$, where $\delta \beta$ being the independent part of $\beta$. This part of $\beta$ is orthogonal to the slow subspace generated by $\alpha$. Then, we may write

$$
\dot{\alpha}=-L \alpha+\delta \beta, \quad \delta \dot{\beta}=-\frac{1}{\tau} \delta \beta .
$$

and

$$
\langle\delta \beta(0) \delta \beta(t)\rangle=\frac{L k_{B}}{A \tau} e^{-|t| / \tau}=\frac{L}{\tau}\left\langle\alpha^{2}\right\rangle e^{-|t| / \tau} .
$$

The following three cases may be outlined in considering Eqs (151) and (152).

(i) If $\tau$ is sufficiently short, $\delta \beta$ acts as a 'noise' in the equation for $\alpha$ (the first equation of system (151)).

(ii) If $\tau$ is not completely negligible as compared to $L^{-1}, \delta \beta$ acts as a coloured noise.

(iii) In the limit $\tau \rightarrow 0$, one has

$$
\langle\delta \beta(0) \delta \beta(t)\rangle \rightarrow 2 L\left\langle\alpha^{2}\right\rangle_{\text {eq }} \delta(t) .
$$

Thus, it is seen that the transition of Eq. (150) and (151) from small $\tau$ to vanishing $\tau$ is conceptually interesting. It is illustrative of how the variable $\beta$ (i.e., $\dot{\alpha}$ ) goes from an independent variable with its own dynamics to a purely Markovian stochastic noise. In physical terms, the frontier between small $\tau$ and vanishing $\tau$ is settled by the time scale one is able to measure. For instance, if $L=1 \mathrm{~s}^{-1}$ and $\tau=10^{-8} \mathrm{~s}$, the system will have two independent variables, $\alpha$ and $\dot{\alpha}$, for an observer which is able to measure picoseconds $\left(10^{-12} \mathrm{~s}\right)$. However, it will have only one independent variable, $\alpha$, plus a stochastic noise, in a form of $\dot{\alpha}$, for an observer which is only able to measure milliseconds $\left(10^{-3} \mathrm{~s}\right)$. The latter observer will be able to work in the 'adiabatic' approximation with very slow $\alpha$ in comparison with $\beta$.

The situation described by Eqs (1) and (2) is interesting from this perspective. For negligible values of the diffusion relaxation time $\tau_{D}$, one should use the Landau-Lifshitz formalism for the fluctuating hydrodynamics [28] and write

$$
\frac{\partial c}{\partial t}=D \nabla^{2} c+\zeta_{c}
$$

where the stochastic noise $\zeta_{c}$ is interpreted as

$$
\zeta_{c}=-\nabla \cdot \delta \mathbf{J}
$$


with $\delta \mathbf{J}$ being a fluctuating part of the diffusion flux, i.e.

$$
\mathbf{J}=-D \nabla c+\delta \mathbf{J} .
$$

According to the Landau-Lifshitz approach [28], one has

$$
\langle\delta \mathrm{J}(0) \delta \mathrm{J}(t)\rangle=2 k_{B} D T \delta(t) .
$$

Assume, in contrast, that $\tau_{D}$ is small but still measurable. In this case, we get

$$
\frac{\partial c}{\partial t}=-\nabla \cdot \mathbf{J}, \quad \tau_{D} \frac{\partial \mathbf{J}}{\partial t}+\mathbf{J}=-D \nabla c+\zeta_{J},
$$

with $c$ and $\mathbf{J}$ being independent variables of the entropy given by the generalized Gibbs equation (8). The second moments of the fluctuation of $c$ and $\mathbf{J}$ are given by Eq. (11). The noise $\zeta_{J}$ would correspond to values relaxing in time scales much shorter than $\tau_{D}$, in such a way that it may be considered as Markovian noise:

$$
\left\langle\zeta_{J}(0) \zeta_{J}(t)\right\rangle=2 k_{B} D T \delta(t) .
$$

In the limit of vanishing $\tau_{D}$, the fast part of $\mathbf{J}$ becomes a stochastic noise, and we get $\zeta_{c}$ in Eq. (154) described by Eq. (157).

Still another form to discuss the interpretation of noise in the context of Eq. (137) is to write Eq. (138) without any added noise, i.e., in the following form

$$
\dot{\alpha}=\beta, \quad \dot{\beta}=-\frac{L}{\tau_{D}} \alpha-\frac{\beta}{\tau_{D}} .
$$

This set of equations may be integrated to give

$$
\dot{\alpha}(t)=-\int_{t_{0}}^{t} M\left(t-t^{\prime}\right) \alpha\left(t^{\prime}\right) d t^{\prime}+\beta(t),
$$

with the memory function

$$
M\left(t-t^{\prime}\right)=\frac{L}{\tau_{D}} \exp \left(-\frac{t-t^{\prime}}{\tau_{D}}\right),
$$

and the exponential relaxation

$$
\beta(t)=\dot{\alpha}\left(t_{0}\right) \exp \left(-\frac{t-t_{0}}{\tau_{D}}\right) .
$$

In this case, the 'noise' is due to the uncertainty in the value of $\dot{\alpha}(t)$ at the initial time $t_{0}$. 
Mori's expression for the fluctuation-dissipation theorem states that

$$
\left\langle\beta(t) \beta\left(t^{\prime}\right)\right\rangle=M\left(t-t^{\prime}\right)\left\langle\alpha^{2}\right\rangle_{\mathrm{eq}} .
$$

Indeed, if we use the result (146), we obtain that

$$
\left\langle\dot{\alpha}^{2}\left(t_{0}\right)\right\rangle_{\mathrm{eq}} \equiv\left\langle\beta^{2}\left(t_{0}\right)\right\rangle_{\mathrm{eq}}=\left\langle\alpha^{2}\right\rangle_{\mathrm{eq}} \frac{L}{\tau} .
$$

Combination of Eq. (165) with Eqs (162) and (163) gives Eq. (164).

Again, when $\tau$ becomes negligible, $M\left(t-t^{\prime}\right)$ given in Eq. (162) becomes

$$
M\left(t-t^{\prime}\right)=L \delta\left(t-t^{\prime}\right)
$$

and Eq. (161) becomes

$$
\dot{\alpha}(t)=-L \alpha(t)+\zeta_{\alpha},
$$

with

$$
\left\langle\zeta_{\alpha}^{2}\right\rangle=2 L\left\langle\alpha^{2}\right\rangle
$$

Note that, in the limit (166), we consider $t>t^{\prime}$, whereas in the limit (168), a factor 2 appears because one considers $\left|t-t^{\prime}\right|>0$ rather than $t-t^{\prime}>0$, i.e. one considers both $t-t^{\prime}>0$ and $t^{\prime}-t>0$.

\subsection{Stochastic Hyperbolic Model for Spinodal Decomposition}

Considering the stochastic hyperbolic model, let us start with a set of equations for the local concentration and the flux in the forms as follow:

$$
\begin{gathered}
\frac{\partial}{\partial t} x=-\nabla \cdot \mathbf{J}, \\
\tau_{D} \frac{\partial}{\partial t} \mathbf{J}=-\mathbf{J}-M \nabla \frac{\delta \mp[x]}{\delta x}+\zeta(x ; \mathbf{r}, t) .
\end{gathered}
$$

Here, $\tau_{D}$ is a relaxation time for the flux $\mathbf{J}$. In further analysis, let us introduce a dimensionless time $t^{\prime}=t / \tau_{x}$ and operator $\nabla^{\prime}=\ell_{x}^{-1} \nabla_{x}$, where scales $\ell_{x}$ and $\tau_{x}$ are introduced (usually, the time scale for such transition is defined through the Debye frequency $\omega_{D}$, diffusion energy $E_{\text {diff }}$ and temperature $T$ as $\tau_{x}=\omega_{D}^{-1} \exp \left(E_{\text {diff }} / T\right)$ ). If the diffusion is caused by the vacancy mechanism, then the quantity $E_{\text {diff }}$ is energy for migration of vacancies. Next, let us move to dimensionless quantities $J^{\prime}=J / V_{D}$ with $V_{D}=\ell_{x} / \tau_{x}, M^{\prime}=M / M_{0}, \mathscr{F}^{\prime}=\mathscr{F}^{\prime} / \mathscr{F}_{0}$, 
$\zeta^{\prime}=\zeta / V_{D}$ for flux, mobility and free energy, and noise respectively. Inserting such quantities into Eq. (169), dropping the prime, and assuming $M_{0} \mathscr{F}_{0}=V_{D} \ell_{x} \tau_{x}$, we reduce the set of two first-order time equation to the second-order equation in the form of

$$
\delta \frac{\partial^{2} x}{\partial t^{2}}+\frac{\partial x}{\partial t}=\nabla \cdot(M(x) \nabla \mu+g(x) \zeta(\mathbf{r}, t)),
$$

where $\delta=\tau_{D} / \tau_{x}$ states the ratio of relaxation times for $g(x)=\sqrt{M(x)}$. At $\delta<1$, one arrives at the parabolic model for the spinodal decomposition discussed previously in Section 4.1.

In our consideration, it can be shown that two conjugate variables, as the local concentration and the flux, should be considered as commensurable variables as a special case. Moreover, we will explain that even the flux is supposed to be fast variable our results leads to the well-known picture of nonlinear dependence of an amplification rate at early stages, whereas at late stages (stationary case), the hyperbolicity of the model does not affect on the system behaviour essentially.

\subsubsection{Early Stages Analysis}

Let us consider an early stage of the system evolution. As done before, we can obtain an evolution equation for the spherically averaged structure function $S(k, t)$. In the following, we use the special case of the white noise assumption, $C\left(\mathbf{r}-\mathbf{r}^{\prime}, t-t^{\prime}\right) \rightarrow \sigma^{2} \delta\left(\mathbf{r}-\mathbf{r}^{\prime}\right) \delta\left(t-t^{\prime}\right)$. After some algebra, one gets

$$
\begin{gathered}
\left(\delta \frac{d^{2}}{d t^{2}}+\frac{d}{d t}\right) S(k, t)=-k^{2}\left(D k^{2}-\varepsilon+\alpha \sigma^{2}\right) S(k, t)+ \\
+2 \sigma^{2} k^{2}-2 \alpha \sigma^{2} k^{2} \frac{1}{(2 \pi)^{d}} \int d \mathbf{q} S(q, t) .
\end{gathered}
$$

This equation is reduced to Eq. (63) in the deterministic case $\left(\sigma^{2}=0\right)$, whereas in the case of $\delta<<1$ and $\alpha=0$ one arrives at the Cahn-Hilliard-Cook equation $[12,59]$. It can be seen that the dispersion relation now takes the form

$$
\omega(k)=-\frac{i}{2 \delta} \pm \sqrt{\frac{k^{2}\left(k^{2} D-\varepsilon+\alpha \sigma^{2}\right)}{\delta}-\frac{1}{4 \delta^{2}}},
$$

which, in the case of the local equilibrium, $\delta<<1$, is as follows:

$$
\left.\omega(k)\right|_{\delta=1}=-i(2 \delta)^{-1}\left[1 \pm 2 \delta k^{2}\left(k^{2} D-\varepsilon+\alpha \sigma^{2}\right)\right] .
$$

At $k<k_{c}$, the imagine part of the frequency (173) 


$$
\mathfrak{I}(\omega(k))=(2 \delta)^{-1}\left[1 \pm \sqrt{1-4 \delta k^{2}\left(k^{2} D-\varepsilon+\alpha \sigma^{2}\right)}\right]
$$

can be used to obtain maximal wave-vector amplitude $k_{\mathrm{m}}$.

To obtain a normalized amplification rate, we need to compare dispersion relations obtained for both parabolic and hyperbolic stochastic models. To that end, we use the quantity $\omega_{\mathrm{CHC}}\left(k_{\mathrm{m}}\right)$ obtained from the parabolic Cahn-Hilliard-Cook model with multiplicative fluctuations as a normalization factor, $k_{\mathrm{m}}=\sqrt{\left(\varepsilon-\alpha \sigma^{2}\right) / 2 D}$ (see Subsection 4.1.1). Acting in such a manner, the normalized amplification rate takes the form $\omega_{\text {hyp }}^{*}(q) / q^{2}=\omega_{\text {hyp }}(k) / \omega_{\text {CHC }}\left(k_{\mathrm{m}}\right) / q^{2}$, where $q=k / k_{c}$, and dispersion relation $\omega_{\text {hyp }}(k)$ is taken from Eq. (175) as $\omega_{\text {hyp }}(k)=\mathfrak{I}(\omega(k))$. Therefore, for the stochastic hyperbolic model one has

$$
\omega^{*}(q) / q^{2}=\frac{2 D}{\delta q^{2}} \frac{\sqrt{1+4 \delta \frac{\left(\varepsilon-\alpha \sigma^{2}\right)^{2}}{D} q^{2}\left(1-q^{2}\right)}-1}{\left(\varepsilon-\alpha \sigma^{2}\right)^{2}} .
$$

In such a case, both parabolic and hyperbolic normalized amplification rates take values: $\omega(q) / q^{2}=0$ at $q=1$ and $\omega(q) / q^{2}=4$ at $q=0$. At $\delta \rightarrow 0$ (transition to the one slow variable (parabolic) model), one arrives at the linear dependence $\omega(q) / q^{2}$ versus $q^{2}$.

The corresponding dependence from Eq. (176) is shown in Fig. 23. Comparing different curves, one can see that in the nonlinear behaviour appears only if $\delta\left(\varepsilon-\alpha \sigma^{2}\right)^{2} \neq 0$. It means that in the deterministic case $\sigma^{2}=0$ the nonlinearity is caused by $\delta \neq 0$, whereas the stochastic contribution leading to renormalization of the control parameter $\varepsilon$ is able to promote essential contribution to the above effect. Indeed, at large difference between $\alpha \sigma^{2}$ and $|\varepsilon|$ at fixed $\alpha$, the nonlinear effect becomes well pronounced.

To relate the stochastic approach to the deterministic one, let us rewrite the dimensionless dispersion relation in the form

$$
\omega(k)=-\frac{i}{2 \delta} \pm \sqrt{\frac{k^{2}\left(\tilde{f}_{x x}^{\prime \prime}+r_{c}^{2} k^{2}\right)}{\delta}-\frac{1}{4 \delta}},
$$

where the notation $\tilde{f}_{x x}^{\prime \prime}=f_{x x}^{\prime \prime}+\alpha \sigma^{2}$ is used, $\left.f_{x x}^{\prime \prime}\right|_{x=0}=-\varepsilon$. From this, it follows that the internal noise influence leads to a change in the barrier height for the effective free energy $\tilde{f}$ due to $\left.\tilde{f}_{x x}^{\prime \prime}\right|_{x=0}=-\varepsilon+\alpha \sigma^{2}$. Moving back to dimensional variables, let us interpret our results for the stochastic case. Here, instead of originally exploited correlation and diffusion lengths, $l_{C}=r_{c} / \sqrt{-f_{x x}^{\prime \prime}}$ and $l_{D}=\sqrt{-M_{0} f_{x x}^{\prime \prime} \tau_{D}}$, respectively, it is 


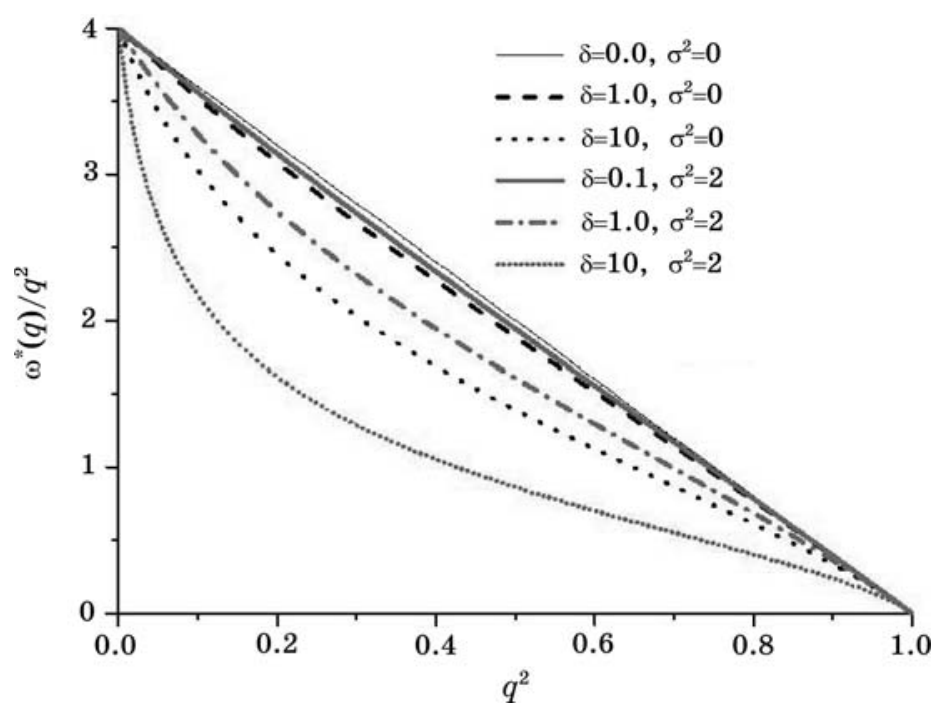

Fig. 23. Comparison of the function $\omega^{*}(q) / q^{2}$ for hyperbolic (modified Cahn-Hilliard) model for deterministic and stochastic cases at different values of the ratio $\delta=\tau_{D} / \tau_{x}$ and different noise intensities $\sigma^{2}$. Other parameters are as follows: $\varepsilon=0.5, D=1, \alpha=0.8$.

convenient to use the effective lengths, $\tilde{l}_{C}=r_{c} / \sqrt{-\tilde{f}_{x x}^{\prime \prime}}$ and $\tilde{l}_{D}=\sqrt{-M_{0} \tilde{f}_{x x}^{\prime \prime} \tau_{D}}$. In such a case, we arrive at formula for the amplification rate given by Eq. (66). The difference in deterministic and stochastic cases lies only in the renormalization of the barrier height for the effective free energy $\tilde{f}$. An increase in the intensity $\sigma^{2}$ of multiplicative fluctuations determined by the field dependent mobility decreases the length $\tilde{l}_{D} \propto \sqrt{\varepsilon-\alpha \sigma^{2}}$ and increases the scale $\tilde{l}_{C} \propto 1 / \sqrt{\varepsilon-\alpha \sigma^{2}}$. The local nonequilibrium effect at small $\sigma^{2}$ is enhanced by the diffusion flux relaxation that is in good correspondence with results obtained for the deterministic case analysis.

\subsubsection{The Effective Fokker-Planck Equation for the Hyperbolic Model}

To describe the system states, we need to know the distribution function of the field $x$. In order to get it, one should obtain the corresponding Fokker-Planck equation. According to the standard procedure, we represent our system in a discrete $d$-dimensional space with $N^{d}$ cells with the mesh size $\ell$. Then, following Eq. (171), the system dynamics will be described by a set of equations for every cell of the space: 


$$
\delta \frac{d^{2} x_{i}}{d t^{2}}+\frac{d x_{i}}{d t}=\nabla_{i j}^{L}\left(M_{j} \nabla_{j l}^{R} \frac{\partial F}{\partial x_{l}}+g_{j} \zeta_{j}\right) .
$$

Next, let us introduce a new variable, $p_{i}$, playing a role of an effective momentum, $p_{i}=\delta \dot{x}_{i}$. Then, by definition, the probability density function is given by the averaging of the density function $\rho(x, p, t)$ over noise: $\mathcal{P}(x, p, t)=\langle\rho(x, p, t)\rangle$. To construct an equation for the macroscopic density function $P$, we exploit the conventional device to proceed from the continuity equation:

$$
\frac{\partial \bar{\rho}}{\partial t}+\sum_{i}\left[\frac{\partial}{\partial x_{i}} \dot{x}_{i}+\frac{\partial}{\partial p_{i}} \dot{p}_{i}\right] \bar{\rho}=0 .
$$

Inserting the momentum definition, we obtain

$$
\frac{\partial \bar{\rho}}{\partial t}=(e+\mathcal{N} \zeta) \rho
$$

where the operators $\mathcal{E}=\sum_{i} e_{i}$ and $\mathcal{N}=\sum_{i} \mathcal{N}_{i}$ are defined as follows

$$
\begin{gathered}
\ell_{i} \equiv-\frac{p_{i}}{\delta} \frac{\partial}{\partial x_{i}}-\frac{\partial}{\partial p_{i}}\left(\nabla_{i j}^{L} M_{j} \nabla_{j l}^{R} \frac{\partial F}{\partial x_{l}}-\frac{1}{\delta} p_{i}\right), \\
\mathcal{N}_{i} \equiv-\nabla_{i j}^{L} g_{j} \frac{\partial}{\partial p_{i}} .
\end{gathered}
$$

Within the interaction representation, the density function reads $\wp=\exp (-\ell t) \rho$ that allows to rewrite Eq. (180) as

$$
\begin{aligned}
\frac{\partial}{\partial t} \wp & =\mathcal{R} \wp, \\
\mathcal{R}=\sum_{i} \mathcal{R}\left(x_{i}, p_{i}, t\right) & \equiv \sum_{i} \zeta_{i}\left[e^{-\ell t} \mathcal{N}_{i} e^{\ell t}\right] .
\end{aligned}
$$

The well-known cumulant expansion method serves as standard and effective device to solve such a type stochastic equation [92]. Neglecting terms of the order $O\left(R^{3}\right)$, we get the kinetic equation for the averaged quantity $\langle\wp(t)\rangle$ in the form

$$
\frac{\partial}{\partial t}\langle\wp\rangle(t)=\left[\int_{0}^{t}\left\langle\mathcal{R}(t) \mathcal{R}\left(t^{\prime}\right)\right\rangle d t^{\prime}\right]\langle\wp\rangle(t) .
$$

Within the original representation, the equation for the probability density reads 


$$
\frac{\partial}{\partial t} P(t)=\left\{\ell+\int_{0}^{t} C(\tau)\left[\mathcal{N}\left(e^{\ell \tau} \mathcal{N} e^{-\ell \tau}\right)\right] d \tau\right\} P(t) .
$$

Due to the physical time much larger than a correlation scale $(t>\tau)$, one can replace the upper limit of the integration by $\infty$.

To proceed this one, we use the procedure proposed in Ref. [69, 70] to obtain the effective Fokker-Planck equation for the hyperbolic stochastic model. Expanding exponents, we arrive at the equation

$$
(\partial / \partial t-e) P=C P
$$

where the operator

$$
e \equiv e_{(1)}+e_{(2)}
$$

has the components

$$
\ell_{(1)} \equiv-\sum_{i} \frac{p_{i}}{\delta} \frac{\partial}{\partial x_{i}}+\left(\nabla_{i j}^{L} M_{j} \nabla_{j l}^{R} \frac{\partial F}{\partial x_{l}}\right) \frac{\partial}{\partial p_{i}}, \quad \ell_{(2)} \equiv \frac{1}{\delta} \sum_{i} \frac{\partial}{\partial p_{i}} p_{i} .
$$

The collision operator $C$ is defined as follows:

$$
C=\sum_{n=0}^{\infty} C^{(n)}, C^{(n)}=\mathcal{H}^{(n)}\left(\mathcal{N} \mathcal{E}^{(n)}\right), \ell^{(0)}=\mathcal{N}, \mathcal{N} \equiv-\sum_{i} \nabla_{i j}^{L} g_{j} \frac{\partial}{\partial p_{i}},
$$

where $e^{(n)}$ in the collision operator $C$ is defined through the commutator $e^{(n)}=\left[\ell, \ell^{(n-1)}\right]$; moments of the noise correlation function $C(\tau)$ are defined as follows:

$$
\mathcal{M}^{(n)}=(n !)^{-1} \int_{0}^{\infty} \tau^{n} C(\tau) d \tau
$$

Substituting all definitions into commutators, one can calculate the collision operator in the form:

$$
\begin{gathered}
C=-\sum_{i}\left(\mathcal{M}^{(0)}-\mathcal{M}^{(1)}\right) \nabla_{i j}^{L} g_{j} \nabla_{j l}^{R} g_{l} \frac{\partial^{2}}{\partial p_{i}^{2}}+ \\
+\mathcal{M}^{(1)}\left\{\nabla_{i j}^{L} g_{j} \nabla_{j l}^{R} g_{l} \frac{\partial^{2}}{\partial x_{i} \partial p_{i}}-\nabla_{i j}^{L} g_{j}\left(\frac{\partial \nabla_{j l}^{R} g_{l}}{\partial x_{l}}\right)\left(\frac{\partial}{\partial p_{i}}+p_{i} \frac{\partial^{2}}{\partial p_{i}^{2}}\right)\right\} .
\end{gathered}
$$

Noting that, in further consideration, we are interesting in behaviour of the distribution $\mathcal{P}\left(\left\{x_{i}\right\}, t\right)$, not the total one, $P\left(\left\{x_{i}\right\},\left\{p_{i}\right\}, t\right)$. The reduced distribution can be obtained according to the moments

$$
P^{(n)}\left(\left\{x_{i}\right\}, t\right) \equiv \int \mathcal{P}\left(\left\{x_{i}\right\},\left\{p_{i}\right\}, t\right) \prod_{i}\left[p_{i}^{n} \mathrm{~d} p_{i}\right],
$$


where the integration is provided over a set $\left\{p_{i}\right\}$. Then, performing corresponding manipulations with Eq. (187), we arrive at the recursive relations for the moments $P^{(n)}\left(\left\{x_{i}\right\}, t\right)$, where $P \equiv P^{(0)}\left(\left\{x_{i}\right\}, t\right)$, the first moment $P^{(1)}\left(\left\{x_{i}\right\}, t\right)$ can be considered as a flux $\mathcal{J}$ of the probability density, i.e. $P^{(1)}=\mathcal{J}$.

Indeed, taking the zeroth moment of momentum, $p$, over Eq. (187), we obtain the expected continuity equation

$$
\partial_{t} P=-\frac{1}{\delta} \sum_{i} \frac{\partial}{\partial x_{i}} \mathcal{J}
$$

The first-moments' calculation leads to

$$
\begin{gathered}
\partial_{t} \mathcal{J}=-\frac{1}{\delta} \mathcal{J}+\sum_{i}\left[\left(\nabla_{i j}^{L} M_{j} \nabla_{j l}^{R} \frac{\partial F}{\partial x_{l}}\right) P+\right. \\
\left.+\mathcal{M}^{(1)}\left\{\nabla_{i j}^{L} g_{j} \nabla_{j l}^{R} g_{l} \frac{\partial P}{\partial x_{i}}+\nabla_{i j}^{L} g_{j}\left(\frac{\partial \nabla_{j l}^{R} g_{l}}{\partial x_{l}}\right) P\right\}-\frac{1}{\delta} \frac{\partial P^{(2)}}{\partial x_{i}}\right] .
\end{gathered}
$$

For the second moment, we obtain

$$
\frac{1}{\delta} \frac{\partial P^{(2)}}{\partial x_{i}}=\left(\mathcal{M}^{(1)}-\mathcal{M}^{(0)}\right) \nabla_{i j}^{L} g_{j} \nabla_{j l}^{R} g_{l} P .
$$

As a result, the evolution equation for the flux $\mathcal{J}$ is of the form

$$
\begin{aligned}
\partial_{t} \mathcal{J}=-\frac{1}{\delta} \mathcal{J}+\sum_{i} & {\left[\left(\nabla_{i j}^{L} M_{j} \nabla_{j l}^{R} \frac{\partial F}{\partial x_{l}}-\mathcal{M}^{(1)} \nabla_{i j}^{L} g_{j}\left(\frac{\partial \nabla_{j l}^{R} g_{l}}{\partial x_{l}}\right)\right) P+\right.} \\
& \left.+\mathcal{M}^{(0)} \frac{\partial}{\partial x_{i}} \nabla_{i j}^{L} g_{j} \nabla_{j l}^{R} g_{l} P\right] .
\end{aligned}
$$

Therefore, we arrive at the set of two differential equations, Eq. (194) and Eq. (197). Eliminating the flux $\mathcal{J}$, we, finally, get the Fokker-Planck equation for the hyperbolic stochastic model in the form

$$
\begin{gathered}
\delta \frac{\partial^{2} P}{\partial t^{2}}+\frac{\partial P}{\partial t}=-\sum_{i} \frac{\partial}{\partial x_{i}}\left[\nabla_{i j}^{L} M_{j} \nabla_{j l}^{R} \frac{\partial F}{\partial x_{l}}-M^{(1)} \nabla_{i j}^{L} g_{j}\left(\frac{\partial \nabla_{j l}^{R} g_{l}}{\partial x_{j}}\right)\right] P- \\
-\mathcal{M}^{(0)} \sum_{i j} \frac{\partial^{2}}{\partial x_{i} x_{j}} \nabla_{i j}^{L} g_{j} \nabla_{j l}^{R} g_{l} P .
\end{gathered}
$$

In the continuum space, the obtained effective Fokker-Planck equation for the probability density functional is as follows: 


$$
\begin{aligned}
& \delta \partial_{t t}^{2} P+\partial_{t} P=-\int d \mathbf{r} \frac{\delta}{\delta x(\mathbf{r})}\left[\nabla M \nabla \frac{\delta \mathscr{F}}{\delta x(\mathbf{r})}-\frac{M^{(1)}}{2} \nabla\left(\nabla \frac{\delta M}{\delta x(\mathbf{r})}\right)\right] P- \\
&-M^{(0)} \int d \mathbf{r} \frac{\delta^{2}}{\delta x^{2}(\mathbf{r})} \nabla(\nabla M) P .
\end{aligned}
$$

The stationary probability density can be obtained explicitly under no flux conditions. Indeed, here, we arrive at the result

$$
P_{s}[x] \propto \exp \left\{-\frac{1}{M^{(0)}}\left(F[x]+\left(M^{(0)}-\mathcal{M}^{(1)}\right) \int d \mathbf{r} \ln M(x)\right)\right\} .
$$

This is similar to that presented in Eq. (100). The main difference is that in the distribution (200) we have a contribution related to the first moment of the noise correlation function with respect to the time. It means that the stochastic source appeared in the hyperbolic model should have smeared temporal correlation function. In other words, its frequency spectrum should have some cut-off frequency and the noise should be considered as a coloured noise in time. It looks absolutely natural, because as it was shown in previous subsection the stochastic hyperbolic transport should be characterized with fixed correlation time, $\tau_{\zeta}$, which may be small comparing to the relaxation time for the concentration field. Taking the temporal correlation function in the exponential form related to the Ornstein-Uhlenbeck process $\zeta$, $C\left(t-t^{\prime}\right)=\tau_{\zeta}^{-1} \exp \left(-\left|t-t^{\prime}\right| / \tau_{\zeta}\right)$, one can find $\mathcal{M}^{(0)}=\sigma^{2}, M^{(1)}=\tau_{\zeta} \sigma^{2}$. It is seen that the temporal correlation radius $\tau_{\zeta}$ makes the renormalization of the critical values for the noise intensity, whereas main results obtained for the stochastic parabolic model remain the same.

Making use the fluctuation dissipation relation one can relate the correlation scale with the relaxation time for the flux, $\tau_{\zeta} ; \tau_{D}$. It follows that, in the white noise limit characterized by $\tau_{\zeta} \rightarrow 0$, one gets the parabolic model for the spinodal decomposition, whereas one arrives at the hyperbolic model at fixed but small $\tau_{\zeta}$. This conclusion is in a good correspondence with the linear stability analysis, where it was shown that in order to get the nonlinear dependence of the amplification rate it is necessary to consider two modes: concentration field and flux.

\section{CONCLUSIONS}

A model for kinetics of fast spinodal decomposition in a binary system free from imperfections and with the molar volume independent of concentration is developed. The model takes into account a finiteness of the diffusion speed $V_{D}$ and assumes that the spinodal decomposition may proceed with the rate of the order of $V_{D}$. Such an approach leads to the description with independent variables of the concentration and 
atomic diffusion flux having different relaxation times to their own local equilibrium values. It leads to a modified Cahn-Hilliard equation, describing the spinodal decomposition for both diffusion and wave propagation of atoms (components of the binary system). In such a case, the equation describes kinetics of fast separation the rate of which compatible with the diffusion speed $V_{D}$.

To describe fast decomposition, the free energy density $f(c, \nabla c, \mathbf{J})=f_{\mathrm{e}}+f_{\mathrm{ne}}$ includes standard local equilibrium contribution $f_{\mathrm{e}}=f(c, \nabla c)$ and purely non-equilibrium contribution $f_{\text {ne }}$ proportional to the square of the atomic flux, $\mathbf{J} \cdot \mathbf{J}$. The use of $f(c, \nabla c, \mathbf{J})$ in such a form has a statistical basis: there are many particles within every local volume, and it includes a reduction of the available phase space for each particle. It is shown that the fast spinodal decomposition is described by a hyperbolic type of differential equation. This description is true if the time scale of the process of phase separation has the order of the relaxation time $\tau_{D}$. It occurs for the case of the fast frequency and short wave-lengths which cannot be neglected in the description of evolution from an unstable state to a new metastable state in spinodally-decomposing system and, generally, for fast moving interfaces. Despite classic Cahn-Hilliard scenario described by parabolicdiffusion equation predicts much more diffuse boundaries, the hyperbolic scenario exhibits evolution with sharper boundaries between two separating phases. It occurs due to description of hyperbolic evolution by the equation with a finite diffusion speed and description of the parabolic evolution by the equation with an infinite diffusion speed.

The provided analysis leads to the obtaining the phase and group atomic speeds. The real values for speeds define the finite propagation of a single harmonic (for the phase speed) and a packet of harmonics (for the group speed). The hyperbolic model is able to give prediction for scenario from very earliest up to latest of spinodal decomposition. We considered the fluctuations of the solute density and the solute diffusion flux at the equilibrium steady state. The power spectra of a solute number density and a solute diffusion flux have been reviewed. The latter has a non-vanishing relaxation time leading to a hyperbolic transport equation for the evolution of the density. Several interpretations of the stochastic source related to fast variables eliminated from the description have been examined. Particularly, the phase separation scenario of the system with internal multiplicative noise related to the field dependent mobility for parabolic (Cahn-Hilliard) and hyperbolic models is analyzed. Analysis was performed for early and late stages of the evolution analytically and by computer simulations. The stationary case is considered with the help of the mean field approach.

For the system undergoing spinodal decomposition with the field dependent mobility, we have derived the Fokker-Planck equation. It was found that its stationary solution can be written in exact form. 
Our theoretical approach shows that we dealt with the entropy driven phase transitions mechanism. As shown, the field-dependent mobility leads to delays in dynamics at early stages and, therefore, leads to delays in domain growth law at late stages. Stationary values of the order parameter reduced to the second moment of the stochastic field depend on the parameter that governs the functional dependence of the mobility: with an increase in such parameter, the order is suppressed. Considering stationary states, we extend the mean field approach to the systems with the field-dependent mobility. It was found that an increase in the parameter that governs the functional dependence of the mobility, the critical points for the phase transitions are shifted. The system can undergo a re-entrant phase transitions when the mean field becomes nontrivial inside the fixed domain of the noise intensity. The strong coupling regime shows that a position of the critical point depends on the constant governing the field-dependent mobility.

Our results can be applied to investigations of polymer mixtures where relaxation flows are driven by field-dependent coefficients, phase separation in binary alloys, and microstructure phase transitions.

\section{ACKNOWLEDGMENTS}

We thank David Jou and Alexander Olemskoi for fruitful discussions and useful exchanges. Dmitrii Kharchenko acknowledges financial support from the Fundamental Research State Fund of Ukraine (No. GP/F26/0010). Peter Galenko acknowledges financial support from the German Research Foundation (DFG) under the Project No. HE 160/19 and DLR Agency under contract 50WM0736. Vladimir Lebedev acknowledges financial support from the Russian Foundation of Basic Research (RFBR) under the Project No. 08-02-91957.

\section{REFERENCES}

1. H. Jinnai, T. Hashimoto, D. Lee, and S.-H. Chen, Macromolecules, 30: 130 (1997).

2. F. Mallamace, N. Micali, and S. Trusso, J. Phys.: Condens. Matter, 8: A81 (1996).

3. N. F. Bunkin, A. V. Lobeev, and G. A. Lyakhov, Usp. Fiz. Nauk, 167: 1070 (1997) (Physics-Uspekhi, 40: 1090 (1997)); Yu. I. Ustinovshchikov and B. E. Pushkarev, Usp. Fiz. Nauk, 176: 611 (2006) (Physics-Uspekhi, 49: 593 (2006)).

4. D. Beysens, Materials Sciences in Space (Eds. B. Feuerbacher, H. Hamacher, and R. J. Naumann) (Berlin: Springer: 1986), p. 191.

5. J. Mainville, Y. S. Yang, K. R. Elder, M. Sutton, K. F. Ludwig, and G. B. Sephenson, Phys. Rev. Lett., 78: 258 (1997). 
6. Yu. I. Ustinovshchikov and B. E. Pushkarev, Usp. Fiz. Nauk, 176: 611 (2006) (Physics-Uspekhi, 49: 593 (2006)).

7. J. S. Langer and M. Baron, Annals of Physics, 78: 421 (1973); J. P. Donley and J. S. Langer, Phys. Rev. Lett., 71: 1573 (1993).

8. $\quad$ G. F. Mazenko, Phys. Rev. B, 43: 5747 (1991).

9. K. Binder, C. Billotet, and P. Mirold, Zeitschrift für Physik B-Condensed Matter, 30: 183 (1978); K. Binder, J. of Chemical Physics, 79: 6387 (1983).

10. K. Binder and P. Fratzl, Phase Transformations in Materials (Ed. G. Kostorz) (Weinheim: Wiley: 2001), p. 409.

11. V. L. Ginzburg and L. D. Landau, JETP, 20: 1064 (1950).

12. J. W. Cahn and J. E. Hilliard, J. Chem. Phys., 28: 258 (1958).

13. J. W. Cahn, Acta Metall., 9: 795 (1961).

14. J. W. Cahn, Trans. Metall. Soc. AIME, 242: 166 (1968).

15. V. P. Skripov and A. V. Skripov, Usp. Fiz. Nauk, 128: 193 (1979) (Sov. Phys. Usp., 22: 389 (1979)).

16. S. Bastea and J. L. Lebovitz, Phys. Rev. E, 52: 3821, (1995).

17. B. Liu, H. Zhang, and Y. Yang, J. Chem. Phys., 113: 719, (2000).

18. K. Binder, H. L. Frish, and J. Jäckle, J. Chem Phys., 85: 1505 (1986).

19. P. Galenko, Phys. Lett. A, 287: 190 (2001).

20. P. Galenko and D. Jou, Phys. Rev. E, 71: 046125 (2005).

21. D. Jou, J. Casas-Vazquez, and G. Lebon, Extended Irreversible Thermodynamics (Berlin: Springer: 2001).

22. M. Grasselli and V. Pata, J. Evolution Equations, 4: 27 (2004).

23. S. Gatti, M. Grasselli, A. Miranville, and V. Pata, J. Math. Anal. Appl., 312: 230 (2005).

24. M. Grasselli, G. Schimperna, and S. Zelik, arXiv:0804.0988v1 [math.AP].

25. A. Segatti, Math. Models Methods Appl. Sci., 17, No. 3: 411 (2007).

26. P. Galenko and S. Sobolev, Phys. Rev. E, 55: 343 (1997).

27. $\quad$ P. K. Galenko and D. A. Danilov, Phys. Lett. A, 235: 271 (1997).

28. L. D. Landau and E. M. Lifshitz, Fluid Mechanics (Addison Wesley: 1958).

29. D. Jou and J. M. Rubi, Phys. Lett. A, 72: 78 (1979).

30. D. Jou and C. Perez-Garsia, Physica A, 104: 3320 (1980).

31. D. Jou, J. E. Llebot, and J. Casas-Vazquez, Physica A, 109: 208 (1981).

32. H. Kleinert and S. V. Shabanov, Phys. Lett. A, 235: 105 (1997).

33. M. Ibanes, J. Garcia-Ojalvo, R. Toral, and J. M. Sancho, Lecture Notes in Physics (Berlin: Springer-Verlag: 2000), vol. 557, p. 247.

34. J. M. Sancho, J. Garcia-Ojalvo, and H. Guo, Physica D, 113: 331 (1998).

35. $\quad$ C. Van der Broeck, Phys. Rev. Lett., 73: 3395 (1994).

36. C. Van der Broeck, J. M. R. Parrondo, R. Toral, and R. Kawai, Phys. Rev. $E$, 55: 4084 (1997).

37. M. Ibanes, J. Garcia-Ojalvo, R. Toral, and J. M. Sancho, Phys. Rev. E, 60: 3597 (1999).

38. J. Buceta, M. Ibanes, J. M. Sancho, and K. Lindenberg, arXiv:condmat/0211181.

39. P. K. Galenko and M. D. Krivilyov, Modelling Simul. Mater. Sci. Eng., 8: 67 (2000).

40. D. Jou and P. Galenko, Physica A, 399: 149 (2006).

41. P. Galenko, Phys. Rev. B, 65: 144103 (2002).

42. J. P. Boon and S. Yip, Molecular Hydrodynamics (New York: McGraw Hill: 
1970).

43. H. B. Callen, Thermodynamics (New York: Wiley: 1960).

44. R. Luzzi, A. R. Vasconcellos, J. Casas-Vazquez, and D. Jou, Physica A, 248: 111 (1998).

45. P. Galenko and D. Jou, Proceedings of Joint European Thermodynamics Conference IX (Eds. B. Guy and D. Tondeur) (Saint-Etienne: Ecole Nationale Superieure des Mines: 2007), p. 75.

46. J. Casas-Vazquez and D. Jou, Rep. Prog. Phys., 66: 1937 (2003).

47. P. Galenko and V. Lebedev, Phil. Mag. Lett., 87: 821 (2007).

48. P. Galenko and V. Lebedev, Phys. Lett. A, 372: 985 (2008).

49. P. Galenko and D. Jou, Physica A, 388: 3113 (2009).

50. A. Miranville and S. Zelik, Math. Methods Appl. Sci., 28: 709 (2005).

51. C. Gal, Dyn. Partial Differ. Equ., 5: 39 (2008).

52. P. Galenko and V. Lebedev, The International Journal of Thermodynamics, 11: 21 (2008).

53. P. Galenko, Phys. Rev. B, 65: 144103 (2002).

54. M. Hillert, Acta Metall., 9: 525 (1961).

55. P. Galenko and V. Lebedev, Pis'ma Zh. Eksp. Teor. Phys., 86: 525 (1990) (JETP Lett., 86: 458 (2007)).

56. N. S. Andreev, G. G. Boiko, and N. A. Bokov, J. Non-Cryst. Solids, 5: 41 (1970).

57. N. S. Andreev and E. A. Porai-Koshits, Discuss. Faraday Soc., 50: 135 (1970).

58. E. P. Feldman and L. I. Stefanovich, Zh. Eksp. Teor. Fiz., 98: 1695 (1990) (Sov. Phys. JETP, 71: 951 (1990)).

59. H. E. Cook, J. Chem. Solids, 30: 2427 (1969); H. E. Cook, Acta. Metall., 18: 297 (1970).

60. W. Horsthemke and R. Lefever, Noise-Induced Transitions (Berlin: Springer-Verlag: 1984).

61. H. Risken, The Fokker-Planck Equation (Berlin: Springer-Verlag: 1989).

62. C. W. Gradiner, Handbook of Stochastic Methods (Berlin: Springer-Verlag: 1985).

63. A. I. Olemskoi and D. O. Kharchenko, Physica A, 293: 178 (2001).

64. D. O. Kharchenko and S. V. Kohan, Eur. Phys. J. B, 29: 97 (2002).

65. K. Wiesenfeld and F. Moss, Nature, 373: 33 (1995).

66. L. Gammaitoni, P. Hänggi, P. Jung, and F. Marchesoni, Rev. Mod. Phys., 70: 223 (1998).

67. J. Garcia-Ojalvo and J. M. Sancho, Noise in Spatially Extended Systems (New York: Springer-Verlag: 1999).

68. D. O. Kharchenko, Physica A, 308: 113 (2002).

69. D. O. Kharchenko and I. A. Knyaz', Eur. Phys. J. B, 32: 375 (2003).

70. A. I. Olemskoi, D. O. Kharchenko, and I. A. Knyaz', Phys. Rev. E, 71: 041101 (2005).

71. J. M. R. Parrondo, C. Van der Broeck, J. Buceta, and F. J. de la Rubia, Physica A, 224: 153 (1996).

72. F. Sagués, J. M. Sancho, and J. Garcia-Ojalvo, Rev. Mod. Phys., 79: 829 (2007).

73. B. Linder, J. Garcia-Ojalvo, A. Neiman, and L. Shimansky-Geier, Phys. Rep., 392: 321 (2004). 
74. Y. Tu, G. Grinstein, and M. A. Munoz, Phys. Rev. Lett., 78: 224 (1997).

75. W. Genovese, M. A. Munoz, and J. M. Sancho, Phys. Rev. E, 57: R2495 (1998).

76. M. Ibanes, J. Garcia-Ojalvo, R. Toral, and J. M. Sancho, Phys. Rev. Lett., 87: 020601 (2001).

77. B. von Haeften, G. Izús, S. Mangioni, A. D. Sánchez, and H. S. Wio, Phys. Rev. E, 69: 021107 (2004).

78. D. O. Kharchenko, Usp. Fiz. Met., 6: 1 (2005).

79. E. A. Novikov, Sov. Phys. JETP, 20: 1290 (1965).

80. J. Buceta and K. Lindenberg, Phys. Rev. E, 69: 011102 (2004).

81. D. O. Kharchenko and A. V. Dvornichenko, Eur. Phys. J. B, 61: 95 (2008).

82. D. O. Kharchenko and A. V. Dvornichenko, Physica A, 387: 5342 (2008).

83. S. van Gemmert, G. T. Barkema, and S. Puri, arXiv:cond-mat/0501196.

84. J. Garcia-Ojalvo, A. M. Lacasta, J. M. Sancho, and R. Toral, Europhys. Lett., 42: 125 (1998).

85. L. Ramirez-Piscina, J. M. Sancho, and A. Hernandez-Machado, Phys. Rev. B, 48: 125 (1993).

86. I. M. Lifshitz and V. V. Slyozov, J. Phys. Chem. Solids, 19: 35 (1961).

87. C. L. Emmott and A. J. Bray, Phys. Rev. E, 59: 213 (1999).

88. J. M. Sancho, A. Hernandez-Machado, L. Ramirez-Piscina, and A. M. Lacasta, Acta Phys. Polonica B, 24: 733 (1993).

89. J. D. Gunton, M. San Miguel, and P. S. Sahni, Phase Transtions and Critical Phenomena (New York: Academic Press: 1983), vol. 8.

90. D. O. Kharchenko, A. V. Dvornichenko, and I. A. Lysenko, Ukr. Jour. of Phys., 53, No. 9: 917 (2008).

91. S. De Groot and P. Mazur, Non-Equilibrium Thermodynamics (Amsterdam: 1962).

92. N. G. Van Kampen, Stochastic Processes in Physics and Chemistry (Amsterdam: 1992). 